\title{
Spreader-Bar Radiation Detection System Enhancements: A Modeling and Simulation Study
}

Revision 0

JH Ely

ED Ashbaker

MT Batdorf

JE Baciak

WK Hensley

KD Jarman

SM Robinson

GA Sandness

JE Schweppe

October 2012 


\title{
DISCLAIMER
}

This report was prepared as an account of work sponsored by an agency of the United States Government. Neither the United States Government nor any agency thereof, nor Battelle Memorial Institute, nor any of their employees, makes any warranty, express or implied, or assumes any legal liability or responsibility for the accuracy, completeness, or usefulness of any information, apparatus, product, or process disclosed, or represents that its use would not infringe privately owned rights. Reference herein to any specific commercial product, process, or service by trade name, trademark, manufacturer, or otherwise does not necessarily constitute or imply its endorsement, recommendation, or favoring by the United States Government or any agency thereof, or Battelle Memorial Institute. The views and opinions of authors expressed herein do not necessarily state or reflect those of the United States Government or any agency thereof.

\author{
PACIFIC NORTHWEST NATIONAL LABORATORY \\ operated by \\ BATTELLE \\ for the \\ UNITED STATES DEPARTMENT OF ENERGY \\ under Contract DE-AC05-76RL01830
}

Printed in the United States of America
Available to DOE and DOE contractors from the Office of Scientific and Technical Information,
P.O. Box 62, Oak Ridge, TN 37831-0062;
ph: (865) 576-8401
fax: $(865)$ 576-5728
email: reports@adonis.osti.gov

\begin{abstract}
Available to the public from the National Technical Information Service, U.S. Department of Commerce, 5285 Port Royal Rd., Springfield, VA 22161 ph: (800) 553-6847 fax: $(703) 605-6900$ email: orders@ntis.fedworld.gov online ordering: http://www.ntis.gov/ordering.htm
\end{abstract}

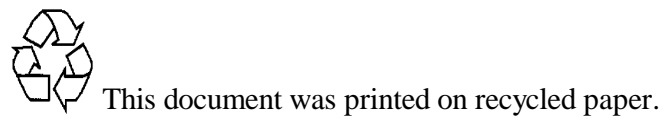




\section{Spreader-Bar Radiation Detector System Enhancements: A Modeling and Simulation Study}

JH Ely

ED Ashbaker

MT Batdorf

JE Baciak

WK Hensley

KD Jarman

SM Robinson

GA Sandness

JE Schweppe

October 2012

Prepared for the U.S. Department of Energy

under U.S. Department of Energy Contract DE-AC05-76RL01830

Pacific Northwest National Laboratory

Richland, Washington 99352 


\section{Executive Summary}

An investigation of radiation detectors on crane-mounted platforms was performed to understand the limitations and optimal capability of these types of systems. Crane-mounted systems are primarily focused on crane-mounted radiation detector systems, where the detectors are mounted on a spreader-bar platform. Spreader bars are attached with cables to a crane at seaports to load and unload containers from cargo ships. The bars can spread from 20 feet to 53 feet using hydraulics, allowing a single bar to move a variety of container lengths. Spreader-bars are also used on straddle carriers, which move containers around the laydown yard in the port.

Spreader bar radiation detection (SBRD) systems are commercially available from at least two companies. These systems employ a set of spectroscopic sensors placed on the underside of a gantry crane and held near the top of a cargo container for detection. These systems have been previously tested and some issues and limitations observed. Radiation detectors mounted on spreader-bars are single sided systems (observing cargo only from the top) and it is therefore challenging to obtain the same sensitivity as double-sided systems (for example, portal monitors) especially when considering the possible amounts of intervening cargo that could be present. The approximately exponential attenuation of gamma rays in intervening dense cargo presents an inherent limitation to detection. However, SBRD systems hold detectors closer to the container, have a longer dwell time, and may have lower background (over the ship); all of these aspects help mitigate the single-sided issue. In addition, there are operational considerations that make spreader-bar radiation systems attractive. These include scanning for radiation during other operations (loading, unloading or container movement) which minimizes additional time for scanning, incorporating the radiation detection into existing equipment to minimize initial cost, and in the case of ship unloading, scanning the containers before reaching the laydown yard.

This study is focused on researching optimized configurations of SBRD systems, including number of detectors, locations, and best use of detector data, to determine a reasonable limit on the capability of these types of systems. The results will help support decisions on where and how these systems might be effectively deployed, and could be used for future specification development for SBRD acquisition.

The next phase of this research and investigation into SBRD systems and quantification of the possible improved capability with an enhanced SBRD is the focus of this report. Supporting this investigation, detailed models of an enhanced SBRD system were created using a 3D radiation transport code, and models were run to simulate the data from sources and background for a realistic cargo-loading port environment. The enhanced SBRD had increased detector material (numbers of detectors) compared to the commercial systems, with a distribution providing more uniform coverage. Measurements using small industrial sources were made with an enhanced SBRD system developed by Sandia National Laboratory to support model validation to establish credibility for the simulations of these threat and background scenarios.

A significant simulation effort was undertaken to simulate sources of interest within a 40-foot cargo container. In order to estimate the performance of a generalized SBRD system in scenarios including background and cargo, a highly enriched uranium (HEU) test source (with $3 \times 10^{-8}{ }^{232} \mathrm{U}$ by weight) within a wood cargo model was simulated for a 40 -foot cargo container. This source was placed at a variety of locations, and the flux from the source was multiplied as necessary to create estimated minimal detectable quantities (MDQ) figures for various quantities of HEU. Simulated data sets were produced corresponding to a complete container offloading scenario for a large number of statistical realizations. 
Significant work was performed on both the algorithm development and the container cargo distributions entering the US, and the result of this modeling, validation, and simulation effort was a set of estimates of the performance of a variety of radiation detection algorithms in this cargo-screening environment. In the case of algorithms, a variety of contemporary radiation detection and identification approaches were employed, and the optimal approaches to selecting time and location subsets of detector data were explored. In all cases, algorithm performance was compared, and the best approaches were suggested along with the best expected performance from the enhanced SBRD. In general, template-matching schemes employing a limited set of regression parameters tend to provide best results.

The improvements of an enhanced SBRD with increased detector material and improved algorithms result in an effective MDQ of $10 \mathrm{~kg}$ of HEU in 50 percent of the imported cargo in 40-foot containers, assuming a random source location. This is approximately two times the capability of the estimated performance of the commercial systems. For an MDQ of the $10 \mathrm{~kg}$ HEU source located in the worst location (bottom of the 40-foot container), the volume of cargo scanned drops to $10 \%$ for the enhanced SBRD, again a factor of two or more larger than the commercial systems. Enhancing the SBRD systems by adding more detectors, providing a more uniform coverage, and incorporating more advanced algorithms will result in a significant increase in capability.

Although this investigation provided some indication of the performance capabilities of an enhanced SBRD system, additional work is needed to estimate the capability for other container configurations, such as the 20 and 45 foot containers. Since the 20 -foot container weight limit is similar to the 40 -foot, more dense cargo is typically shipped in 20 -foot containers. This will result in a smaller percentage of cargo scanned for the 20-foot containers (for a particular source quantity) compared to the 40-foot containers. In addition to other configuration, other source material needs to be investigated to understand the capabilities for the entire threat space, where only HEU is considered in this work. 


\section{Acronyms and Abbreviations}

$\begin{array}{ll}\text { CBP } & \text { U.S. Customs and Border Protection } \\ \text { cps } & \text { counts per second } \\ \text { DOE } & \text { U.S. Department of Energy } \\ \text { ED } & \text { energy distance } \\ \text { GC } & \text { gross count } \\ \text { GADRAS } & \text { Gamma Detector Response and Analysis Software } \\ \text { HEU } & \text { Highly enriched uranium } \\ \text { IMCC } & \text { inter modal cargo container } \\ \text { LANL } & \text { Los Alamos National Laboratory } \\ \text { MCNP } & \text { Monte Carlo N-Particle Transport Code } \\ \text { MDA } & \text { minimum detectable activity } \\ \text { MDQ } & \text { minimum detectable quantity } \\ \text { NaI(T) } & \text { thallium-doped sodium iodide } \\ \text { NFAR } & \text { muisance/false alarm rate } \\ \text { NORM } & \text { naturally occurring radioactive material } \\ \text { PIERS } & \text { Port Import Export Reporting Service } \\ \text { PNNL } & \text { Pacific Northwest National Laboratory } \\ \text { POE } & \text { port of entry } \\ \text { PVT } & \text { Polyvinyl toluene } \\ \text { QuID } & \text { Quick ID } \\ \text { RPM } & \text { radiation portal monitor } \\ \text { RPMP } & \text { Radiation Portal Monitor Project } \\ \text { RSP } & \text { radiation sensor panel } \\ \text { SAIC } & \text { Science Applications International Corporation } \\ \text { SBRD } & \text { spreader-bar radiation detection system } \\ \text { SLD } & \text { Second Line of Defense } \\ \text { SNL } & \text { Sandia National Laboratory } \\ \text { SNM } & \text { special nuclear material } \\ \text { SNR } & \text { signal-to-noise ratio } \\ \text { SWF } & \text { twelve-foot equivalent units } \\ \text { TEU } & \end{array}$


Table of Contents

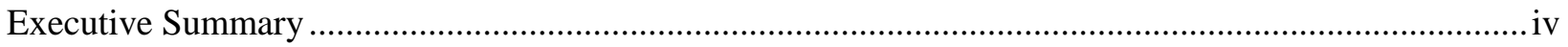

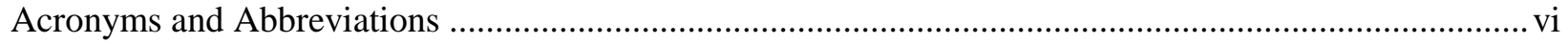

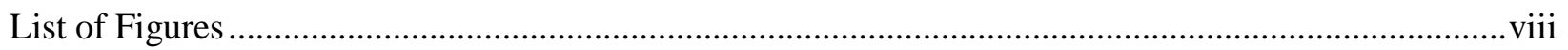

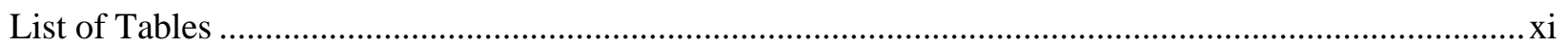

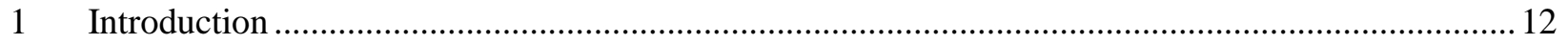

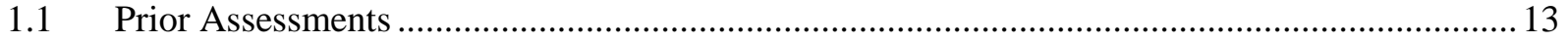

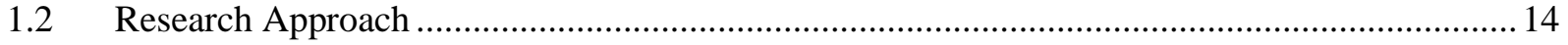

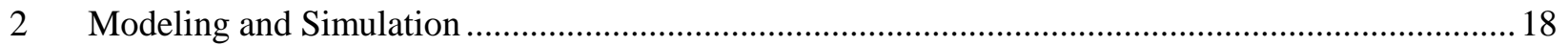

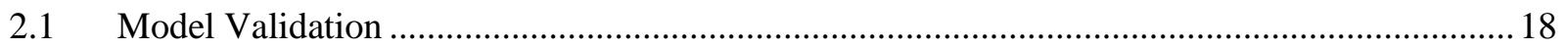

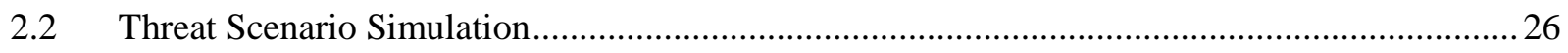

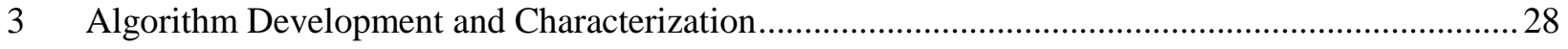

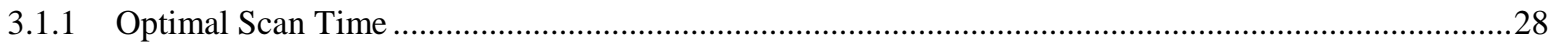

3.1.2 Optimal Detector Subset ..............................................................................................................

3.1.3 Optimal Detector Subset - Iterative Methods ……................................................................................38

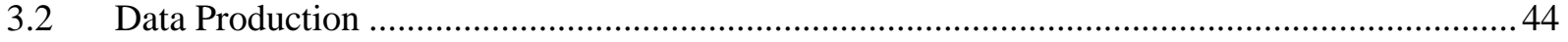

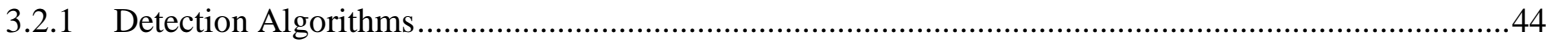

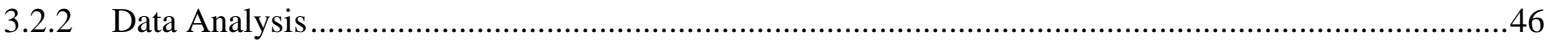

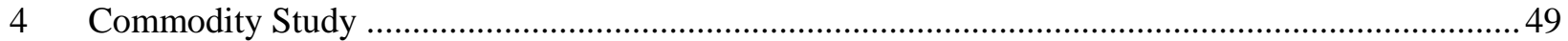

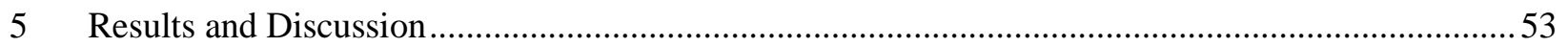

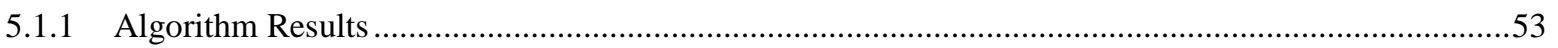

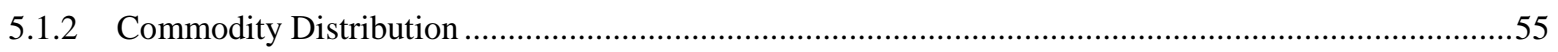

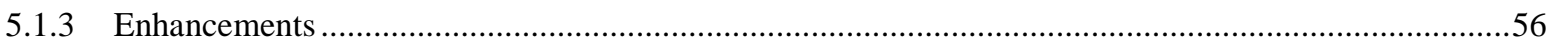

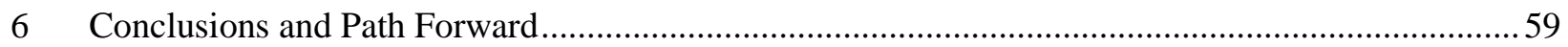

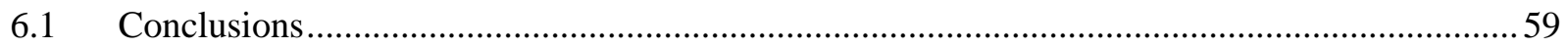

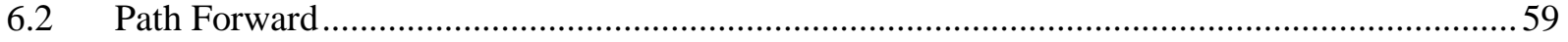

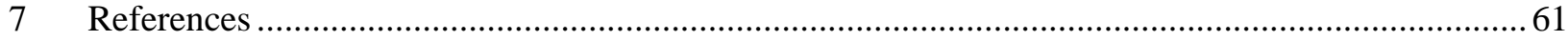

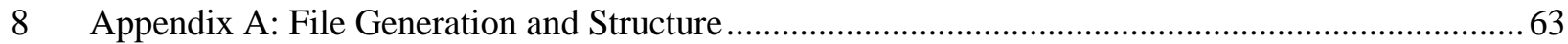

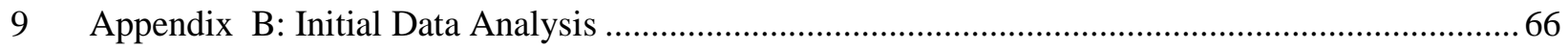




\section{List of Figures}

Figure 1. Picture of a spreader-bar (white bar) above the container, and attached to the crane block used

for loading and unloading ships. Similar spreader-bars are used on straddle carriers.

Figure 2. Total percentage of cargo scanned as a function of the targeted MDQ for the estimated commercial capability (2008) and enhanced SBRD (2010) for a random source location.....

Figure 3. Total percentage of cargo scanned as a function of the targeted MDQ for the estimated commercial capability (2008) and enhanced SBRD (2010) for a source location on the container bottom.

Figure 4. Photograph of the SBRD, before being placed on the 40-foot IMCC.

Figure 5. Relative horizontal detector positions (blue squares) and source positions (red circles)........... 19

Figure 6. Comparison of simulated to measured spectra for source positions 1 through 3 .....................20

Figure 7. Comparison of simulated to measured spectra for source positions 4 through 6 ......................21

Figure 8. Comparison of simulated to measured spectra for source positions 7 through 9 .....................22

Figure 9. Comparison of simulated to measured spectra for source positions 10 through 12 ...................23

Figure 10. Comparison of simulated to measured spectra for source positions 13 through 15 ................24

Figure 11. Plots of the fractional difference between the simulated and measured spectra for the four

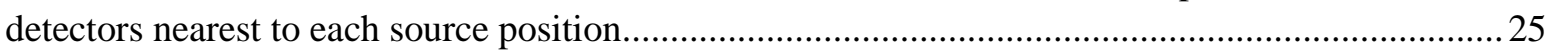

Figure 12. Plot of the fractional difference between the simulated and measured spectra for the four

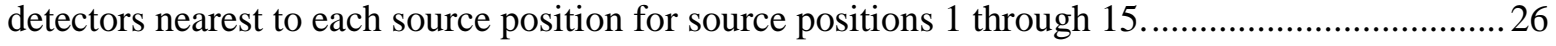

Figure 13. Simulated spectra in the central detector for the asphalt background. .27

Figure 14. Count rate in a single detector as a function of time as the detector is moved back from over the ship to the dock.

Figure 15. Signal-to-noise ratio in a single detector as it is moved back from over the ship to the dock.

The lower, blue curve is calculated for separate, one-second intervals while the upper, red curve is calculated for the entire cumulative time.

Figure 16. Energy distribution of gamma rays detected over the ship (lower, red curve) and at the dock (upper, blue curve).

Figure 17. Count rate in a single detector as a function of time for three different gamma energy regions as the detector is moved back from over the ship to the dock.......................................................... 31

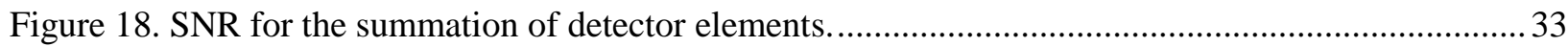

Figure 19. (a) Source, Background and total Counts for a source in position 7, depth=0\% ....................33

Figure 20. Optimum detectors in subset vs. cargo load with source at container bottom.......................... 36

Figure 21. Signal-to-noise ratio for the summation of detector elements............................................... 37

Figure 22. Optimum detectors in subset versus cargo load with source at different depths in a fully loaded

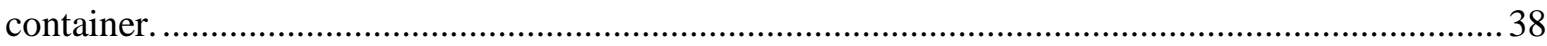




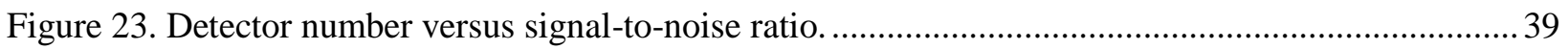

Figure 24. Plots of the results of several methods used for selecting optimal detector subset. ................. 40

Figure 25. Enumerated source locations used for the simulation. ....................................................... 41

Figure 26. Optimal detector subsets for SNR maximization, as chosen by annulus algorithm, several cargo

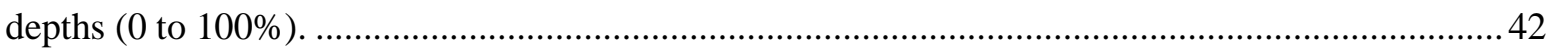

Figure 27. Results for best detector subset, with and without HEU test source (source at loaction 7 of Figure 25). Both plots are of the same data, with the right plot having a different y-axis range to show low level detail.

Figure 28. Overall Count Rate and Optimal Detector Subsets, included detectors for depth=0 shown in green. Top: Source Position 1, Middle: Source Position 7, Bottom: Source Position 14.

Figure 29. Average Algorithm data for Optimal Detector Subsets, for varying source depth, strength (Central source location shown). Top: Energy Distance Algorithm. Middle: Gross Count Algorithm. Bottom: KUT Algorithm.

Figure 30. Weight distribution of all containerized commodities entering the United States for 45 -foot containers (red curve) 40-foot containers (blue curve) and 20-foot containers (green curve)..........52

Figure 31. MDQ for various algorithms, with and without optimal subset method. ..............................54

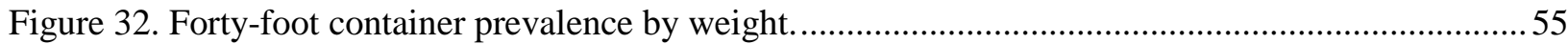

Figure 33. Forty-foot container prevalence by equivalent cargo thickness. ...........................................56

Figure 34. Simulated count rate as a function of source depth in the cargo for HEU...............................57

Figure 35. Maximum cargo thickness for HEU source detection. ......................................................57

Figure 36. Maximum Cargo thickness for HEU Source Detection, Worst Case ......................................58

Figure 37. Diagram of the model relating fractional cargo volume to maximum cargo depth for detection.

Figure 38. Plot of the fraction of a cargo container in which a source can be detected versus the maximum depth of detection of the source in the cargo container.

Figure 39. Plot of the signal rate from an HEU source in a load of wood as a function of depth of the source in the wood. The points are the MCNP model and the line is an interpolating function........68

Figure 40. Plot of the depth of an HEU source in wood as a function of the signal rate from the source. The points are the MCNP model and the line is an interpolating function.

Figure 41. Plot of the signal rate from an HEU source in an empty cargo container as a function of depth of the source in the container. The points are the MCNP model and the line is an interpolating function

Figure 42. Contour plot of the MDQ for an HEU source in a cargo container full of wood.....................71

Figure 43. Contour plot of the MDQ for an HEU source in an empty cargo container............................ 72

Figure 44. Plot of the fraction of TEUs as a function of average cargo density for cargo containers entering the U.S 
Figure 45. Plot of the fraction of TEUs as a function of cargo weight for cargo containers entering the U.S.

Figure 46. Plot of the fraction of TEUs as a function of the thickness of an equivalent wood cargo for cargo containers entering the U.S.

Figure 47. Plot of the cargo volume scanned as a function of the cargo weight for various HEU source masses.

Figure 48. Plot of the cargo volume scanned as a function of the HEU source mass.

Figure 49. Plot of the cargo volume scanned as a function of the HEU source mass. The blue curve depicts a linear dependence of signal on source mass while the red curve a $2 / 3$ power-law dependence.

Figure 50. Plot of the signal rate from an HEU source in a load of wood as a function of depth of the source in the wood. Points indicating the maximum depth for detection of a 10-kg source with the SBRD and with the Enhanced SBRD are shown.

Figure 51. Plot of the total detectable cargo volume scanned as a function of the MDQ of HEU for the case that the source is anywhere in the cargo.

Figure 52. Plot of the total detectable cargo volume scanned as a function of the MDQ of HEU for the case that the source is always on the bottom of the cargo container as a worst case. 


\section{List of Tables}

Table 1. Detector number of the four detectors closest to each source position....................................... 19

Table 2. Summary of Top 10 International Ports for the Three Major Container Sizes Shipped to the US .49

Table 3. Shipping Container Data Arranged by Major HS Code Categories ...........................................50 


\section{Introduction}

Radiation detectors are being deployed to scan containers coming into the US to interdict illicit trafficking of nuclear material [1]. Most of the containers from foreign countries are arriving by ship into the US, and detectors are being installed overseas at the ports of departure, as well as the US port of entry (POE). The typical container detector system is a radiation portal monitor (RPM), a two-sided system that the container passes through when arriving at, or leaving the port on a chassis pulled by a truck. Depending on the specific port workflow, some containers are scanned in the port itself, where yard haulers pull the container through the RPM. Other detectors systems are also being used to scan containers for radiation (rail portal monitors that scan loaded trains) or are in development (straddle carrier portal monitor systems).

An alternative scheme to scan for radiation is at the loading or unloading of the ship, or while the container is being moved in the port area by straddle carriers. These types of systems scan the container during the entire move and have longer times to detect the radiation than the typical pass-through RPM. Such systems typically consist of detectors mounted on a spreader-bar, which is the device that attaches to the top of the container and provides the interface between the port cranes or straddle carriers as shown in Figure 1. The radiation detectors studied here are mounted onto the spreader-bar, and come into close proximity ( $\sim 1$ foot or less) to the container. In order to monitor the entire container, multiple detectors are required to be mounted along the spreader-bar. The spreader-bars are designed to load various container lengths, from 20-foot to 53foot containers (although overseas containers are typically limited to $45 \mathrm{feet}$ ), by using hydraulic cylinders to 'spread' the bar out or pull it back to a shorter length. The radiation detectors must be mounted to accommodate this movement, and provide good coverage in various length positions. Since the spreader bar only comes into contact with the top of the container, the detection system is a single sided system, measuring radiation at the roof of the container.

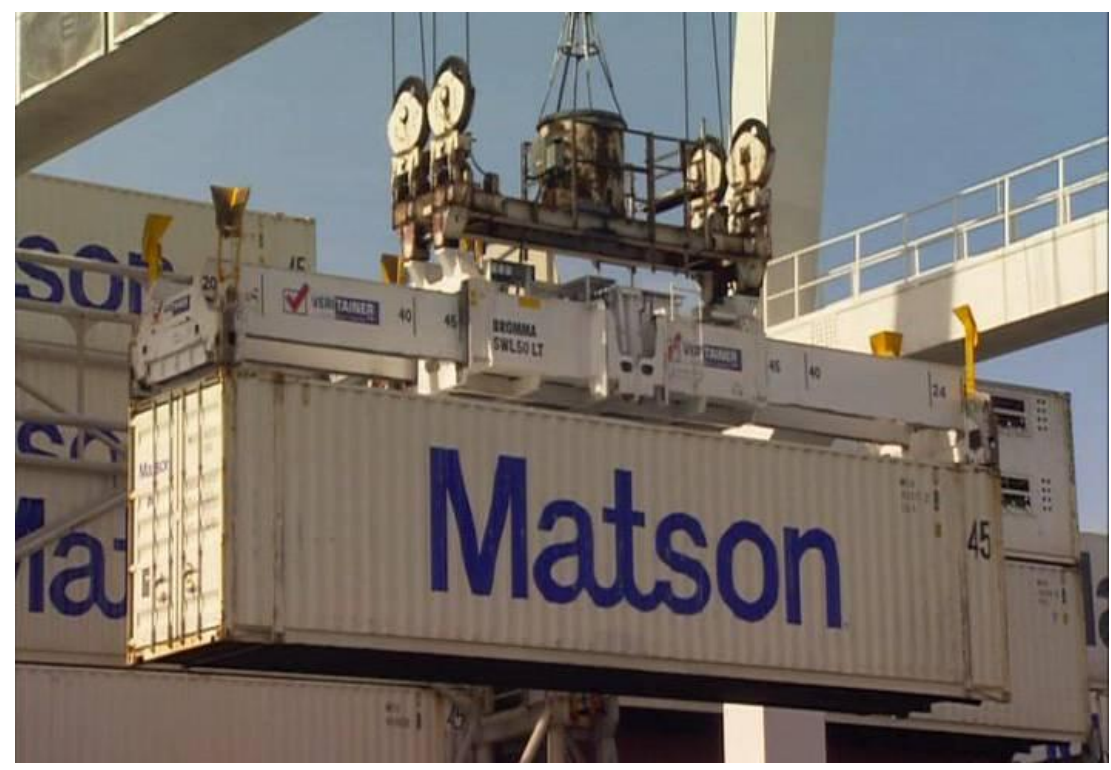

Figure 1. Picture of a spreader-bar (white bar) above the container, and attached to the crane block used for loading and unloading ships. Similar spreader-bars are used on straddle carriers.

There are several companies building such spreader-bar radiation detection (SBRD) systems. These companies have developed systems with gamma ray and neutron detectors, two types of radiation that are 
useful for national security applications. The companies typically use gamma ray detector material that can both detect gamma radiation and identify the radionuclides present. This can be very useful to discriminate threat material from naturally occurring radioactive material (NORM), and only trigger alarms and response from threat material. There are many NORM materials that are imported into the US, which are easily detected when in container-size volumes. Examples of typical NORM detected in containers at ports are ceramic tiles, granite sheets, and fertilizer.

\subsection{Prior Assessments}

A few commercial companies conducted field tests of initial designs for SBRDs almost a decade ago, primarily to investigate SBRD mounting and reliability issues [2]. Results of these tests suggested that detectors could be installed and operated in the challenging environment, and several companies developed full systems. Pacific Northwest National Laboratory (PNNL) also conducted a number of feasibility studies using modeling and simulation to access the viability of SBRD systems for radiation screening in US ports as part of the Radiation Portal Monitor Project (RPMP) [3-5].

Two commercial companies, VeriTainer and Bromma (in collaboration with Innovative American Technologies), provided SBRDs that were tested by U.S. Customs and Border Protection (CBP) in an operational environment at the Port of Tacoma in the summer of 2008 [6-7]. This test used a variety of containers with industrial sources inserted into a $7.5 \times 7.5 \times 8$ foot cubed wood block simulating cargo loads. The results of the testing indicated that both SBRDs possessed adequate neutron detection capabilities to meet CBPs requirements, but the gamma-ray detection capabilities of both were limited to a potential of detecting appropriate sources through only three to five feet of cargo, whereas a source could be located at the container bottom and necessitate detection through eight or nine feet of cargo. This depth limitation of the SBRDs is similar to a single side of an RPM, but the RPM has two sides that can provide the full eight feet of coverage. Both systems use thallium-doped sodium iodide $[\mathrm{NaI}(\mathrm{Tl})]$ as the gamma ray detector material, which is the appropriate material that can provide gamma ray identification and is both robust and available in large volumes. Additional detector material could likely be included in both systems to improve detection capability.

The U.S. Department of Energy (DOE) further tested these two systems in the fall of 2008 at Los Alamos National Laboratory (LANL) under the Second Line of Defense (SLD) program. Measurements were performed with special nuclear material (SNM) and NORM to quantify nuisance/false alarm rates (NFAR) as well as detection probabilities and investigate masking scenarios. The LANL and Tacoma test results were consistent in terms of the neutron and gamma-ray detection capabilities of these commercial SBRD systems. In addition, the LANL tests observed high nuisance/false alarm probabilities as well as a number of development challenges such as detector summing and gain stabilization that were not mature in the tested systems [8].

All of the assessments and testing indicates that the single-sided nature of the SBRD system limits the radiation detection capability, and cannot meet the same requirements as a two sides system such as a RPM, even if accounting for the long dwell and lower background. However, the SBRD system can provide radiation detection at a lower capability, which would provide some coverage for applications where RPM systems would not be feasible. For these possible scenarios, the SBRD system should be optimized to provide maximum capability. The testing also indicted that the commercial systems could be further optimized to provide a more capable system. This current work investigates the limits of radiation detection with SBRD systems, if properly optimized, which could provide a basis for the requirements that such a system would need to meet. 
The tests of the commercial systems in 2008 indicated possible improvements in a number of areas, including gamma ray detection and identification, and system robustness. Detection and identification capability could be increased by increasing the amount of detector material, as well as implementing more optimal alarm algorithms. Both systems use $\mathrm{NaI}(\mathrm{Tl})$ as the gamma ray detector material, which is the appropriate material that can provide gamma ray identification and is both robust and available in large volumes.

To help quantify the potential advantages of possible optimizations of the SBRD systems a collaborative effort between Sandia National Laboratory (SNL) and PNNL was initiated. PNNL managed the Tacoma test as well as conducted a number of assessments for the RPMP, and therefore had established expertise in gamma ray detection and identification. SNL was investigating detector robustness in the Extreme Environment Radiation Identification System project, which fit well with the robustness issue identified in the testing.

\subsection{Research Approach}

The initial study was a short-term investigation to determine if there was enough potential optimization to warrant further research and investigation. The first investigation was therefore constrained to primarily a modeling and simulation effort, where a model using additional detector material (compared to the commercial systems) was developed. This model was used to determine the radiation that deposited energy in the detectors for a variety of cargo loadings and threat source masses. Highly enriched uranium (HEU) was used as the threat source since it typically detected by the gamma ray signature only, and can be challenging to detect in cargo scenarios. The HEU was assumed to have ${ }^{232} \mathrm{U}$ present (taken to be $3 \times 10^{-8}$ ${ }^{232} \mathrm{U}$ by weight [9]), which makes it somewhat easier to detect due to an additional high energy gamma-ray, and is a reasonable approach.

The approach of the initial study was to model an average of the SBRD systems (as tested in 2008) and an enhanced model of an SBRD. This effort resulted in several presentations [10-11] that will be summarized here for the SBRD systems, and are described in more detail in Appendix B. The SBRD model used to represent the commercial systems was an average of the two commercial systems, and did not represent either system exactly. However, since the test results indicated similar performance, the average provided a representative model of the current (2008) commercially available capability. The enhanced SBRD, which will be referred to as 'enhanced SBRD (2010)', included additional detectors that could reasonably fit on a spreader-bar, leaving room for neutron detectors as well.

The HEU was modeled as 1-kg right circular cylinders, and up to 25 were simulated. The cylinders were assumed to be non-interfering (i.e., did not shield each other) with allowed for a simple linear scaling of signal with mass. The HEU source was located at the 20-foot plane (center) of a 40-foot container, which also included wood cargo. The wood was modeled with a density of $0.46 \mathrm{~g} / \mathrm{cm}^{3}$, which corresponds to the density of cargo that met the maximum cargo loading weight when filling the entire container volume. The wood was modeled as five layers, without any gaps or streaming paths.

For each thickness of wood cargo, a grid of 25 source locations (five vertical by five horizontal locations) was simulated at the 20-foot plane (center) of the 40-foot container. The detector response was simulated for each of the 125 simulations ( 25 source location in the 5 cargo thicknesses). To determine the minimal detectable quantity (MDQ) at each source location and cargo thickness, the results from the LANL test were incorporated. The energy deposited in the detectors is related to the minimal detectable quantity, but is convoluted by the data analysis, which is system dependent. Therefore, to provide the initial estimates, the 
LANL test results were used to convert detector responses into MDQ values. These results were used to provide the detector response required for minimal detectable quantities.

The conversion was also used with the enhanced SBRD (2010) simulation, with some additional capability given to the enhanced SBRD to account for some deficiencies in the commercial SBRD system's algorithms. The data from the Tacoma test was reviewed, and in many cases, the sources were visible in the energy spectra at cargo depths that did not trigger detection in the analysis software of the system. It was estimated that an improved algorithm would be able to detect sources that were visibly present in the spectra, with an estimated additional foot of cargo through which a source could be detected. Therefore, the enhanced SBRD (2010) incorporated more detector material and a lower threshold for the MDQ value.

Using the LANL test results, the simulated detector responses were converted into MDQs for each source location within the five cargo configurations. The MDQs were then grouped by the volume of cargo scanned for each HEU quantity (MDQ value) and cargo loading. For the volume calculated, the source was required to be within the cargo volume, that is, it couldn't be in the space above the cargo in a non-full configuration. The volume calculation allowed for an estimate of the percentage of cargo scanned for a particular quantity of HEU. These volumes and MDQs as a function of cargo loading were then convoluted with the actual distribution of US-bound containerized cargo. The containerized cargo distributions were obtained from a study performed by Lawrence Livermore National Laboratory (LLNL) [12]. These LLNL data are an average over 14 days at a limited number of ports, but were used to provide an estimate of the volume of the cargo container scanned as a function of the MDQ of HEU. This result can then be used to compare the capability of the SBRD and the enhanced SBRD (2010).

Figure 2 shows the results of the simulation and analysis for the estimated percentage of cargo scanned as a function of the MDQ. For example, if the target MDQ was $10 \mathrm{~kg}$ of HEU, the commercial systems (as of 2008 ) could detect this amount in about $30 \%$ of the incoming cargo volume. An enhanced SBRD (2010) is estimated to be able to detect the same $10 \mathrm{~kg}$ amount in about $65 \%$ of the cargo volume. The simulation indicated there were significant gains in capability that could be obtained by further optimization of the SBRD system. 
PNNL-21948

\section{HEU Source in Wood Cargo}

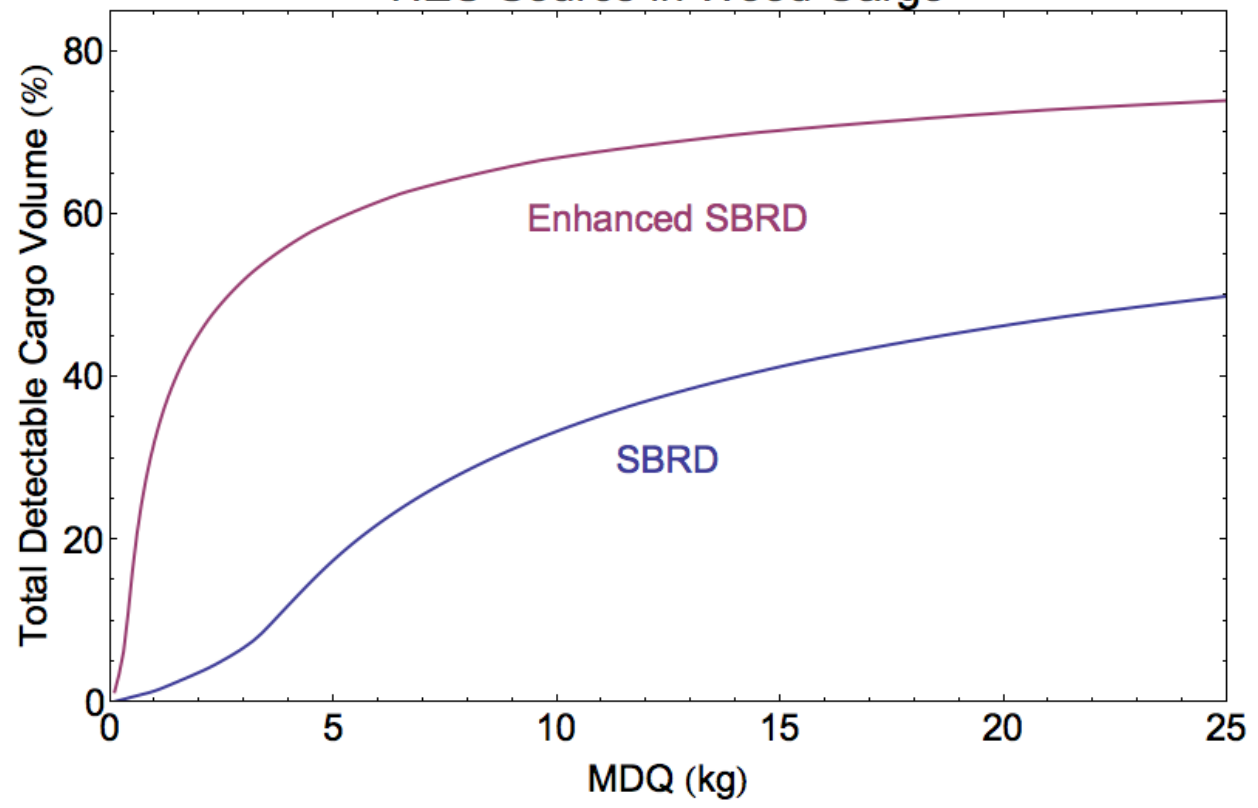

Figure 2. Total percentage of cargo scanned as a function of the targeted MDQ for the estimated commercial capability (2008) and enhanced SBRD (2010) for a random source location.

The limiting case was also evaluated, where the source was located on the bottom of the container for all cargo thicknesses as shown in Figure 3. As to be expected, the SBRD systems are less sensitive to the source in this location, since the detectors are above the container. As with the random source location results shown in Figure 2, the enhanced SBRD (2010) system has a significantly better performance.

With the promising results of this first investigation, a more involved research effort was undertaken. This effort was focused on resolving some of the ambiguities associated with the initial effort. The limitations were primarily associated with using the detection algorithms of the commercial systems (with an added estimated improvement factor). A more accurate representation of an enhanced SBRD capability could be obtained by building an actual system with enhanced capability and either direct measurements or, by validation of models, determine the detector response. Instead of relying on the commercial system detection capability as measured in the LANL testing, the analysis should use improved algorithms to provide actual detection limits. Therefore, as part of this investigation, research was performed on some promising algorithms for applications where a source is attenuated by significant amounts of cargo (deep shielding scenarios). A final limitation that was addressed was the distribution of cargo weight and density from the LLNL report. The report did not categorize the data by container length, and there is a significant difference between 20- and 40-foot containers in terms of weight distributions. The 20 -foot container has a loading capacity that is almost as large as a 40 -foot container. Therefore, a 20 -foot container can handle cargo with a higher density, and by being smaller, is a more efficient method to ship dense cargo that is weight limited. Having the distribution categorized into the container lengths would provide a more accurate representation of the cargo volume that is screened at a specific MDQ. 
PNNL-21948

HEU Source in Wood Cargo - Worst Case

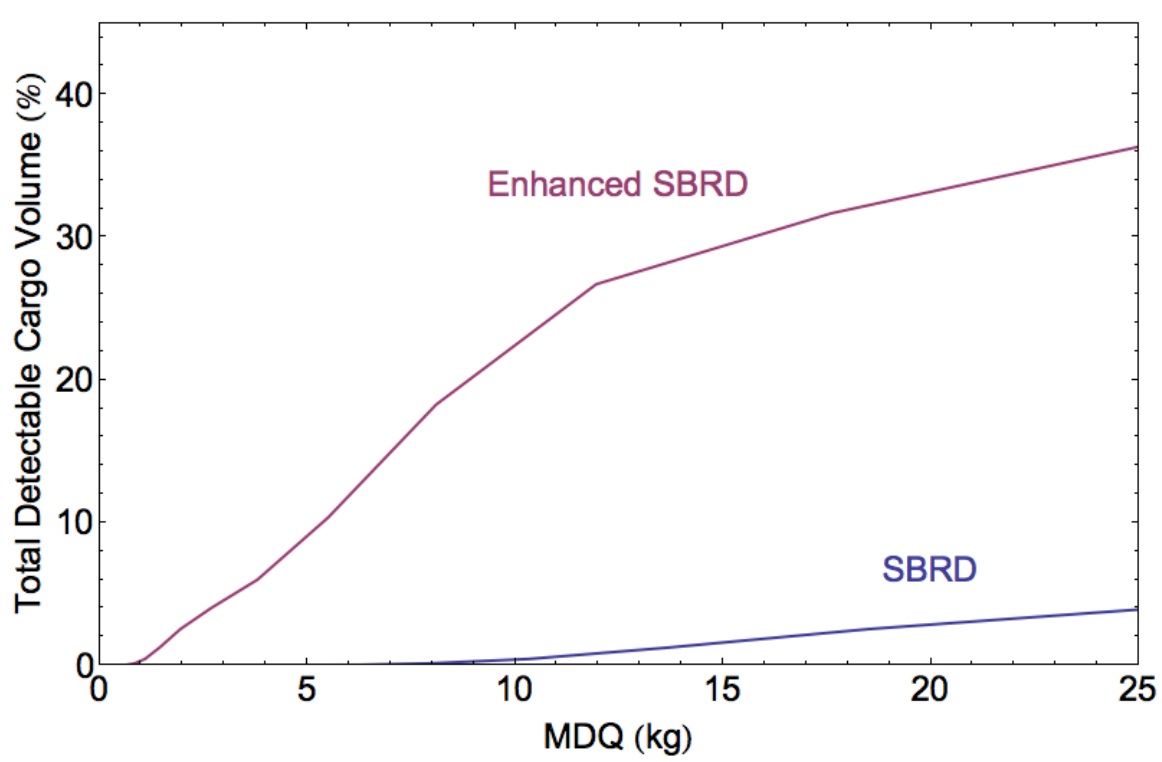

Figure 3. Total percentage of cargo scanned as a function of the targeted MDQ for the estimated commercial capability (2008) and enhanced SBRD (2010) for a source location on the container bottom.

The next phase of this research and investigation into the SBRD system and quantification of the possible improved capability with an enhanced SBRD is the focus of this report. The research was again a collaborative effort between PNNL and SNL, with PNNL taking the lead on the modeling and simulation and algorithm development, and SNL developing an enhanced SBRD system that could make actual measurements to validate the model and provide baseline capability. Originally the plan was to make measurements of most of the scenarios, and use simulations as needed, however, priorities have shifted and to date the measurements have served primarily to support model validation and to establish credibility for the simulations of the threat scenarios. Therefore, a significant simulation effort was undertaken to simulate HEU and additional sources including plutonium and depleted uranium, and to complete the analysis for both 20- and 40-foot containers. This report presents the results for HEU in a 40-foot container. Significant work was performed on both the algorithm development and the container cargo distributions entering the US, and that work will be presented along with the modeling, simulations, and validation effort. 


\section{Modeling and Simulation}

The modeling and simulation effort used the LANL developed Monte Carlo N-Particle (MCNP) radiation transport code to simulate the radiation transport and energy deposition in the detector material [13]. The physics engine in the simulation code has been well validated; however, it is very important that any specific model be validated against experimental measurements to ensure enough representation of the real system has been put into the model. For this effort, it was important to validate the model of the enhanced SBRD system against real measurements to verify the simulations were providing adequate consistency. A spreader-bar is a complex piece of machinery, and challenging to develop a model with enough details to simulate radiation transport representative of experimental measurements.

For this effort, the model was validated against measurements using the enhanced SBRD system developed and assembled by SNL. The model was then used to perform simulations of HEU in wood cargo to provide data that could be used as input into the algorithm development activity. The algorithms provided MDQ values for each modeled scenario for each algorithm, and the best results were convoluted with US-bound cargo distributions to provide an estimate of detection capability in terms of cargo scanned as a function of MDQ for HEU.

The SNL SBRD system uses 32 thallium-doped sodium iodide [NaI(Tl)] scintillators to detect the gamma radiation. Each $\mathrm{NaI}(\mathrm{Tl})$ detector is 2 inches in diameter by 18 inches long and mounted in a detector box with shock mounting to handle the rough handling encountered during the loading and unloading of containers.

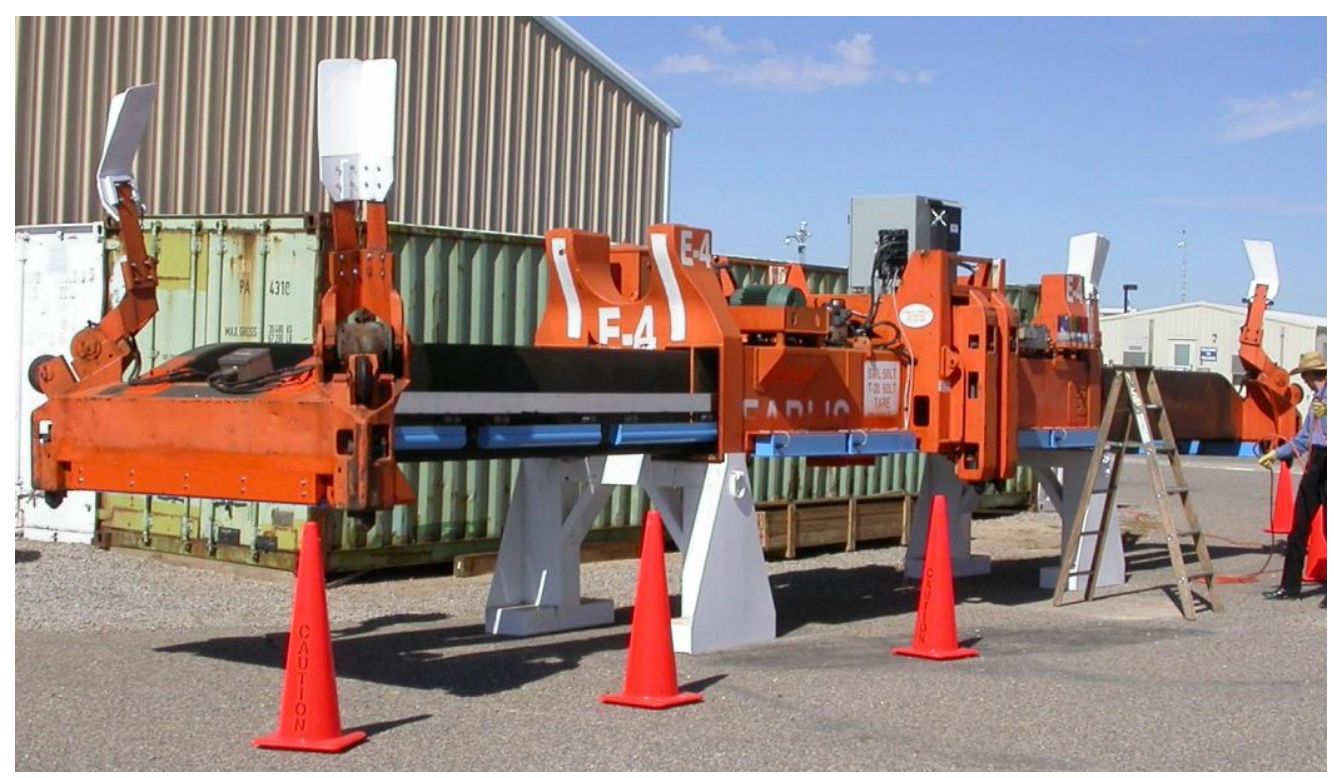

Figure 4. Photograph of the SBRD, before being placed on the 40-foot IMCC.

\subsection{Model Validation}

Experimental measurements were made at SNL on 3 November 2011 to validate the ability of the computer model of the SBRD to reproduce the functioning of the actual device. In these "zone- 
mapping" measurements, three radioactive sources were located together at various positions in the empty 40-foot inter model container (IMCC) on which the SBRD had been placed. The three sources were ${ }^{133} \mathrm{Ba},{ }^{137} \mathrm{Ba}$, and ${ }^{60} \mathrm{Co}$, and all placed together for each measurement. Figure 4 shows the SBRD before mounting on the IMCC. Some of the blue detector boxes, mounted to the side of the SBRD, are visible in the photograph.

Figure 5 shows the relative horizontal positions of the $32 \mathrm{NaI}(\mathrm{Tl})$ detectors in the SBRD and the 15 source positions in the empty 40 -foot IMCC during the zone-mapping measurements. The squares represent the detector positions and the circles are the source positions. The various detectors were mounted on the SBRD at three different heights depending on the mounting location: the main frame, the extension 'spreader' arms, or the end frame.

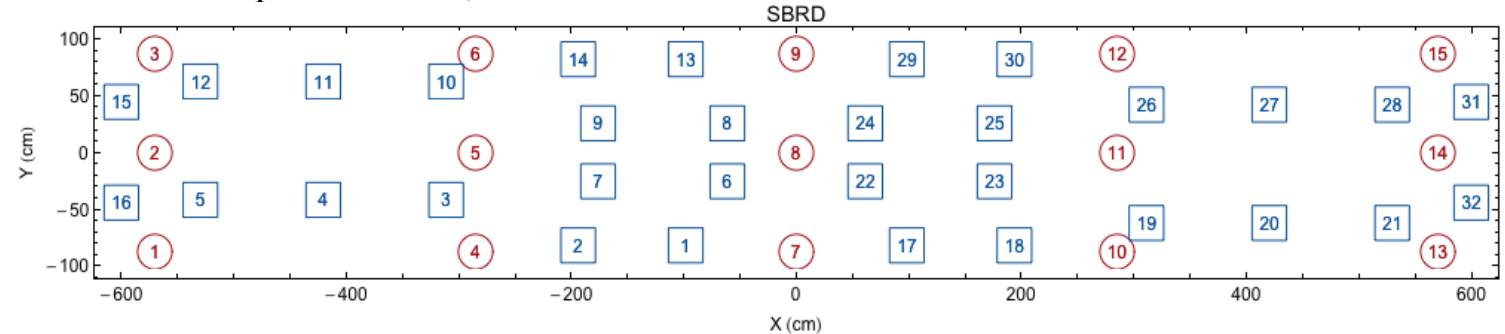

Figure 5. Relative horizontal detector positions (blue squares) and source positions (red circles).

For the measurements the sources were placed at two different heights for a total of 30 source positions. Source positions 1 through 15 had the source lying on the floor of the cargo container. Source positions 16 through 30 had the source held by a tripod at a height of about $130 \mathrm{~cm}$ above the floor of the cargo container, but at the same horizontal location as 1-15.

In order to keep the following comparisons of computer simulations to measured data manageable, comparisons are shown for the lower 15 source positions only and, at each source position, for the four detectors closest to that source position. Table 1 lists the four detectors in the SBRD closest to each source position. Those detectors are the same for the higher source position directly above each lower source position (i.e., source positions 16-30).

Table 1. Detector number of the four detectors closest to each source position.

\begin{tabular}{|ll|llll|}
\hline $\begin{array}{l}\text { Source } \\
\text { Position }\end{array}$ & \multicolumn{4}{|c|}{$\begin{array}{l}\text { Nearest Detectors } \\
\text { Numbers }\end{array}$} \\
\hline 1 & 16 & 16 & 5 & 15 & 12 \\
2 & 17 & 15 & 16 & 5 & 12 \\
3 & 18 & 12 & 15 & 16 & 5 \\
4 & 19 & 3 & 2 & 7 & 4 \\
5 & 20 & 3 & 10 & 7 & 9 \\
6 & 21 & 10 & 14 & 9 & 3 \\
7 & 22 & 6 & 22 & 1 & 17 \\
8 & 23 & 6 & 8 & 22 & 24 \\
9 & 24 & 8 & 24 & 13 & 29 \\
10 & 25 & 19 & 18 & 23 & 26 \\
11 & 26 & 26 & 19 & 23 & 25 \\
12 & 27 & 26 & 30 & 25 & 27 \\
13 & 28 & 21 & 32 & 31 & 28 \\
14 & 29 & 31 & 32 & 28 & 21 \\
15 & 30 & 31 & 28 & 32 & 21 \\
\hline
\end{tabular}


The following plots compare the simulations of the zone-mapping measurements for the four closest detectors to each of the lower set of source positions to the measured data. In each plot, the measured spectrum is shown in red and the simulated spectrum in blue. The measured spectra were energy calibrated using the lines of the ${ }^{133} \mathrm{Ba},{ }^{137} \mathrm{Ba}$, and ${ }^{60} \mathrm{Co}$ sources evident in the spectra as well as the lines from ${ }^{40} \mathrm{~K}$ and ${ }^{208} \mathrm{Tl}$ in the background. Based on the energy calibration, each measured spectrum was re-binned to $5-\mathrm{keV}$ bins. The simulated spectra were also calculated in 5-keV bins. The plots are divided into five groups of three source positions, moving from the left end of Figure 5 to the right end. The first group of plots shown in Figure 6 gives the results for source positions 1 through 3 .
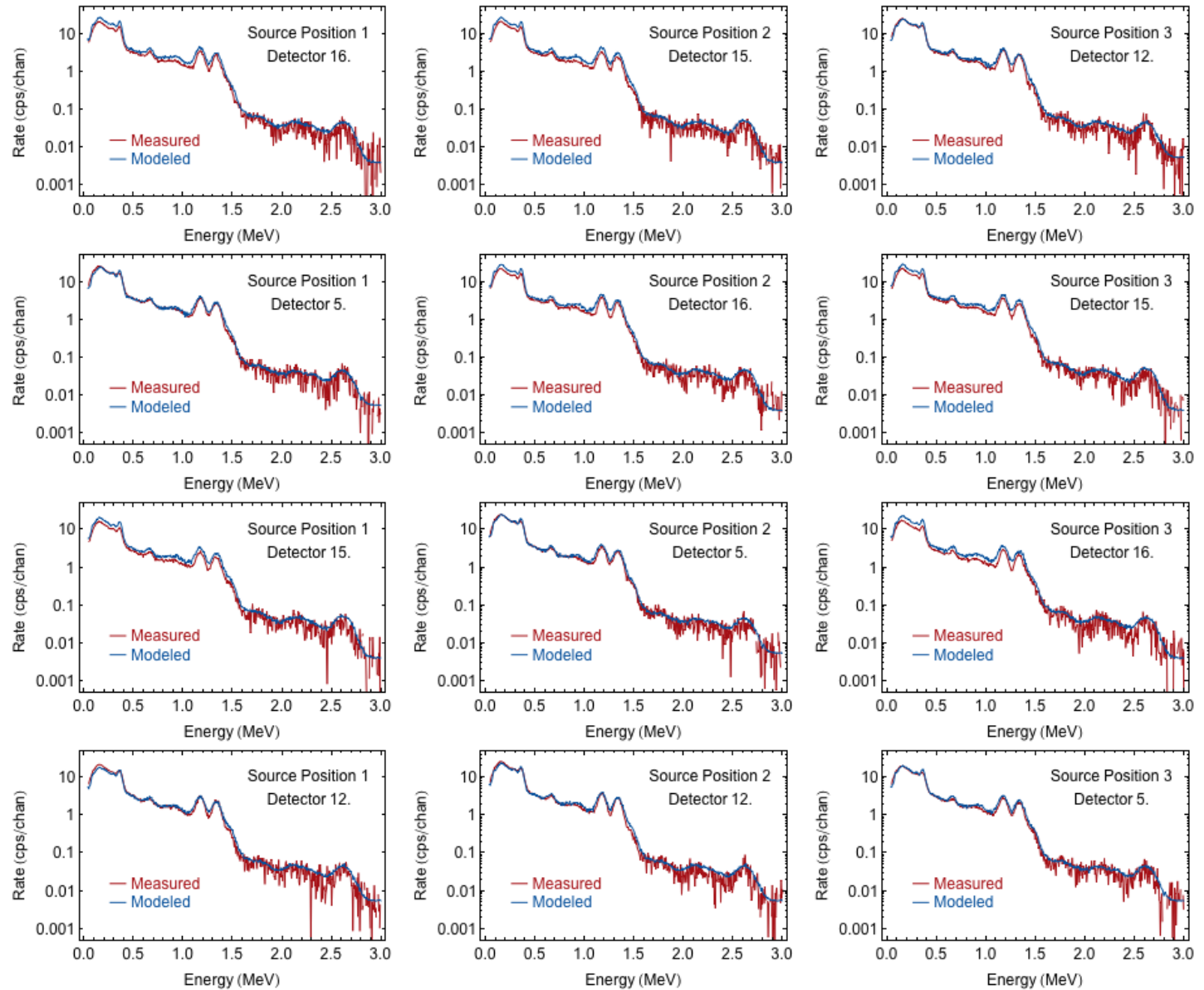

Figure 6. Comparison of simulated to measured spectra for source positions 1 through 3.

Figure 7 gives the results for source positions 4 through 6 and Figure 8 gives the results for source positions 7 through 9. Source positions 10 through 12 are shown in Figure 9 and finally, Figure 10 gives the results for source positions 13 through 15. The agreement is quite good for most of the sources, but it is challenging to quantify. Therefore a difference between the simulated and measured data was computed and normalized to have a fractional difference as a function of energy to be able to more quantitatively compare the simulations with the experimental measurements. 

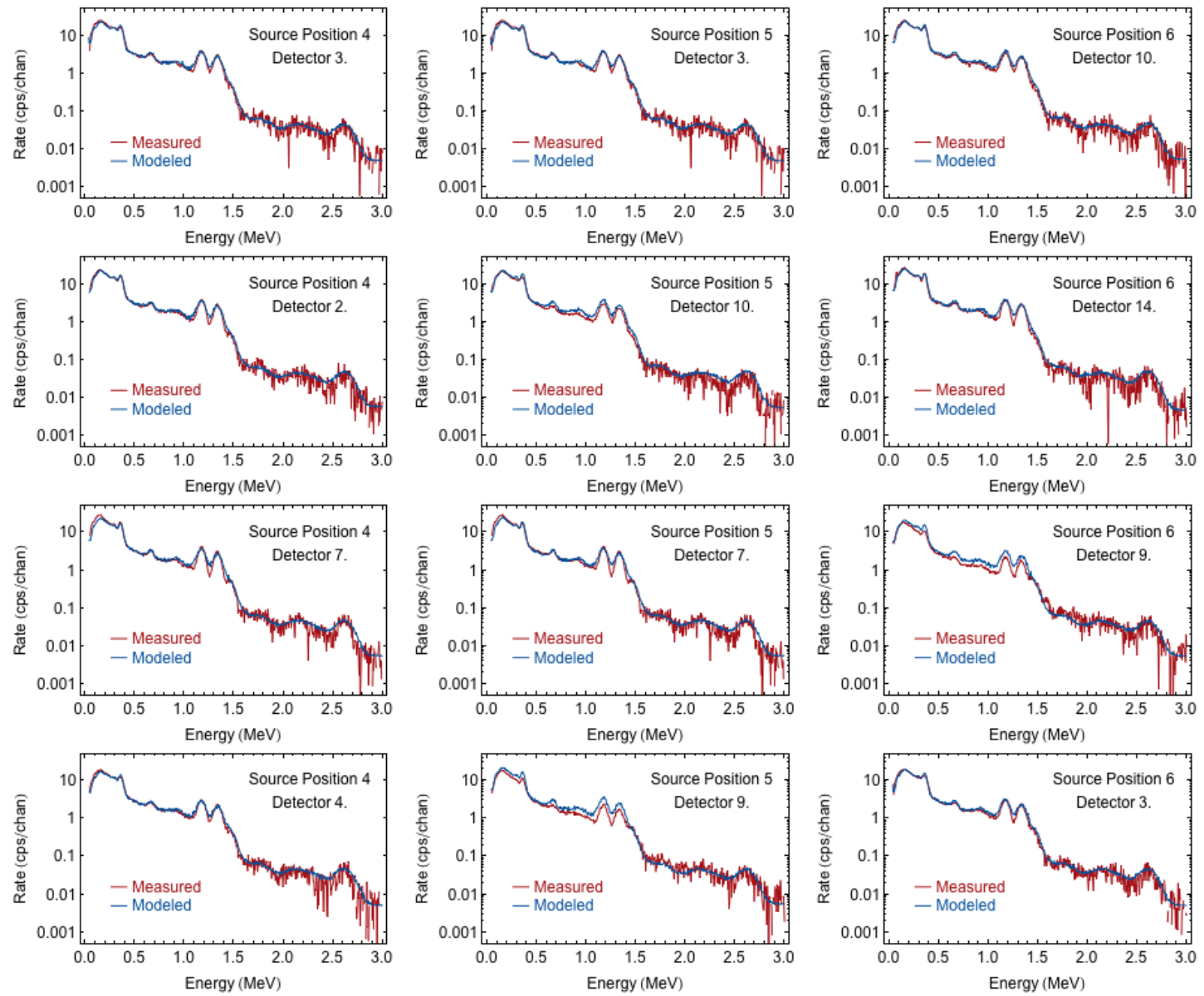

Figure 7. Comparison of simulated to measured spectra for source positions 4 through 6 . 

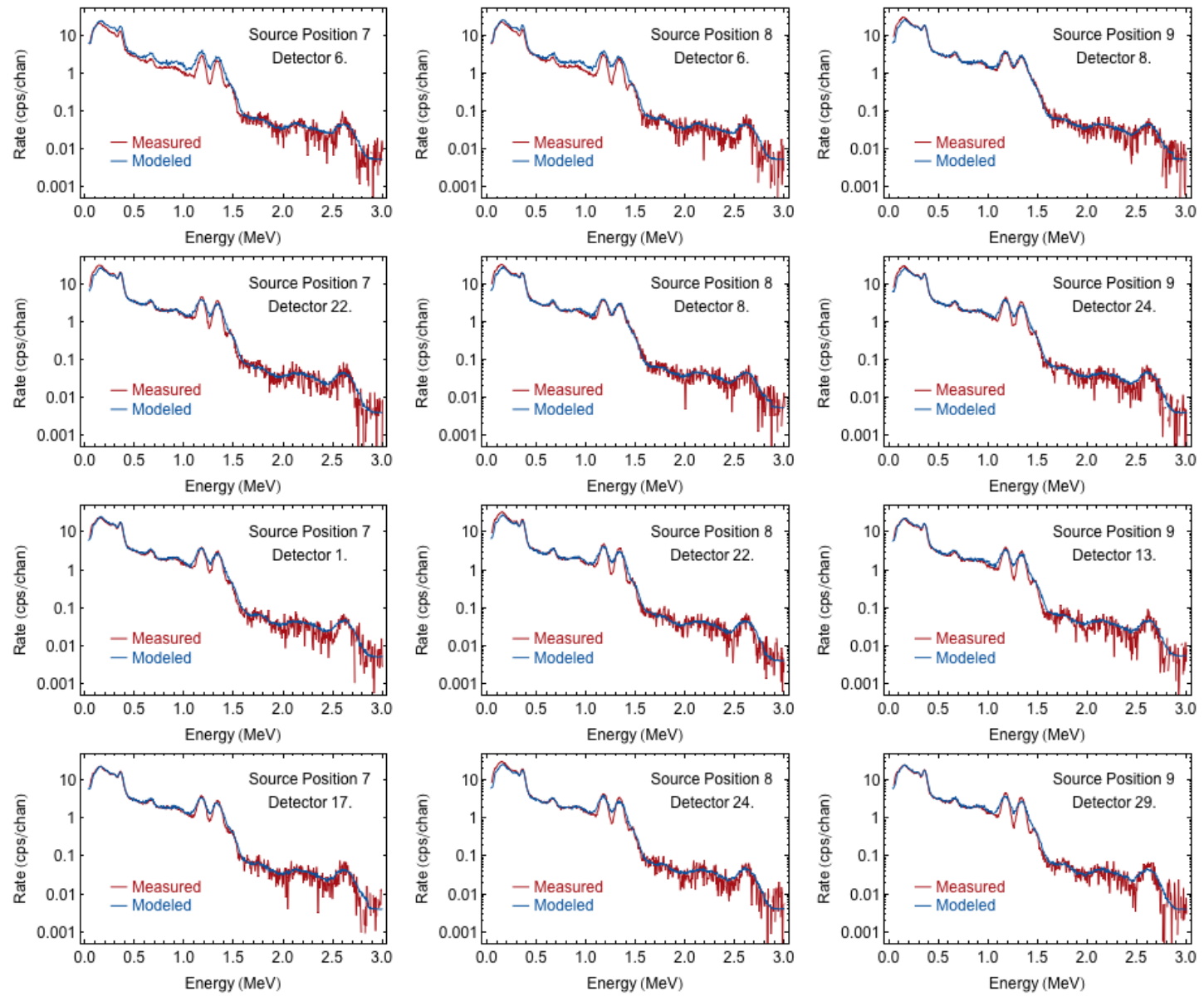

Figure 8. Comparison of simulated to measured spectra for source positions 7 through 9. 

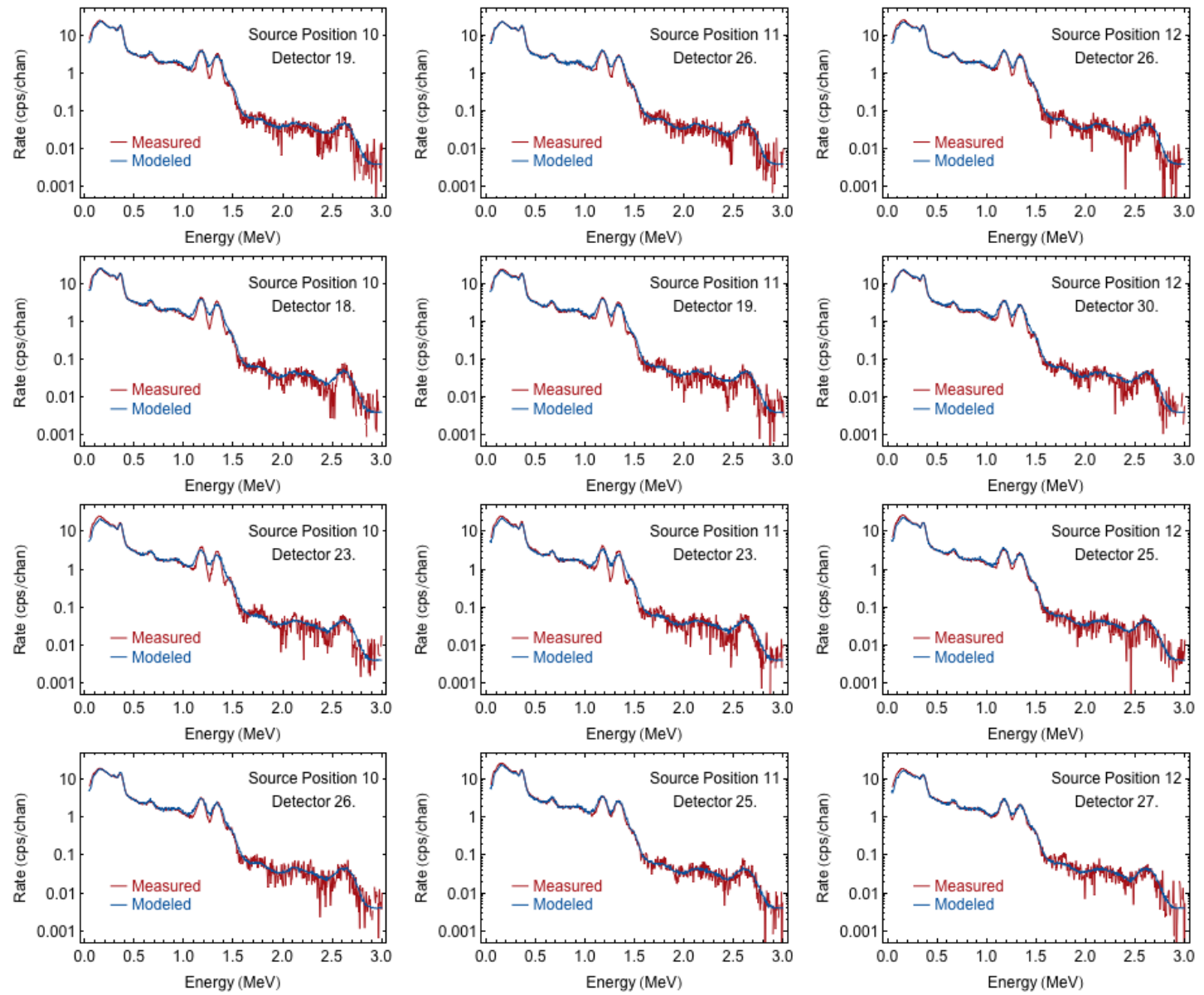

Figure 9. Comparison of simulated to measured spectra for source positions 10 through 12. 

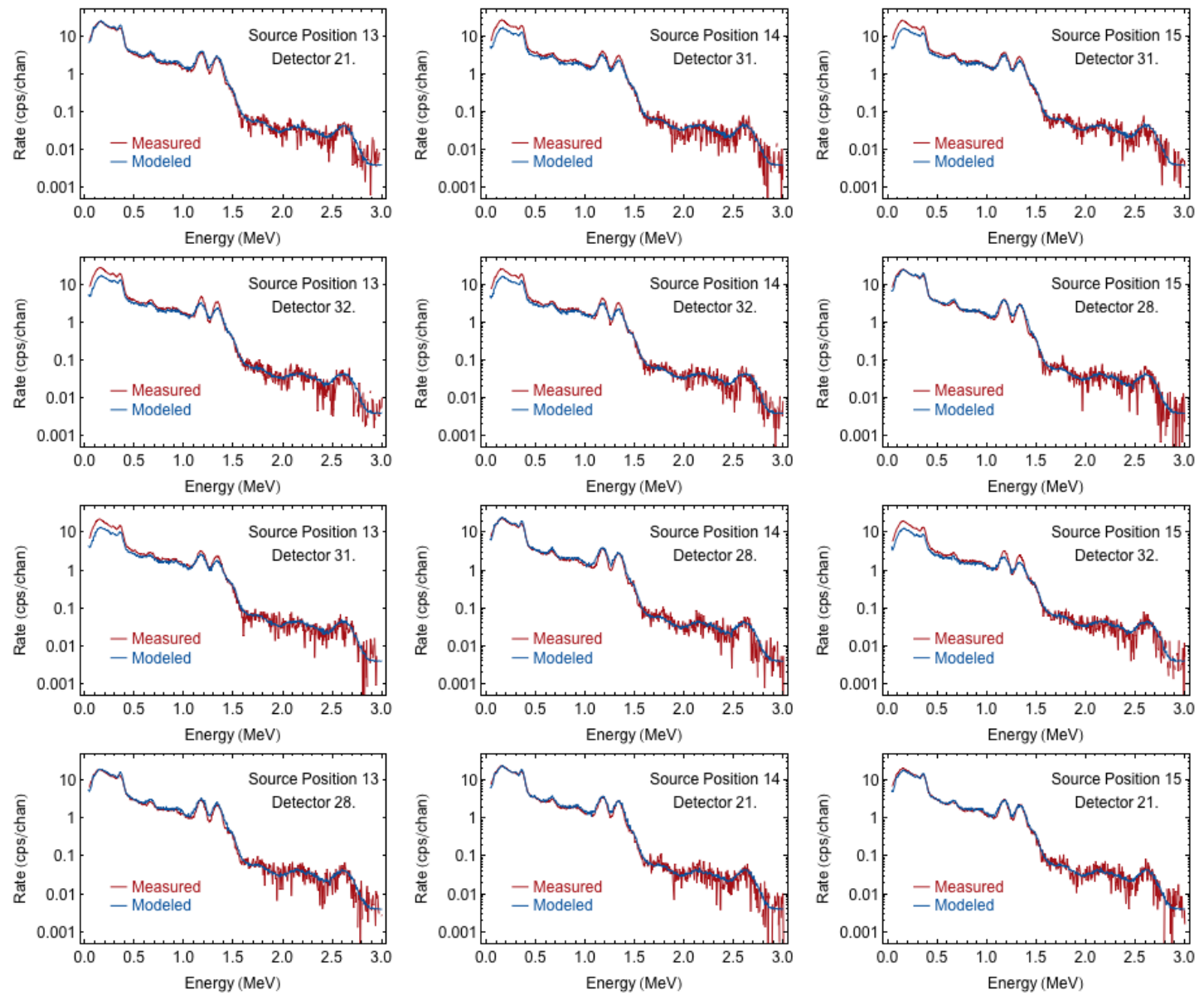

Figure 10. Comparison of simulated to measured spectra for source positions 13 through 15.

Figure 11 show the fractional difference $\left(S_{i}-M_{i}\right) / M_{i}$, with $S_{i}$ the counts in channel $i$ of the simulated spectrum and $M_{i}$ the counts in channel $i$ of the measured spectrum, between the simulated and measured spectra for the four detectors nearest to each source position. The differences are plotted up through the energy of the highest strong transition, the ${ }^{208} \mathrm{Tl}$ transition at $2.6 \mathrm{MeV}$. Above that energy both the measured and simulated data have few counts and are dominated by statistical fluctuations. 

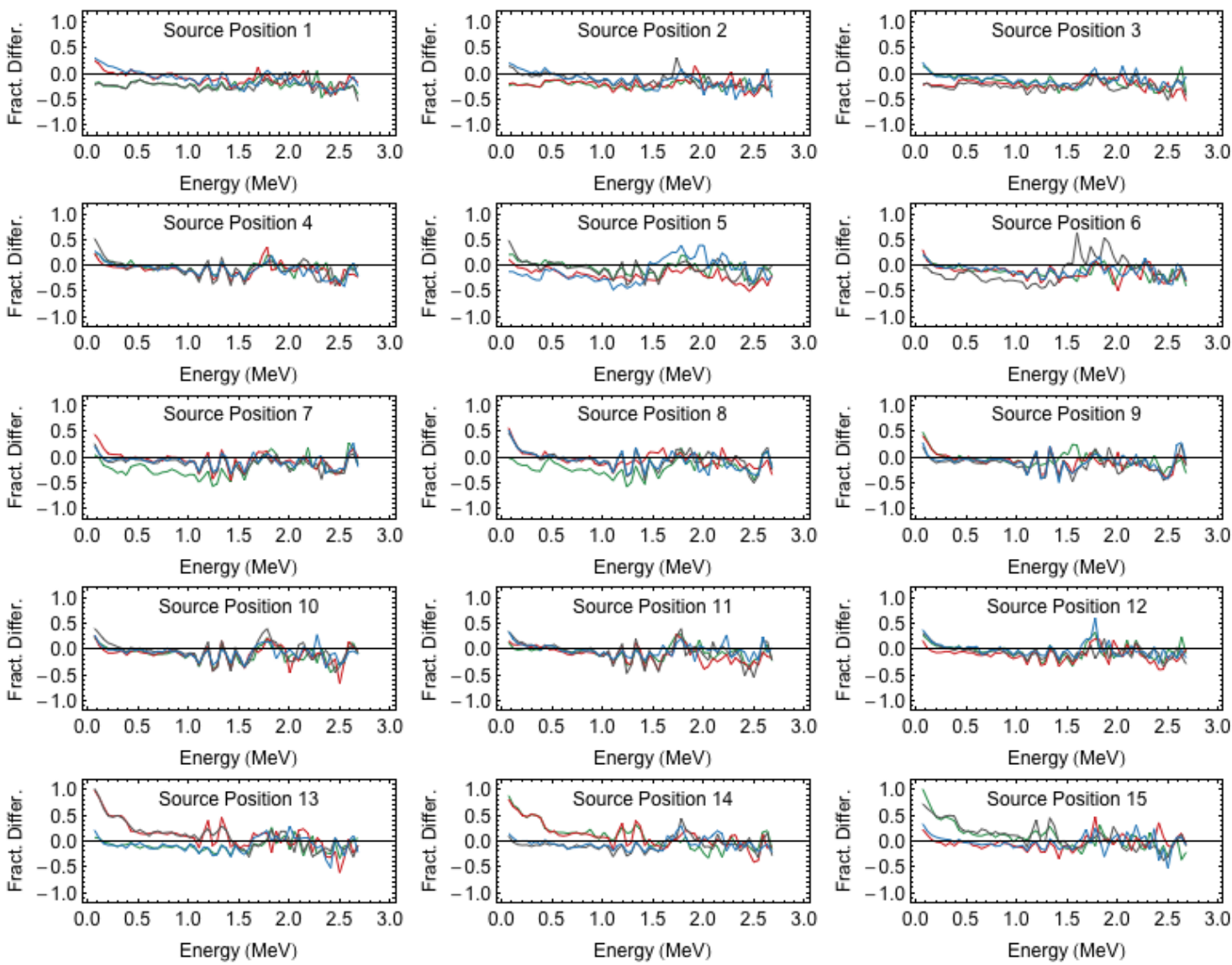

Figure 11. Plots of the fractional difference between the simulated and measured spectra for the four detectors nearest to each source position.

All the curves were combined together and are shown in the Figure 12, where it can be seen that they form four groups. Most of the curves, shown in blue, have a very similar pattern that clusters around zero. These curves correspond to measurements involving 26 of the 32 detectors. The six curves, shown in red, where the simulations are low compared to the measurements (at the left end of the plot) are all the measurements involving the detectors 15 and 16, which are located all the way at one end of the SBRD (see Figure 5). Similarly the six curves, shown in green, where the simulated results are high compared to the measurements (at the left end of) the plot are all the measurements involving the detectors 31 and 32, which are located at the other end of the SBRD. The four curves, shown in gray, that are also low on the left involve the two detectors 6 and 9, which are located in the middle of the SBRD. It is not known why these six detectors out of 32 are less well reproduced by the model. 
PNNL-21948

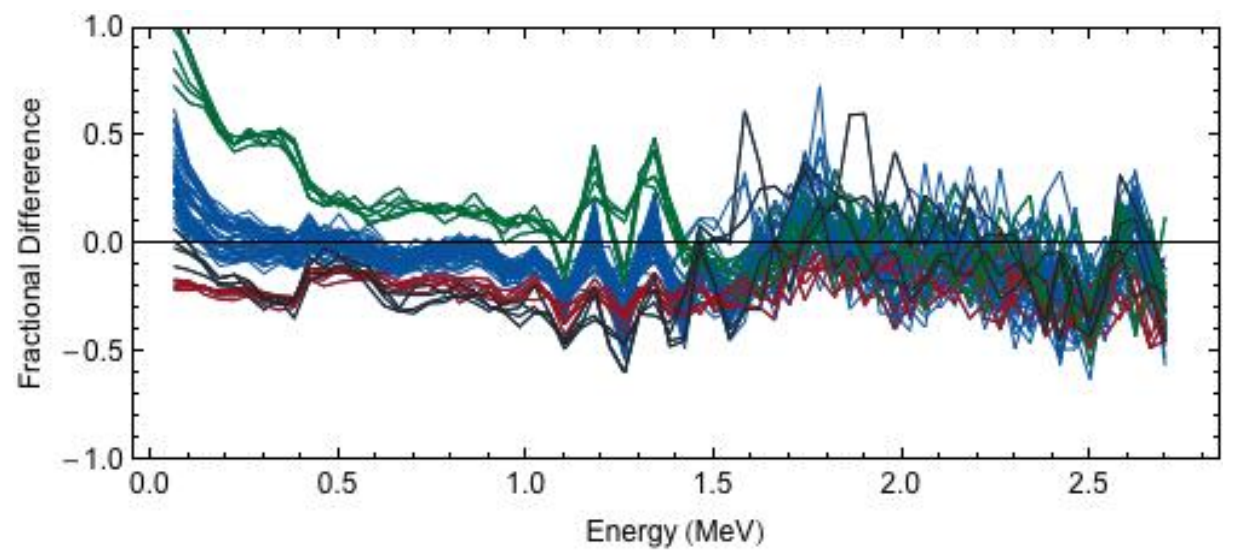

Figure 12. Plot of the fractional difference between the simulated and measured spectra for the four detectors nearest to each source position for source positions 1 through 15.

The standard deviation of the fractional differences between simulated and measured spectra for the four detectors closest to each of source positions 1 through 15 range from about $10 \%$ to $25 \%$. For the subset of 26 detectors shown in blue in Figure 12, the standard deviation range from about $10 \%$ to $20 \%$. This is reasonable agreement for a computer model of this complexity, and is consistent enough to provide good data from estimation of the SBRD capability. Although better reproducibility may be achievable by adding additional features into the model, it would require significant additional effort.

\subsection{Threat Scenario Simulation}

Once the validation was completed to a sufficient level, simulation of threat material contained within cargo in the IMCC was made. Therefore only a single material, HEU, was simulated in a single 40foot container, in keeping with the change in priorities of this project. As with the previous investigation, wood with a density of $0.46 \mathrm{~g} / \mathrm{cm}^{3}$ was used to simulate cargo in the container, to provide for a complete validated model to describe data from the HEU source and background with intervening cargo. This is the density that would constitute the maximum weight that a normal 40foot container could carry (58000 pounds), if the cargo container was completely filled. It is possible to ship more dense material, but the cargo could not then fill the container before reaching the maximum weight. Gamma ray attenuation effects due to the atomic number of the material are relatively small if the aerial density of the cargo does not change. That is, the same weight of cargo, spread evenly across the floor would attenuate gamma rays to about the same extent, whether it was a low-density material filled to the top of the container, or a high-density material only partially filling the container. This allows a single cargo (the wood at a density of $0.46 \mathrm{~g} / \mathrm{cm}^{3}$ ) to provide a good simulation of all cargo even with different densities, by using equivalent weight cargo loading. With this approach, only the cargo weight needs to be known, and greatly reduces the complexity of the overall estimation, although some fidelity (at the $\sim 10 \%$ level) is lost due to the effects of the atomic number of the cargo. However, the loss of fidelity greatly simplifies the complexity of the calculations and allows a reasonable estimation. By using a single cargo and focusing on the cargo weight, the results can easily be combined with the cargo weight distribution of containers carrying US imports in order to report the results as fractions of the amount of cargo entering the US. 
Figure 13 shows a simulation for the energy spectra recorded in a $\mathrm{NaI}(\mathrm{Tl})$ detector located just above the middle of the top of the cargo container for the background radiation coming from the asphalt below the cargo container. The container is filled with various thicknesses of wood, indicated as percentage of a full container.

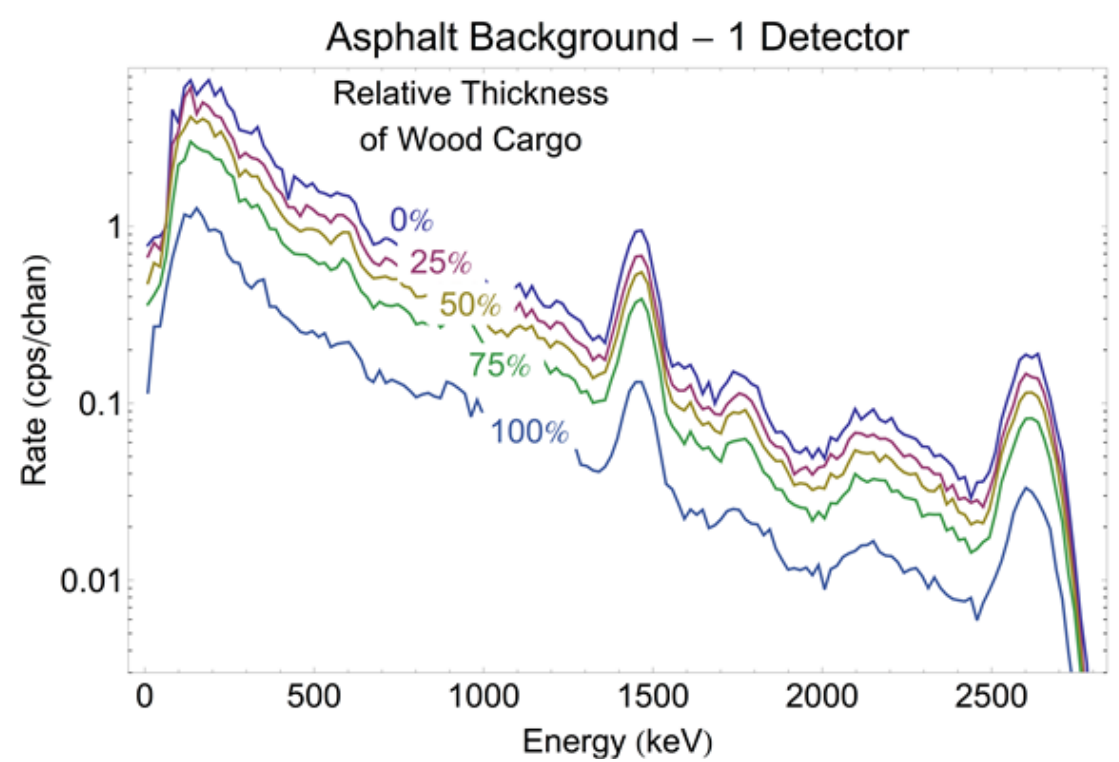

Figure 13. Simulated spectra in the central detector for the asphalt background. 


\section{Algorithm Development and Characterization}

One of the shortcomings of the initial investigation was the reliance on the commercial SBRD algorithms. It was observed in both the CBP tests at Tacoma, and the SLD testing at LANL, that the commercial vendor's algorithms could be improved. For the initial investigation, an improvement factor was estimated based on spectra from the CBP test, but was not validated, and therefore was an area of investigation for the follow-on study.

For this investigation, a significant effort was expended on the development and testing of improved data analysis approaches for SBRD systems. Several aspects of data analysis pertinent to optimal detection were investigated, including the optimal scanning time, the optimal subset of detectors to include in the analysis, and the optimal detection algorithm for source detection in deeply shielded scenarios. The detection algorithms investigated for this analysis had been developed previously, but were optimized or re-implemented for this application. These algorithms consisted of a number of detection and identification algorithms including gross counting [14], energy distance (anomaly detection using a principle components approach) [15], peak finding [16], Gamma Detector Response and Analysis Software (GADRAS) [17]. In addition, several algorithmic approaches are in development at PNNL and are used here, including limited GADRAS (anomaly algorithm using a library with only naturally occurring isotopes), KUT (anomaly algorithm using potassium, uranium, and thorium components) and QuID (an isotope identification algorithm utilizing a novel template matching approach) [18].

\subsubsection{Optimal Scan Time}

The SBRD system allows for a long dwell time for scanning of the container, since the detector can measure the container for the length of time the spreader-bar is attached to the container for movement. For loading or unloading a ship, this time is about 60 seconds on average, allowing for approximately 30 containers to be loaded or unloaded each hour (total cycle time is $\sim$ two minutes). There can be shorter times for containers that are loaded onto, or offloaded from ship locations nearer the dock, whereas containers deep in the hold with take longer than the average. And the time could be much longer, for example, when a straddle carrier moves the container in the lay-down yard.

The background observed by the SBRD system can change significantly during the move, particularly in loading and unloading ships. The background arises from naturally occurring radioactive isotopes primarily in the soil, specifically, from potassium-40, and the uranium and thorium decay chains. Some background is also expected from NORM contained in cargo onboard the ship itself. While this background is not explicitly included in the simulations due to its unpredictable nature, it is expected that it will impose a minimum distance of spreader-bar approach beyond which no data will be taken, in practice. Additionally, some component of the background comes from cosmic rays. The naturally occurring isotopes are present but to a lesser degree in water, and so the background is significantly less when a detector is over the water, by as much as a factor of ten. Typically, the spreader-bar and container do not move over open water, as the ship is tied to the dock, and therefore the background changes are less than when moving over open water. However, the backgrounds can still change by factors of two to three moving between ship and dock. One of the ideas for increasing capability is to maximize the signal-to-noise ratio (SNR), which is basically the detector net signal 
(minus background) divided by the square root of the background, by selecting only the data that is associated with low background.

Measurements were made with a single $\mathrm{NaI}(\mathrm{Tl})$ detector on a spreader bar at the Port of Tacoma in June 2010. The detected gamma ray signal for a large number of movements of the spreader bar carrying a container from a ship back to the shore is shown in Figure 14. The signal starts out low above the ship and increases by nearly a factor of three as the detector moves over the asphalt of the dock. There is a great deal of variation container to container, but the average signal, shown as a dashed line, follows a simple sigmoid form. Also shown by dotted lines are the one-standarddeviation limits for the distribution of signal. Under the assumption that most of these containers did not hold radioactive sources, this represents the background that would be seem in a gamma ray detector mounted in a spreader bar as it moves from the ship to the shore.

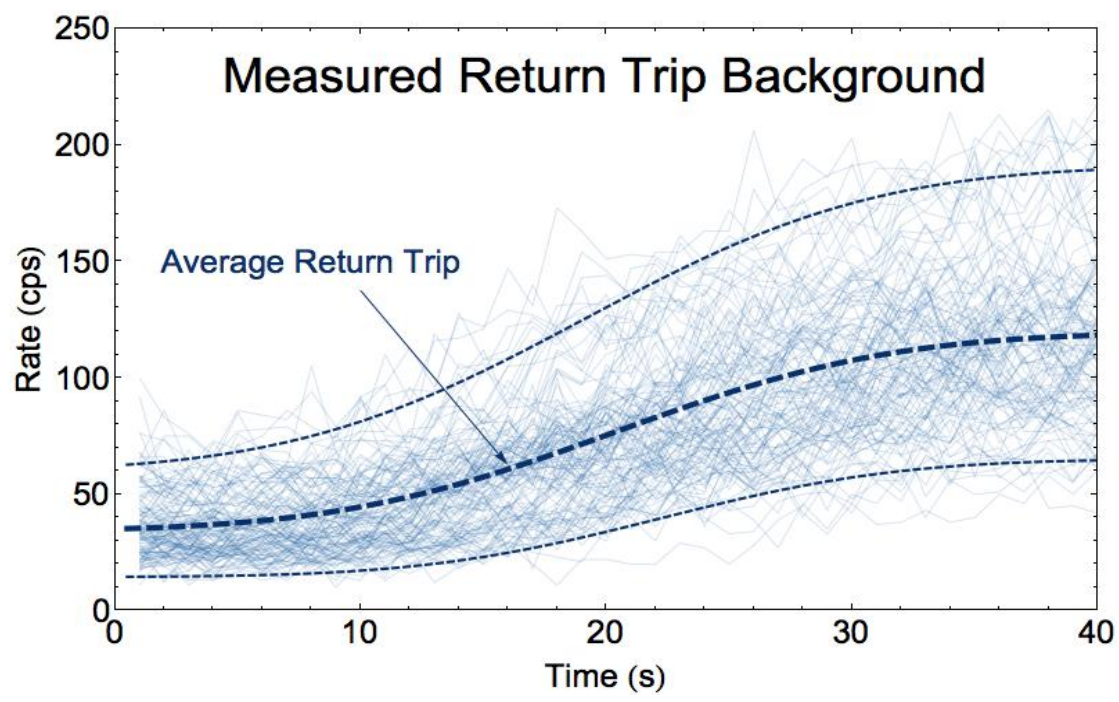

Figure 14. Count rate in a single detector as a function of time as the detector is moved back from over the ship to the dock.

Using the average background dependence, one can calculate the signal-to-noise ratio for a given source signal and different detection schemes. The first question to be asked is what the optimum measurement time is. The two extremes are summing the measurement over individual time intervals or over the entire time. This comparison is shown in Figure 15 for the signal-to-noise ratio calculated for the sum over all detected gamma energies. The blue curve shows the case of individual, onesecond measurements. As can be seen, the signal-to-noise ratio for each one-second measurement decreases as the spreader bar moves back over the dock and the background increases.

On the other hand, the red curve shows the total, cumulative signal-to-noise ratio for a measurement that sums the signal (source plus background) for the time the spreader bar leaves the ship to a later time $t$ as a function of that time. As can be seen, the total signal-to-noise ratio continues to rise for all times out to that when the spreader bar is back over the shore. This indicates that the optimal measurement time as determined by signal-to-noise ratio is the entire time from ship to shore even though the signal-to-noise ratio for small time intervals is decreasing as the spreader bar moves toward the shore. 
PNNL-21948

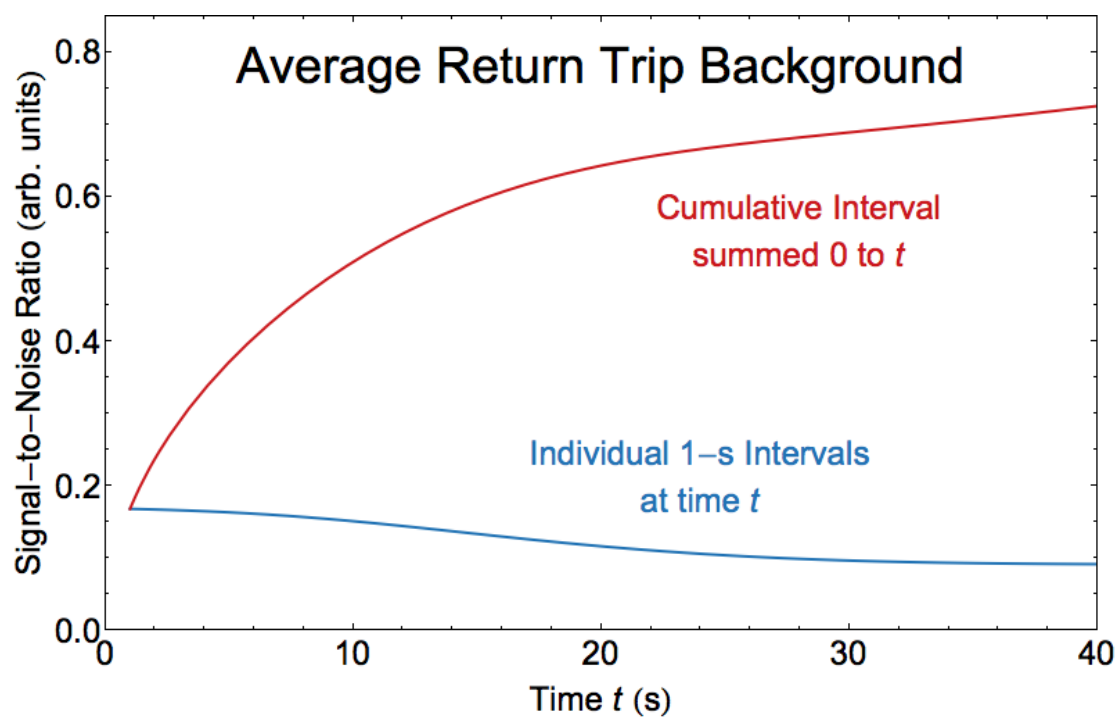

Figure 15. Signal-to-noise ratio in a single detector as it is moved back from over the ship to the dock. The lower, blue curve is calculated for separate, one-second intervals while the upper, red curve is calculated for the entire cumulative time.

At the same time that the overall count rate is changing as the detector mounted in the spreader bar moves from the ship back over the shore, the energy distribution of the detected signal is also changing. This is shown in Figure 16, which shows the spectra taken when the detector and container are over the ship, in red and over the shore, in blue.

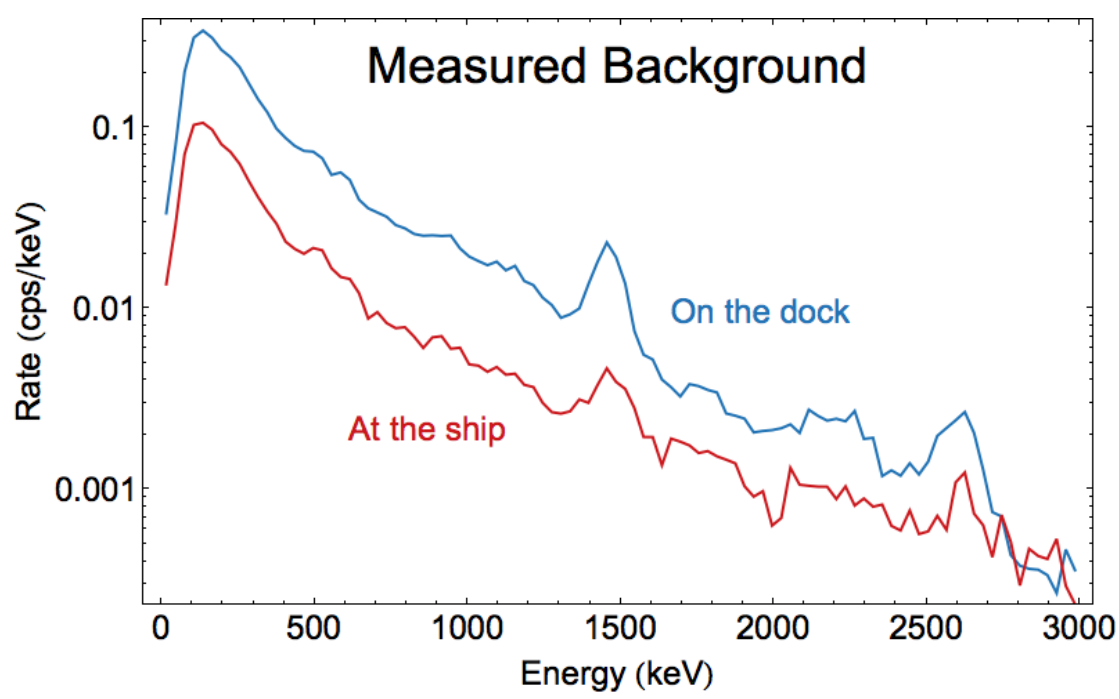

Figure 16. Energy distribution of gamma rays detected over the ship (lower, red curve) and at the dock (upper, blue curve).

This is also shown in Figure 17, which shows measured background for the return trip from the ship to the dock for three different gamma ray energy regions. The three curves are normalized to a value of one at the initial time above the ship and give the rate relative to that value as the detector is moved 
back over the dock. The upper, red curve is for gamma ray energies up to about $1.6 \mathrm{MeV}$, or just above the potassium-40 emission line (at $1460 \mathrm{keV}$ ) visible in the spectra of Figure 16. It increases by about a factor of three during the movement to the dock. The middle, blue curve is for gamma ray energies from there up to about $2.9 \mathrm{MeV}$, or just above the thallium-208 emission line (at $2614 \mathrm{keV}$ ), also visible in Figure 16. It increases by only a factor of about two during the return trip. The lower, gray curve is for higher gamma ray energies (mostly cosmic rays). It stays essentially constant. This indicates that alarm algorithms that are sensitive to the energy distribution of the gamma rays may have a somewhat more complicated dependence on measurement time. This should be investigated in more detail.

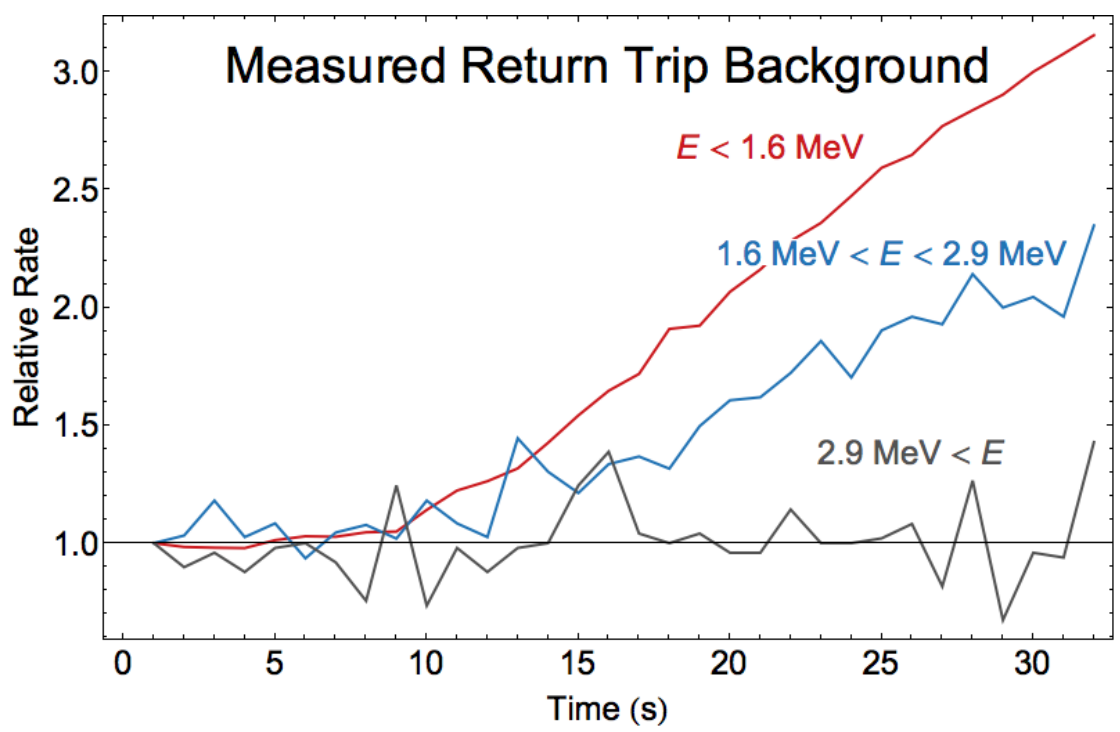

Figure 17. Count rate in a single detector as a function of time for three different gamma energy regions as the detector is moved back from over the ship to the dock.

\subsubsection{Optimal Detector Subset}

To simulate optimal use of the detector array, the optimal number of detectors to be used (i.e., summed together in a single measurement) has been investigated. In general, using only one detector element (and applying the algorithm in question only to the single crystal with the highest count rate) will lose resolving power of the instrument, while always summing every detector together will include too much background and reduce sensitivity. Therefore, some "optimal number" between these extremes is sought.

In the previous data generation stages, we have assumed a set of sensors arrayed above a container filled with a uniform material and containing a point source at a given depth. We furthermore assume that the sensors are identical and independent. To improve detection, we add the measurements across the sensors before applying a detection algorithm. Sensors nearest the source will provide the highest signal-to-noise ratio (SNR). Adding their measurements will increase total SNR. Assume for this discussion that the measured background is the same for every detector. As distance from the source increases, sensors see less and less source but the same background, and so eventually adding measurements from sensors far from the source will decrease SNR. If we simply add measurements, a 
key question is where the cutoff is in terms of sensors to include in the measurement sum, by identifying the set of sensors whose sum provides maximal total SNR.

For this study, it is assumed that the detector most closely approximating the source's location in the cargo is known from inspection of the relative count rates in each crystal. Then, the optimal number of detectors to sum is defined to be that which yields the greatest signal-to-noise ratio for the sum. The signal used for this optimization is the rate from the HEU source at the bottom of the cargo container with a full load of wood cargo. The noise is the square root of the total background rate with the same full load of wood cargo. The signal-to-noise ratio is plotted in Figure 18 as a function of the number of summed detectors starting from the center detector. The inset shows a detail of the curve near its maximum, the section corresponding to the 15 to 25 nearest detectors.
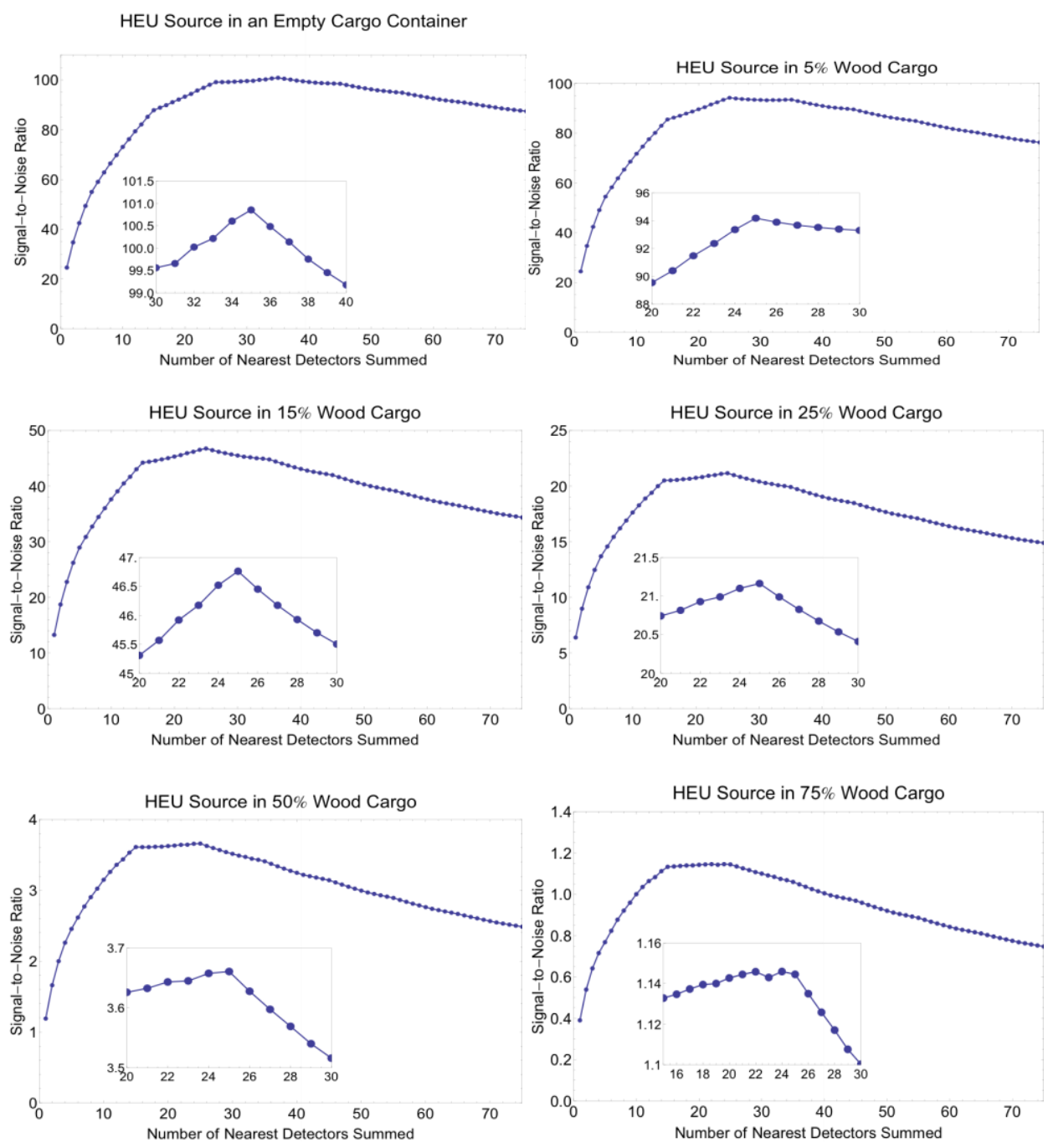


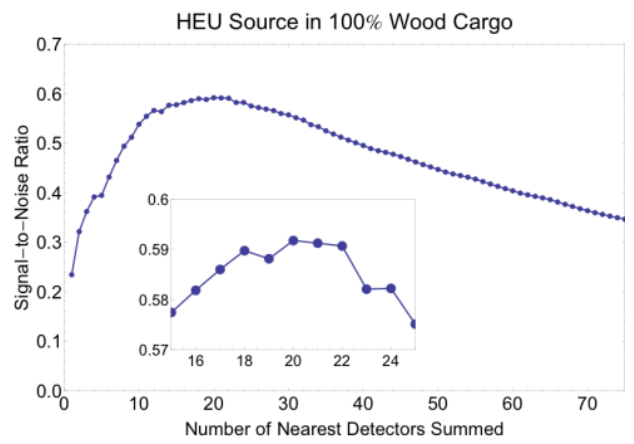

Figure 18. SNR for the summation of detector elements.

The validated MCNP model described in the preceding section, and example shown in Figure 13, is used for optimization of detector subset. For each depth of cargo two MCNP simulations were performed: one with the HEU source and one with the soil source (as background). The background data was simulated by two modeled sources, one representing the direct gamma-ray contribution from the background, and the other representing the skyshine component from the surrounding air. The first (and dominant) source of radiation was similar to an earlier MCNP "terrestrial background" disk source emitting around 2.1 gamma rays $/\left(\mathrm{cm}^{2} / \mathrm{s}\right)$ from points on the ground [19]. Data from all 32 crystals were simulated (Figure 19). Sources in several locations are shown in Figure 19 and observable as prominent elevated regions in the net counts.
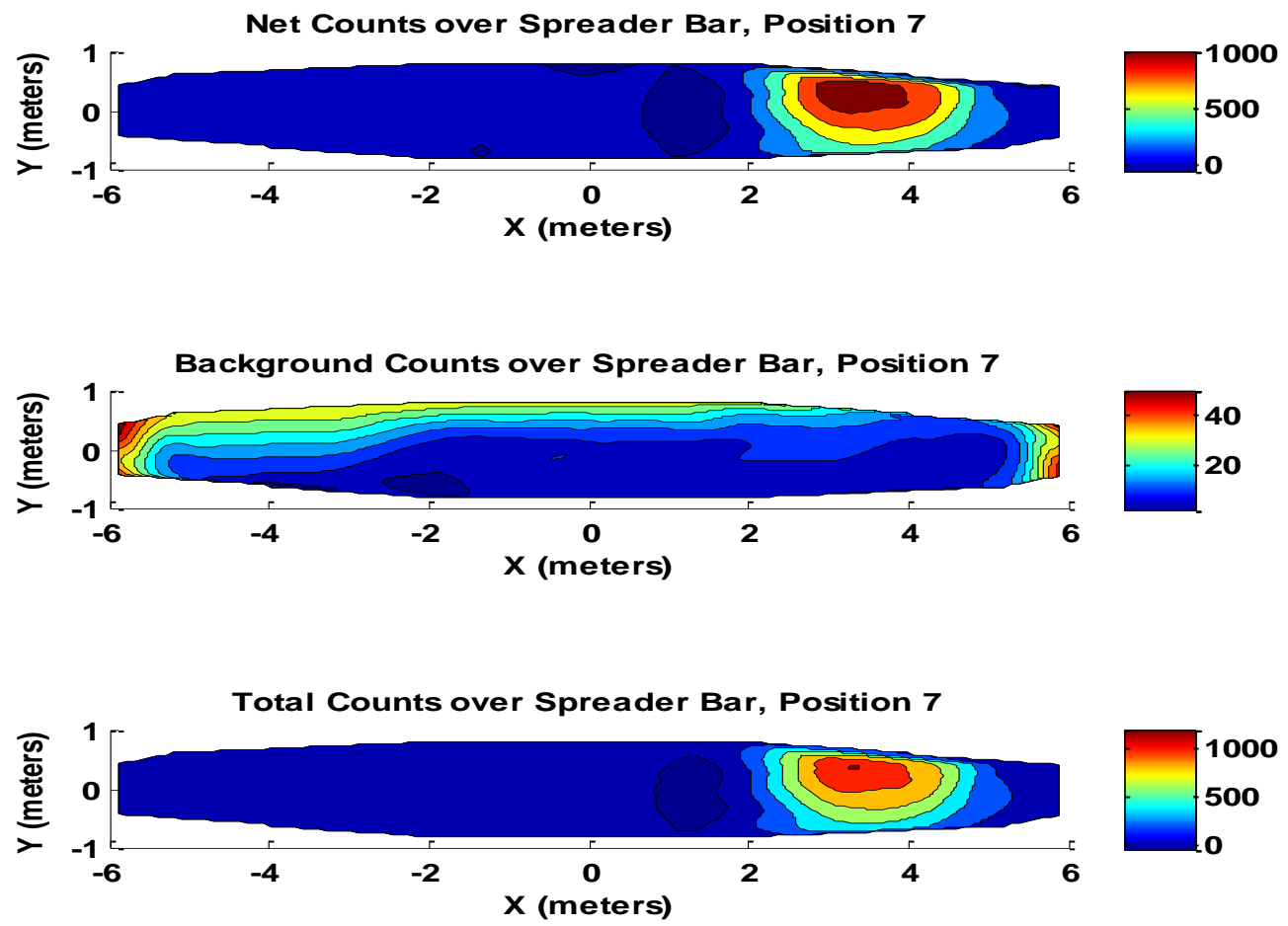

Figure 19. (a) Source, Background and total Counts for a source in position 7, depth=0\%. 

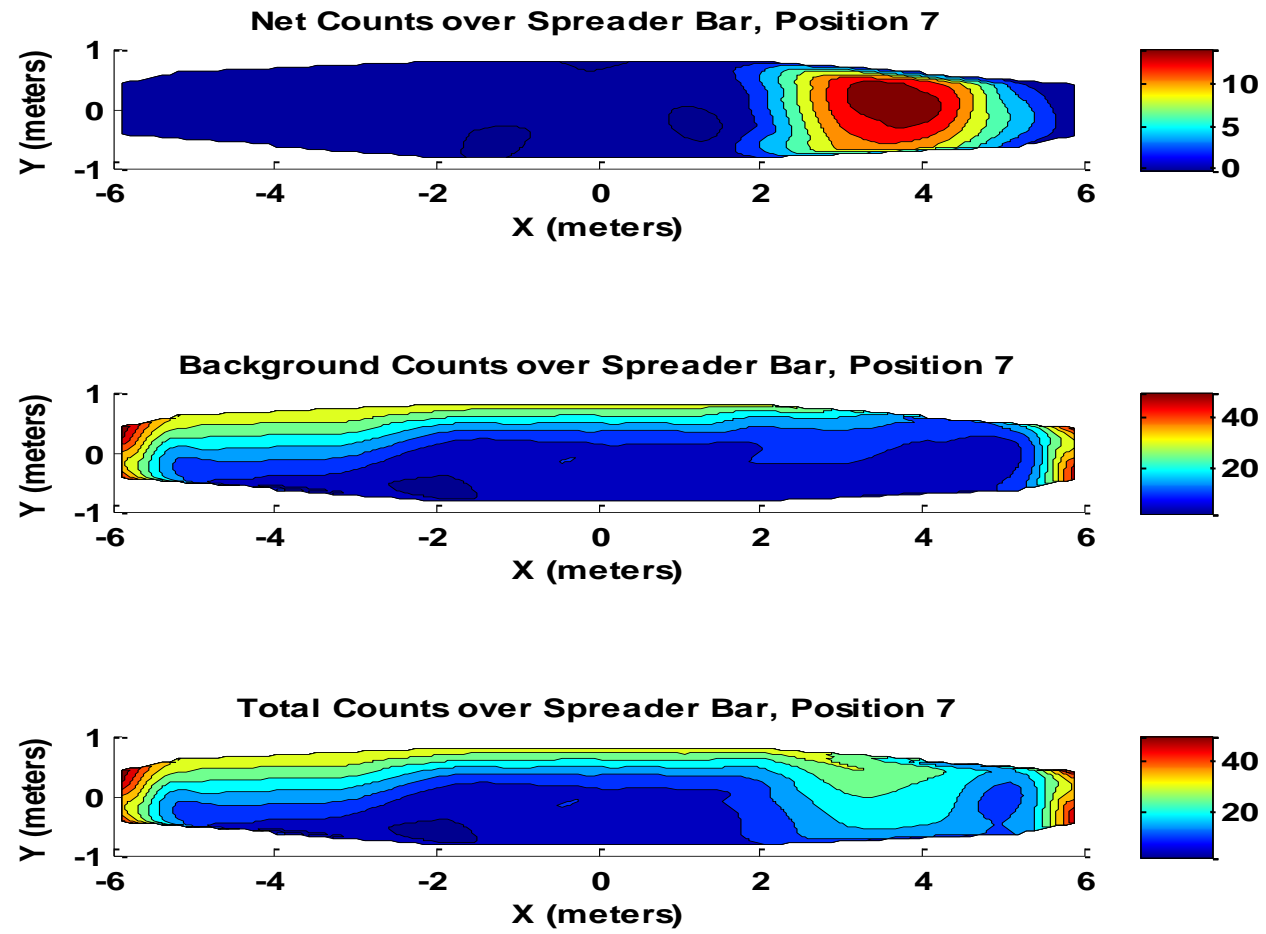

Figure 20. (b) Source, Background and total Counts for a source in position 7, depth=50\% .
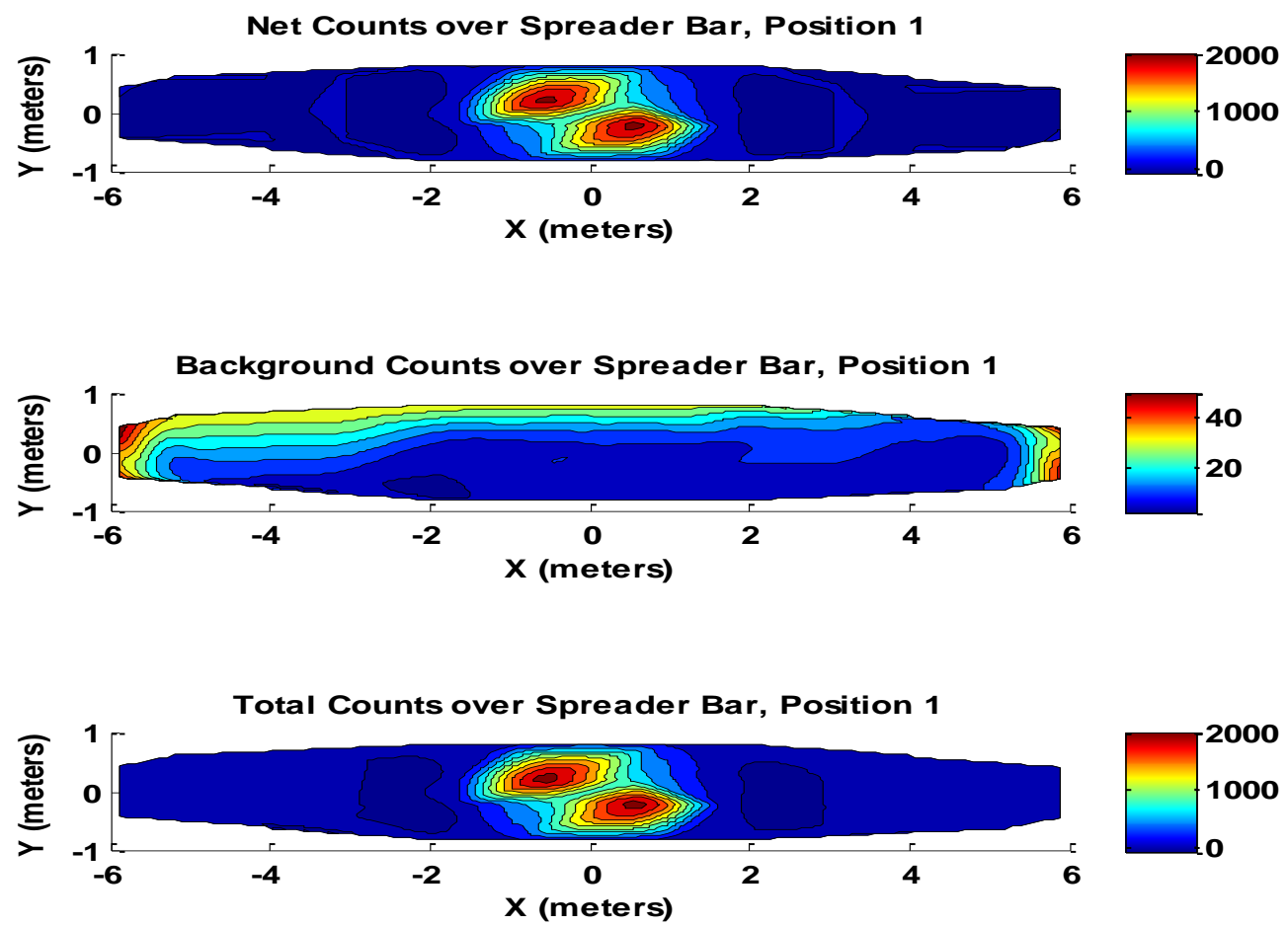

Figure 20. (c) Source, Background and total Counts for a source in position 1, depth=0. 

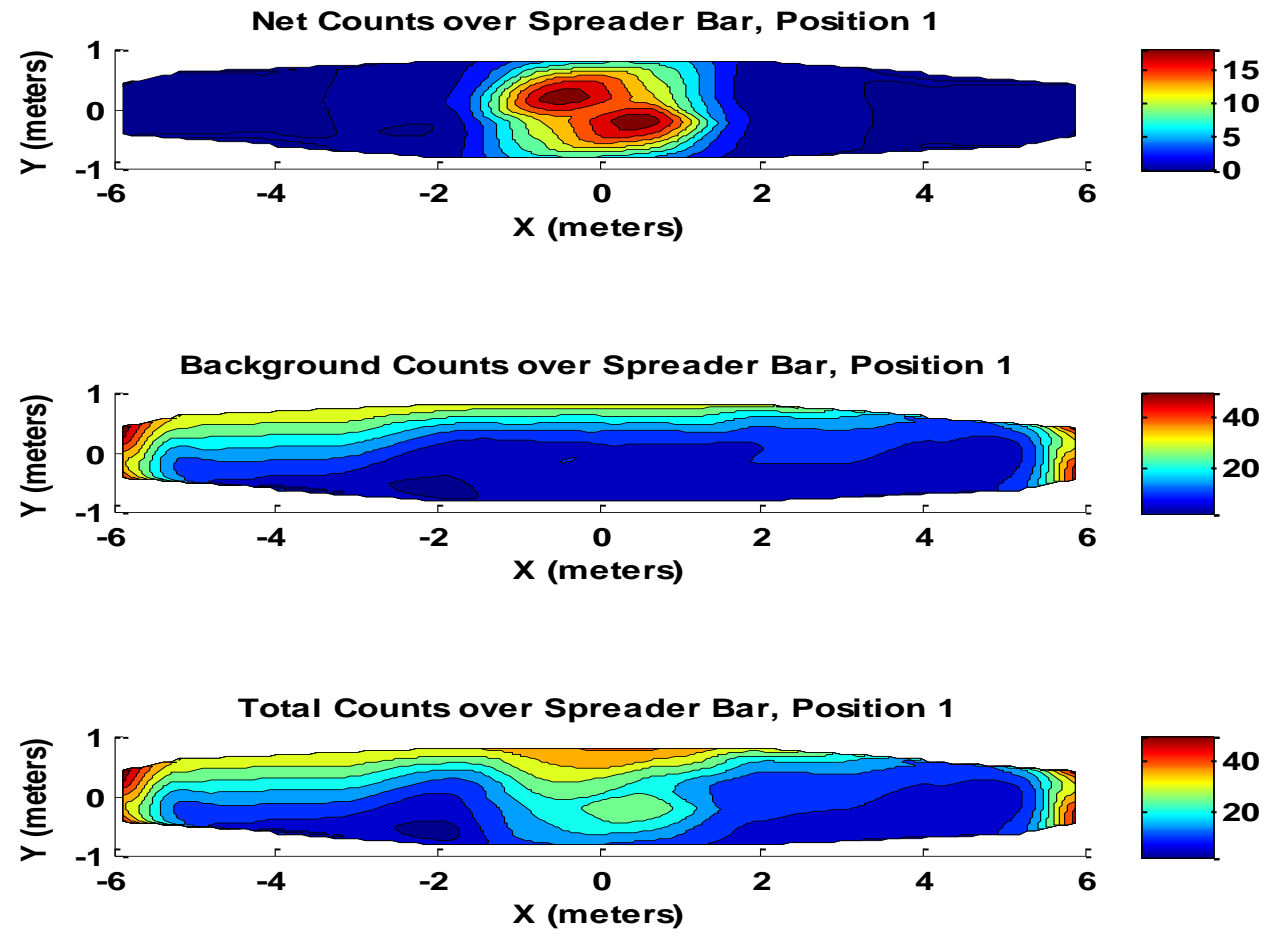

Figure 20. (d) Source, Background and total Counts for a source in position 1, depth=50\%.

The exact location of individual detectors in the sparser detector model makes a great deal of difference to the intensity map. While this is not expected to hurt the results gained from summing results over the optimal detector subset (by the arguments made earlier), it may pose a challenge to source localization with this method, as the source cannot be expected to give a consistent "count-rate pattern" given the scarcity of detector elements.

For each detector element, the signal-to-noise ratio for groups of detectors is calculated as the ratio of the total signal counts in the group of detectors to the square root of the total background counts in the group of detectors. An optimal choice of detector subset is expected to include the center detector (the one most directly above the HEU source) and some number of detectors that are closest to this center detector, and approaches which automatically choose this optimal subset are explored in the following section. The signal-to-noise ratio is calculated as a function of the number of detectors for various thicknesses of wood cargo, as a percent of the maximum thickness. In each case, the optimum number of detectors, which maximizes the signal-to-noise ratio, is plotted against the thickness of wood cargo as a fraction of a full cargo load (Figure 20). The curve drops off sharply as thicker cargo is introduced, and in general suggests fewer detectors as cargo moves toward $100 \%$. While further evaluation of different thicknesses may add structure to this curve, the most important result is that the optimal detector subset varies as cargo and source configuration varies. 
PNNL-21948

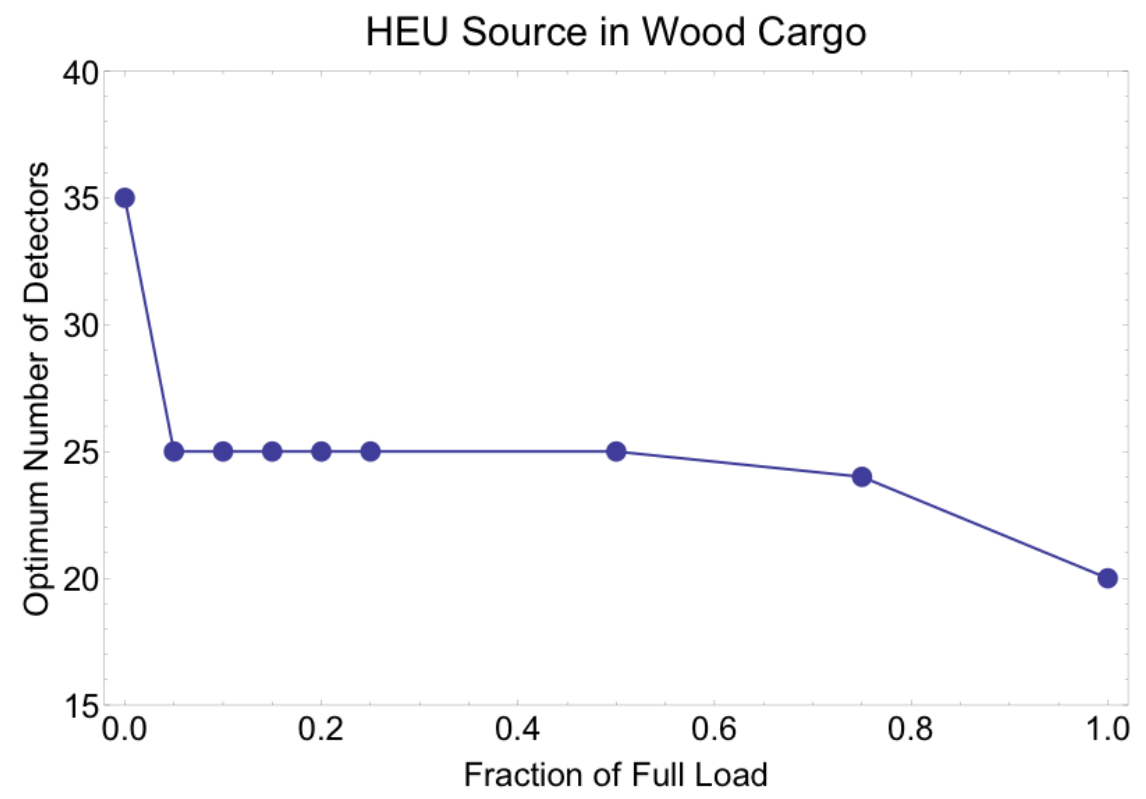

Figure 20. Optimum detectors in subset vs. cargo load with source at container bottom.

Another approach is to look for the optimum size for a maximum load of wood cargo, but allow the source to be located at different depths from the top in the cargo. This arrangement is the same as shown in the figures above, except that the wood cargo is always $223 \mathrm{~cm}$ thick, and the source is placed at different depths below the top of the wood cargo. To illustrate the falloff of increasing performance with a larger and larger detector subset, the signal-to-noise ratio is plotted against the number of detectors for various thicknesses of wood cargo, as a percent of the maximum thickness. In each case the inset shows the area of the curve around the maximum signal-to-noise ratio (Figure 21). The signal-to-noise ratio is plotted against the number of detectors for various depths in the wood cargo, as a percent of the maximum thickness. In each case the inset shows the area of the curve around the maximum signal-to-noise ratio.
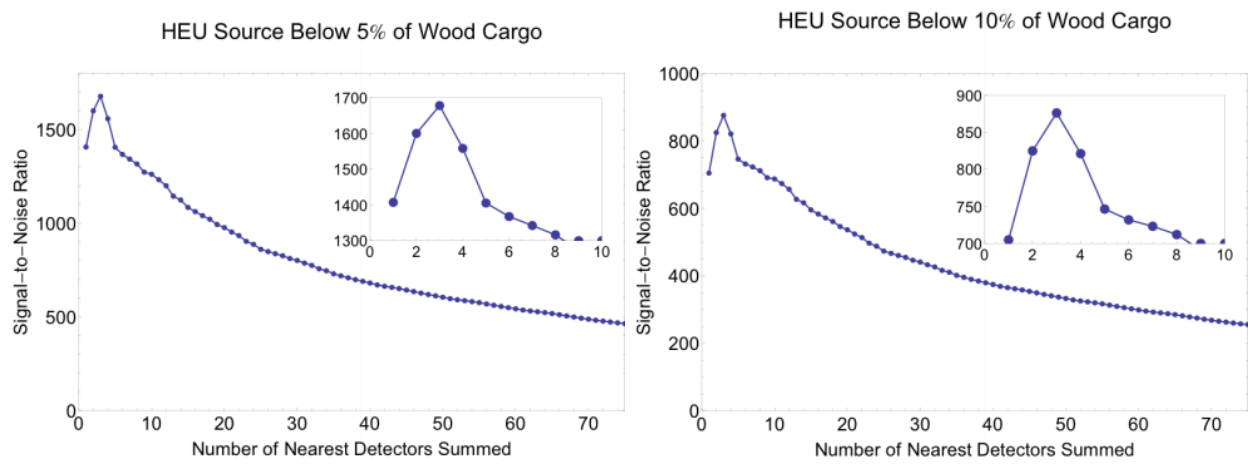
PNNL-21948
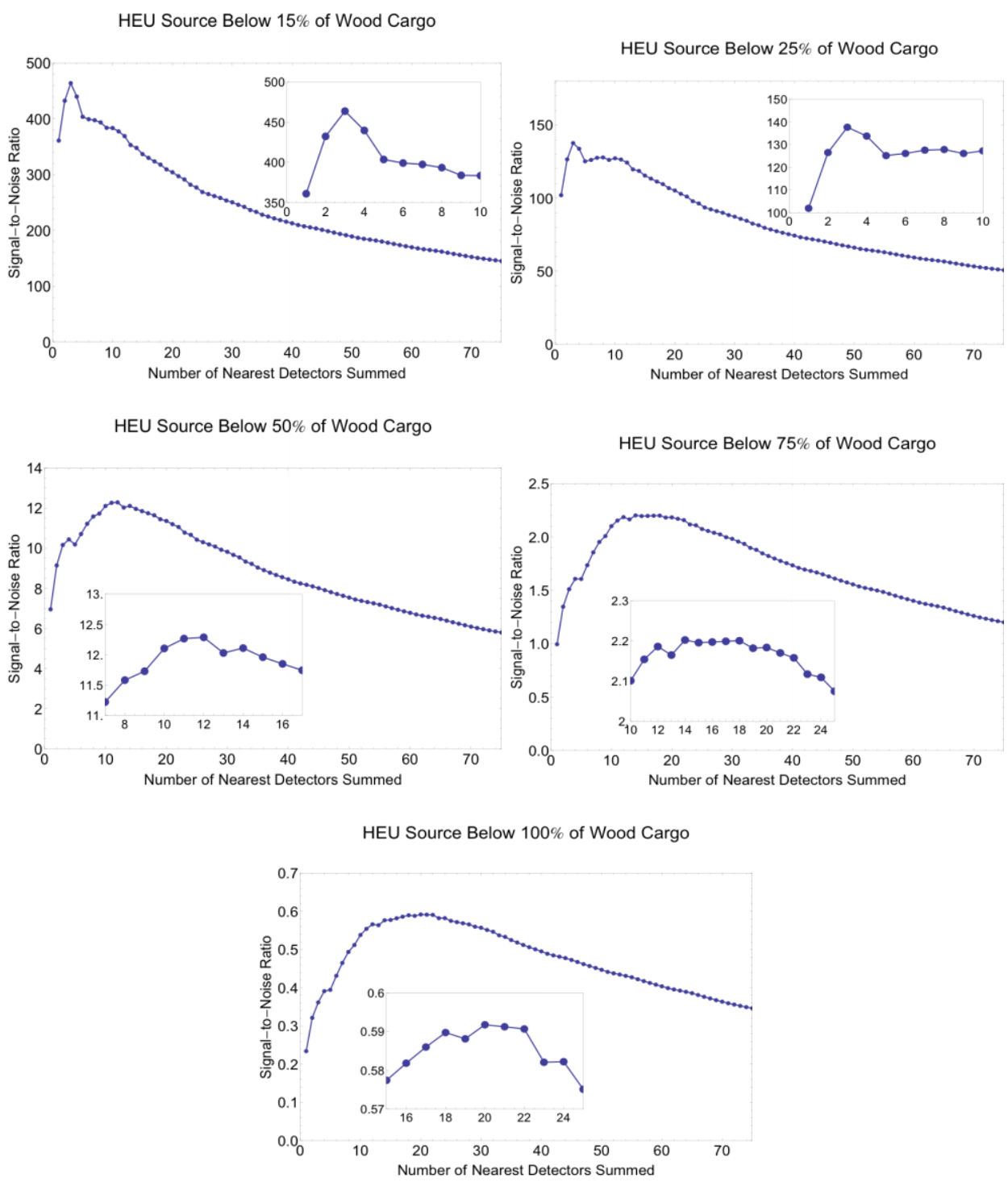

Figure 21. Signal-to-noise ratio for the summation of detector elements.

Again, the optimum number of detectors, which maximizes the signal-to-noise ratio, is plotted against the thickness of wood cargo as a fraction of the maximum thickness in Figure 22. 
PNNL-21948

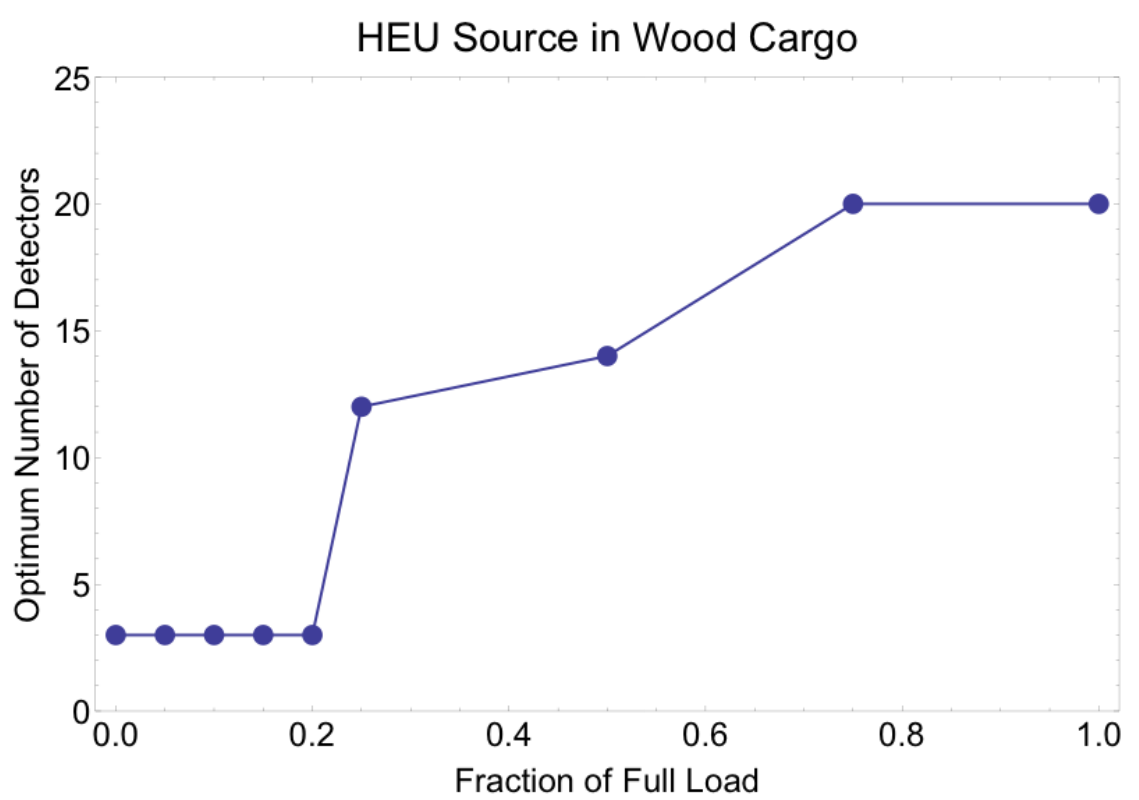

Figure 22. Optimum detectors in subset versus cargo load with source at different depths in a fully loaded container.

In this case the dependence on amount of cargo above the source is very different than when the source is always on the bottom since the source becomes much closer to the center detector as the amount of cargo above it decreases. Up to $20 \%$ of the cargo depth, when the source is close to the center detector, the optimum number of detectors is only three. As the source moves down to the bottom of the container, the optimum number of detectors approaches 20 , in agreement with the analysis above for the source always on the bottom of the container.

This varying number of detectors for summation poses a problem for the subset algorithm, as the cargo depth will not be known during measurement by the SBRD, and no single number of detectors optimizes SNR across all cases. Therefore, the algorithm would have to use cues from the measured radiation data to provide an optimal subset.

\subsubsection{Optimal Detector Subset - Iterative Methods}

A varying optimal subset of detectors was observed in each measurement scenario, leading to an inherent difficulty in assigning an optimal set before measurements are made. To approach this issue, an iterative method was applied, intended to be applied at the time of measurement, in order to determine the best detector subset to use. This method will iteratively attempt several subsets, in keeping with the assumptions and constraints of this section, in order to determine the optimal one for each measurement.

The SNR of each detector element is first considered when the HEU source is placed near the middle (Figure 23). The benchmark of 25 included crystals does not require the same SNR cutoff for each source location, which makes deciding on an explicit (e.g. single and unchanging) SNR cutoff as a selector algorithm ineffective. Instead, even crystals with substantial SNR should be excluded from the measurements with low shielding, while the deepest shielding requires the addition of crystals with very low SNR, as made obvious by the examination of individual detector SNR under various conditions. 
PNNL-21948

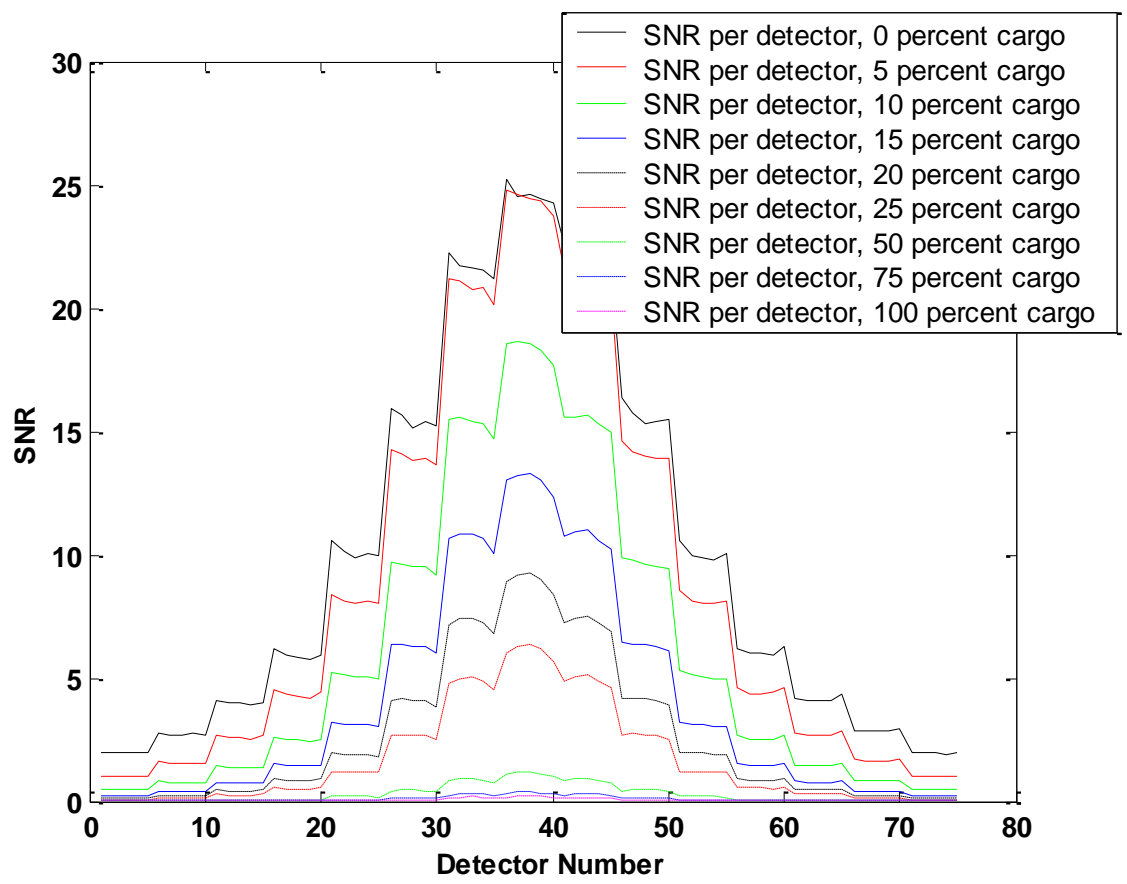

Figure 23. Detector number versus signal-to-noise ratio.

In general, assuming the background to be roughly equal across detector crystals (or only slowly varying as shown in central plots of Figure 19), we could write the summation of SNR figures for several detectors as:

$$
\sum_{i=1}^{N} \frac{S_{i}}{\sqrt{B}}=\frac{1}{\sqrt{B}} \sum_{i=1}^{N} S_{i}=\frac{\sqrt{N}}{\sqrt{N B}} \sum_{i=1}^{N} S_{i}=\sqrt{N} \frac{\sum_{i=1}^{N} S_{i}}{\sqrt{N B}}=\sqrt{N} \frac{\sum_{i=1}^{N} S_{i}}{\sqrt{\sum_{i=1}^{N} B}}
$$

Where $S_{\mathrm{i}}$ is the source counts in detector $\mathrm{i}$ and the $\mathrm{B}$ is the background rate.

A "compactness criterion" is also added to the optimal detector subset algorithm. It is expected that sources of interest will be compact, and therefore the set of detectors reasonable to use will represent a simply connected "spot" on the surface of the spreader bar. A radially symmetric source in a homogeneous medium produces circular contours (or portions thereof) of constant flux on a face of the container. This suggests the use of circles to define regions of maximal SNR around the point on the surface nearest the source.

Pfund et al. [20] studied the similar problem of finding the optimal measurement time for continuous measurements in terms of maximizing SNR, where a source and detector are in relative (linear) motion. Conversely this can be thought of as finding the distance from the closest approach to the source within which measurements should be summed to maximize SNR. This is effectively a continuous 1-D version of the SNR optimization problem as stated above. In the 1-D case, a theoretical maximum distance exists. One can easily reason that there is an optimal subset of a discrete array of detectors. 
The basic notion of finding maximal total SNR for a set of detectors within a closed region seems sound, and the circle is used as an appropriate choice for roughly homogeneously spaced detectors.

The algorithms attempts to determine which circle (in terms of location and area) optimizes the overall SNR by centering this "spot" on the detector with the highest individual SNR and find the radius by an iterative method. However, this neither accounts for the case where there is no source (and thus the choice of maximal detector is incidental) or the case where the source is between detectors or otherwise difficult to localize.

Another approach would be to use a slightly larger "initial circle" - for example, an annulus around 1 meter in radius, and then determine which location for this circle maximizes SNR. Thereafter, the center and radius of the circle (or just the radius) can be optimized with simple nonlinear fitting methods. Additionally, detectors at the edge of this contour can be included or excluded from the measurement by examining their individual contribution to the total SNR. Results from these approaches are shown in Figure 24.
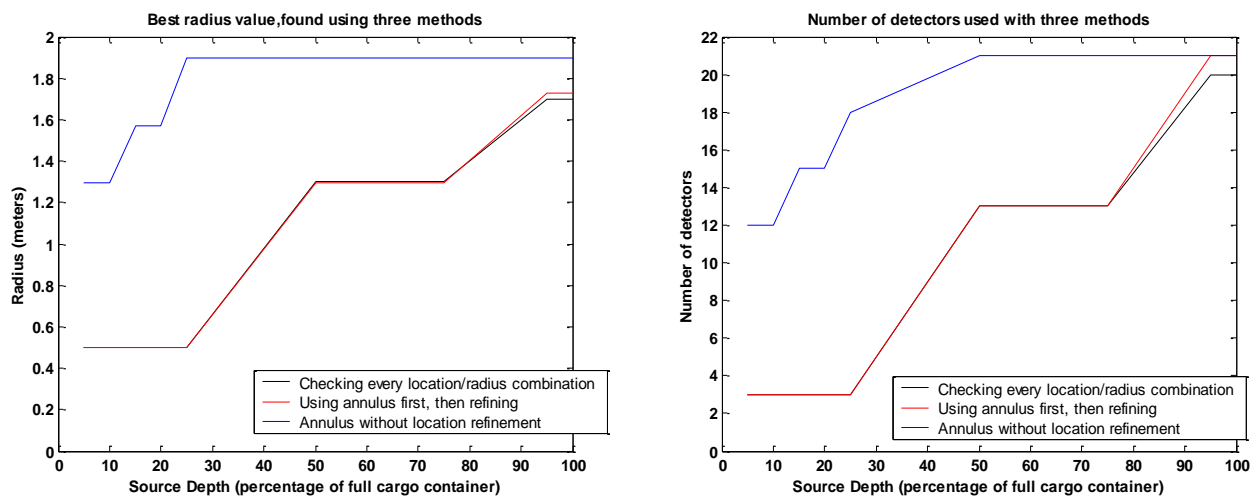

Figure 24. Plots of the results of several methods used for selecting optimal detector subset.

The "checking every location" and "annulus with refinement" methods produce similar results. The optimal number of detectors chosen by the annulus methods also closely matches the optimal number chosen by summation as performed earlier. For the sake of operation with limited computational resources, the "annulus with refinement" method is chosen as an efficient metric for the production of data subsets. This method may also allow for an estimate of source location at all depths.

As an additional consideration, it is not completely known if the optimal subset for determining gross-count SNR is also the best for other algorithms, or if maximizing the gross-count SNR does indeed maximize sensitivity, or instead tends to exaggerate the measured SNR of background-only measurements. Every algorithm - e.g., gross counting, energy windowing, anomaly detection, discriminant analysis, peak-fitting, template matching - relies on whatever information is available in some subset (or all) of the energy range of the spectrum measured at each sensor. Let E represent the combination of all energy regions of interest for a given algorithm. The SNR at sensor $i$ for the algorithm may be defined as

$$
\frac{S_{i}(E)}{\sqrt{B(E)}} .
$$


Let $S_{0}(E)$ represent the signal that would be measured at a sensor directly above the source (whether a sensor is actually in that position or not). Let $\mathbf{r}_{i}$ be the distance between sensor $i$ and the source. Considering only the loss of photons to absorption and scatter, we may posit an attenuation model

$$
S_{i}(E)=S_{0}(E) \exp \left[-\mu(E) \mathbf{r}_{i}\right] f\left(\mathbf{r}_{i}\right),
$$

where $f$ is some function of the distance.

Summing measurements over some set of sensors $i=1, \ldots, n$ the total SNR is

$$
\frac{\sum_{i=1}^{n} S_{i}(E)}{\sqrt{n B(E)}}=\frac{\sum_{i=1}^{n} S_{0}(E) \exp \left[-\mu(E) \mathbf{r}_{i}\right] f\left(\mathbf{r}_{i}\right)}{\sqrt{n B(E)}} .
$$

It is clear from this form that maximizing this SNR over the number of sensors $(n)$ does not depend on background or strength of the source $\left(S_{0} / \sqrt{B}\right.$ scales out as a factor independent of $n$ ). The optimal number of sensors depends on distance from the source (or equivalently, distance from the sensor nearest the source). It also depends on the algorithm through the choice of energy regions of interest, as is evident in that dependence coupled to the distance in the exponential. If the dependence of attenuation on energy is relatively weak, then this dependence may be ignored and we may say that optimal number of sensors is independent of algorithm. Strong differential attenuation could definitely impact the optimal number of sensors, but should not affect the choice of circular regions as a basis for maximizing SNR.

In order to verify optimality of subsets chosen by this method, we first expand the data set used to include several additional location sets in the cargo container (Figure 25). Data (and gross-count digests) are produced for each location, with associated backgrounds. The aforementioned adaptive algorithm for optimal subset finding is produced as mentioned above and run over this expanded set of data (Figure 26).

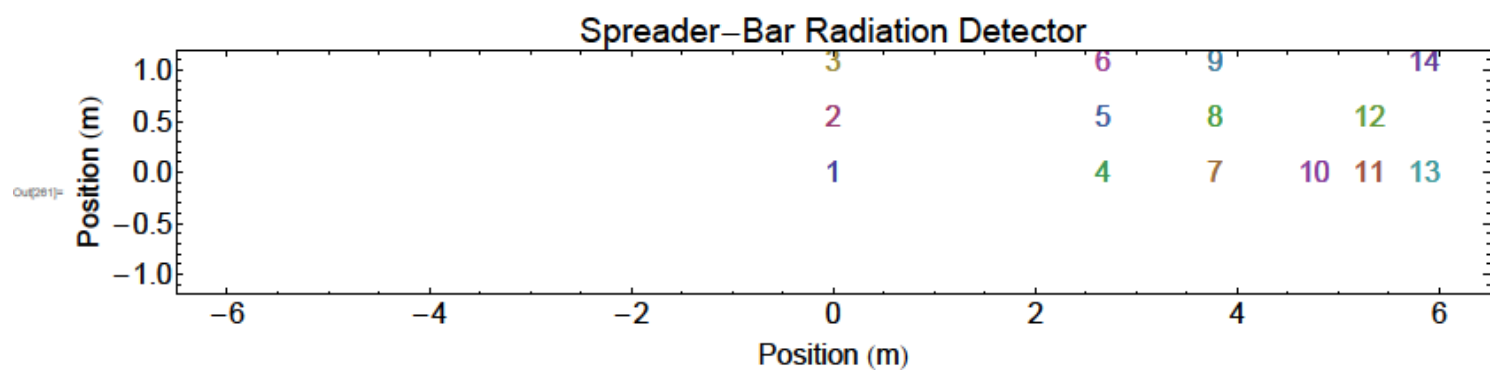

Figure 25. Enumerated source locations used for the simulation. 

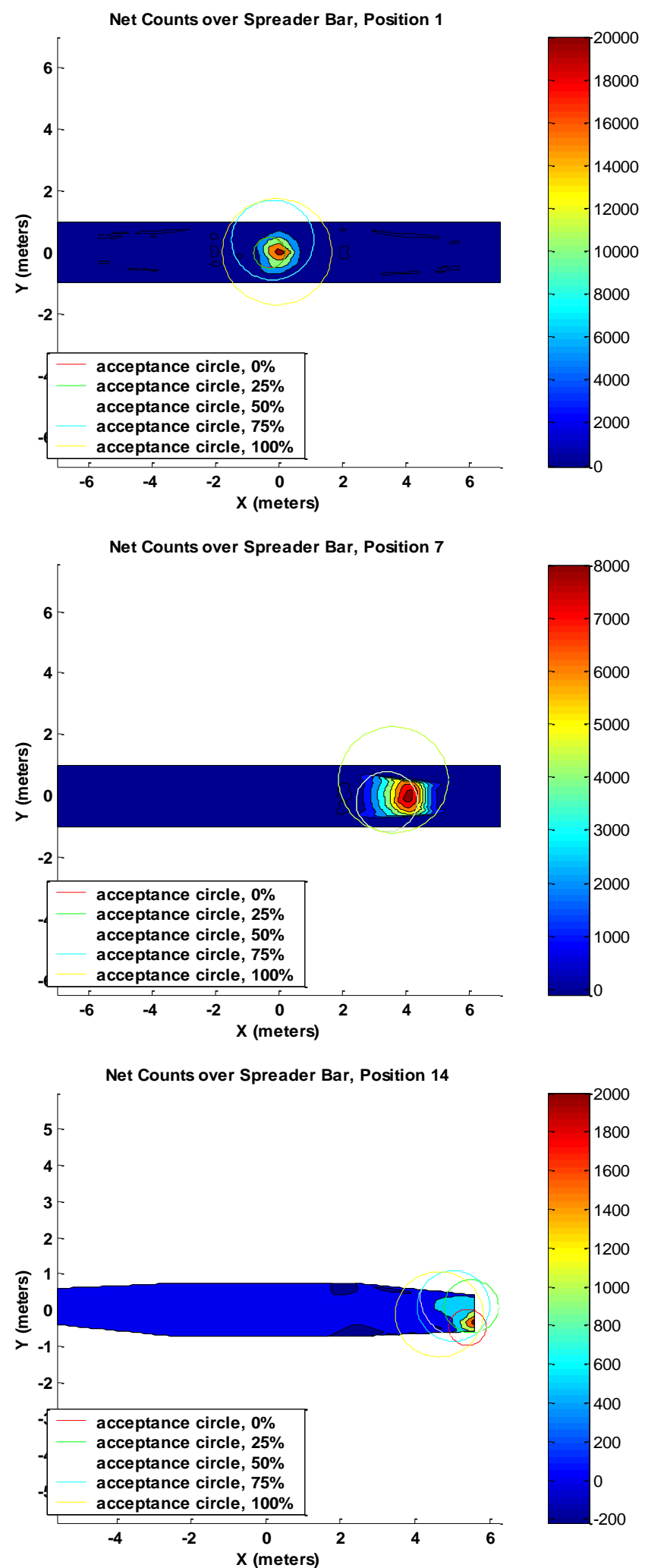

Figure 26. Optimal detector subsets for SNR maximization, as chosen by annulus algorithm, several cargo depths (0 to 100\%). 
In addition, the $\mathrm{x}, \mathrm{y}$, and radius values chosen are shown superimposed on the map of count rates for $0 \%$ cargo depth. The algorithm generally selects the correct location, with minor excursions about that value. The inclusion or exclusion of detectors near the edge of the acceptance region should not greatly change the overall SNR.

The above approach will then be applied to the specific problem of gross-count sensitivity in a detection context, to show optimality for real detection. The "optimal detector subset" choice algorithm just created will also be applied to background-only data to investigate the issue of false positives. The combined SNR obtained in this way will be compared with the combined SNR from a similarly sized random selection of crystals, with the understanding that actually alarming on source data will mean optimizing the response of a detection algorithm as compared to the response to background data. Therefore, any increase to SNR obtained by using a down-selection of crystals must be compared with the expected increase in SNR with "no-source" runs when using that same method. It is expected that, for example, a method where all the highest-count detectors are summed regardless of location would produce a higher measured SNR when presented with background data than a method using compactness and connectedness of the detector subset. It is expected that the SNR from a connected detector set will not be far in excess of the SNR from a random set, but this must be verified, and this "background" SNR will also be used for a lower threshold when determining alarms from data.

In order to demonstrate the above argument, the data are produced with and without a real source present. Many realizations are made of each scenario by adding the Poisson noise appropriate to a 30-second measurement (Figure 27). Likewise, the "no source" data are produced by adding stochastic noise once more to the background data and using the residual error in each energy bin as a source term. The data in each case for the "random detectors" SNR was found by selecting the same number of detectors at random as are used for the corresponding "best spot" method.
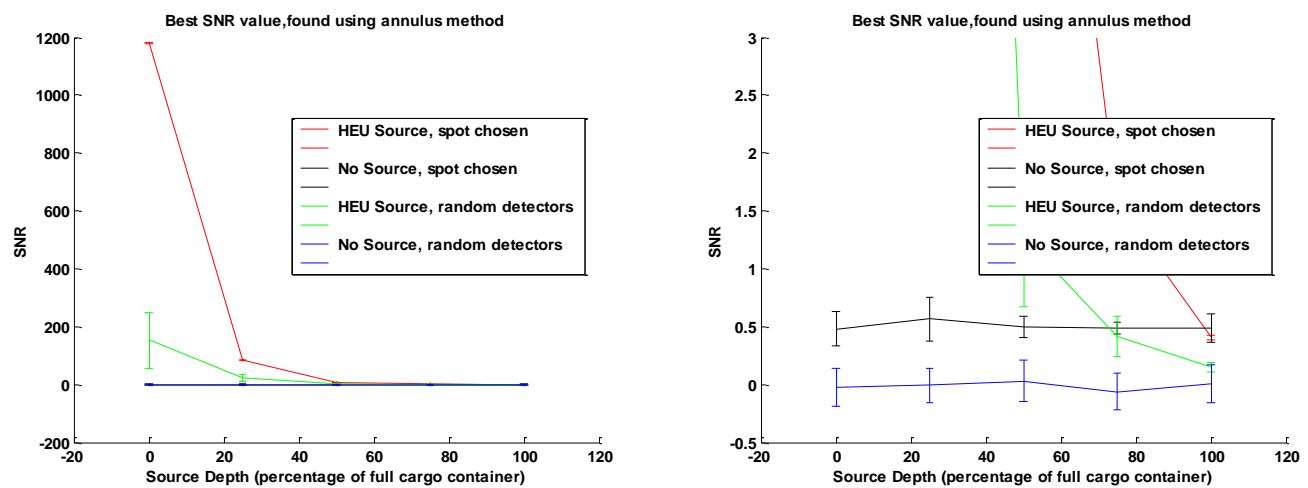

Figure 27. Results for best detector subset, with and without HEU test source (source at loaction 7 of Figure 25). Both plots are of the same data, with the right plot having a different y-axis range to show low level detail.

As expected, the "best values" become close to random when the spot for detection is fit to a situation with no actual source. The error bars in this situation become very large, and no structure is readily discernible. Likewise, the SNR as measured with a group of random detectors and no source hovers around zero. However, there is some enhancement to this value when a spot is chosen with the above methods but no source is present. As would be hoped, this enhancement is small, averaging around 0.5 sigma; far lower than the expected value for gross count detection against the background. This small difference to the SNR algorithm is not expected to significantly affect the detection capability of the system, and will represent a similarly small alteration to the detection threshold used for an 
algorithm when results are analyzed in the field. This alteration will be accounted for by the full comparison of algorithms, and represents an overall benefit to the detection apparatus. Likewise, these results show a far superior result from the annulus method than a random selection of crystals would allow, given a present source.

\subsection{Data Production}

Optimal subsets for detection are produced in keeping with these simulated data sets, by maximizing the gross-count SNR value for a given subset. For each source location and depth, the list of detectors to use is kept as a binary vector, and stored in a file (locations shown in Figure 28). Note that this is performed for a substantial multiplication of the source counts to ensure proper subset selection. At a depth of zero, a single source's emissions are used, at $25 \%$ depth this rises to 25 sources, 50 at 50\% depth, and so on. This is intended to be beneath the expected MDQ for these depths, for most detection methods, but does not affect the choice of optimal subsets at these levels.

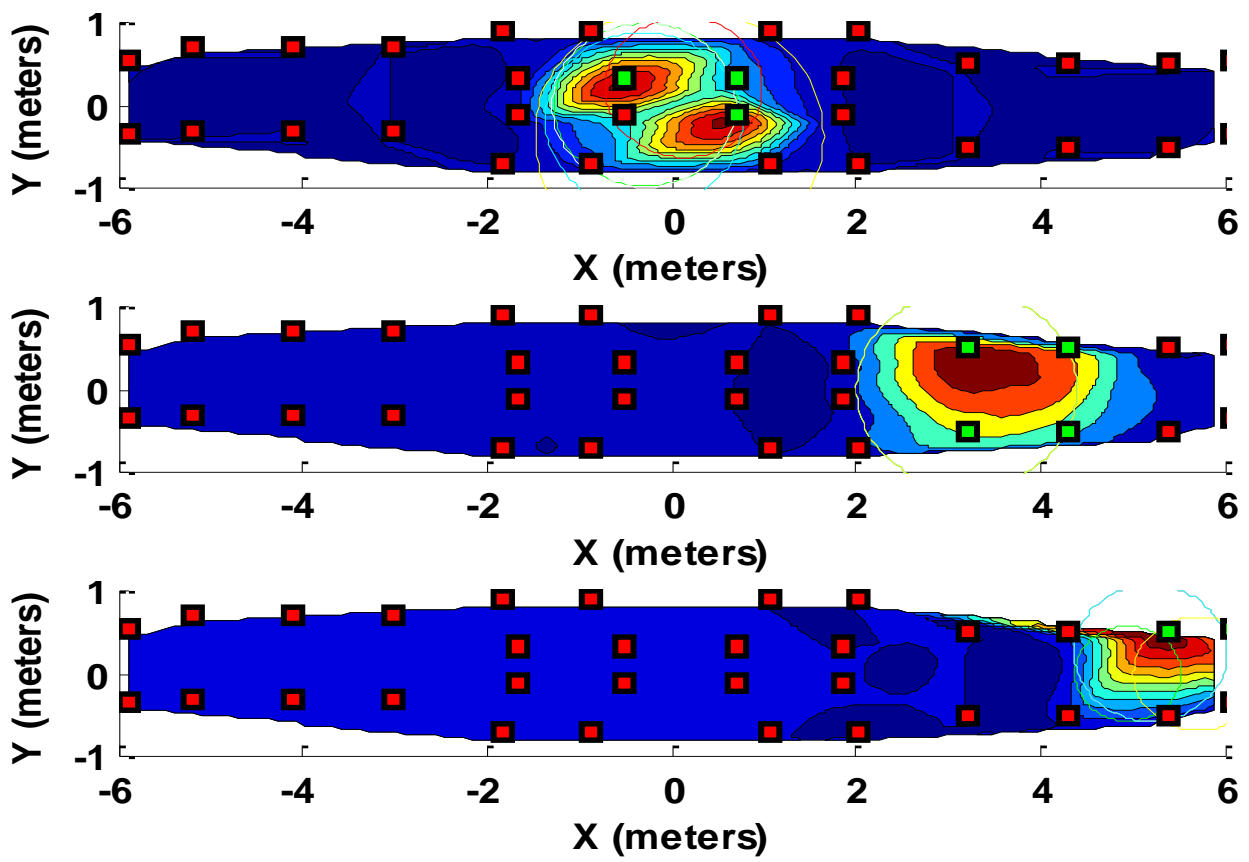

Figure 28. Overall Count Rate and Optimal Detector Subsets, included detectors for depth=0 shown in green. Top: Source Position 1, Middle: Source Position 7, Bottom: Source Position 14.

A second stage is made to generate data according to these subsets. Specific details of this generation stage are included in Appendix A.

\subsubsection{Detection Algorithms}

Several algorithms are chosen to attempt a variety of approaches, in keeping with the various approaches used for source detection and identification in fielded detection systems, as well as recent advancements in these approaches [21]. These are summarized briefly here:

Gross Counting: The gross-counting algorithm is one of the simplest methods of source detection and is used in many deployed detector schemes. In this algorithm, the number of counts above the 
background is computed and compared with a threshold. The figure of merit $\mathrm{F}$ is generally calculated:

$$
\begin{aligned}
& C=B+F \sqrt{B} \text { or } \\
& F=(C-B) / \sqrt{B}
\end{aligned}
$$

where $C$ is the total counts and $B$ is the background counts. Gross counting is thought to be an effective "baseline" solution as its performance will not be significantly changed by anything other than attenuation by cargo materials.

Energy Distance: Previous work has shown improved results in some cases of source detection with the use of "energy distance" anomaly detection algorithms [15]. In this approach, the available spectrum is first binned into coarse energy bins (eight bins are used for this investigation). These bins are treated as an eight-dimensional vector and normalized. Then the "opening angle" between background and source spectra is found by taking the dot product between them. This opening angle is the figure of merit returned from this algorithm.

Peak Finding: The peaks present in the spectroscopic data can be "picked out" with a variety of methods and then compared with a catalog of known source emission lines to produce a list of the isotopes present in the measured spectrum. The peak-finding algorithm used for this work first subtracts background and then takes the convolution integral of the data with a Gaussian peak of width matching the detector resolution. The resulting function over energy is compared with a threshold to generate peaks and these peaks are compared with a limited catalog of sources to determine source detection. In this case, a detection of HEU (that is, a detection of the relevant emission lines from uranium-235) is considered to constitute a positive detection, while any other isotopes are ignored.

GADRAS: In the template-matching technique used by the Gamma Detector Response and Analysis Software (GADRAS [17]), the spectrum to be analyzed is compared with a library of templates. When a "good" match is found, the spectrum can be identified as that of the isotope used to generate the template. The template library contains the measured or calculated spectra of most isotopes as well as some background spectra.

Template matching is well suited to the identification of unshielded radiation sources; multiple regressions can also be applied to identify combinations of radionuclides. Template matching can be performed quickly with limited computational resources, a desirable feature for use in handheld radioactive isotope identifiers. The GADRAS template-matching code was used in this work for identification of the HEU source. In this case, the presence of uranium in the list of detected sources is used as a positive indicator of detection, meaning that the figure of merit for this use of GADRAS is effectively binary. While false alarms may be present in the form of declarations of other sources not present in the data, there is no trivial way to set a threshold on detection using the GADRAS algorithm, and the probability of detection is therefore reported irrespective of false detections.

Limited GADRAS: GADRAS is employed in one other fashion in this work. In this technique, only the natural isotopes expected in background were included in the GADRAS template library. The presence of an additional source will tend to make the "goodness of fit" progressively worse with intensity. Thus, the goodness of fit $\left(\mathrm{Chi}^{2}\right)$ is used as the figure of merit for this use of GADRAS, rendering it an anomaly-detecting algorithm. 
KUT: In this approach, the Potassium, Thorium, and Uranium components of natural background are simulated at equilibrium, in a deeply shielded configuration. These components form the basis of terrestrial background models for Monte Carlo radiation transport simulations performed at PNNL. Each component is simulated with MCNP as incident on the detectors in the spreader bar model. The resultant spectra are used as the three components for a linear fit to the observed spectrum, and the sum of square errors is used as an anomaly metric.

QuID: A new algorithm developed at PNNL, called QuID for "quick ID", does not find the peaks explicitly, but rather performs a general linear least squares fit of a series of isotopic models against the spectrum [22-23,23]. The spectrum is first rebinned such that its peaks have a full width at half maximum that is constant. This simplifies the computations that follow. A set of Gaussian models representing the isotopes in the library are then created, and the models and gamma ray spectrum are filtered using a zero-area square wave filter (SWF). Filtering the spectrum enhances the peak signatures and minimizes the baseline, thus preventing the need to subtract it out or model it accurately. The models are then fit in linear combination against the filtered spectrum using an iterative non-negative linear least squares technique. Output of the fit is the relative source strength of each model and its uncertainty. The ratio of these two quantities is compared against a single threshold to determine if the isotope is present in the spectrum.

\subsubsection{Data Analysis}

Results from selected algorithms are shown, to show the algorithm results for each source depth and strength (Figure 29). These results are plotted with respect to source strength and depth: 
PNNL-21948
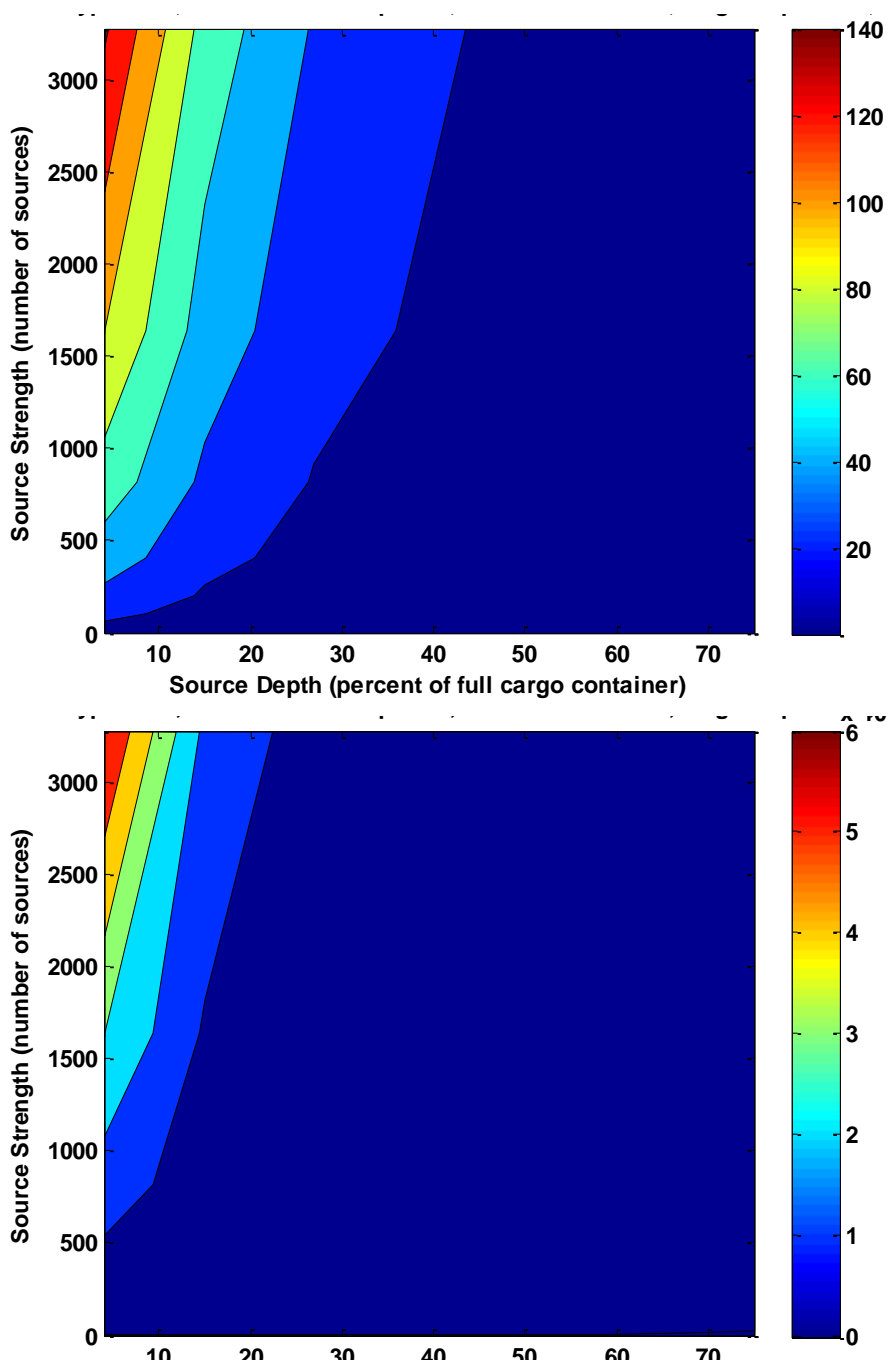

Source Depth (percent of full cargo container)

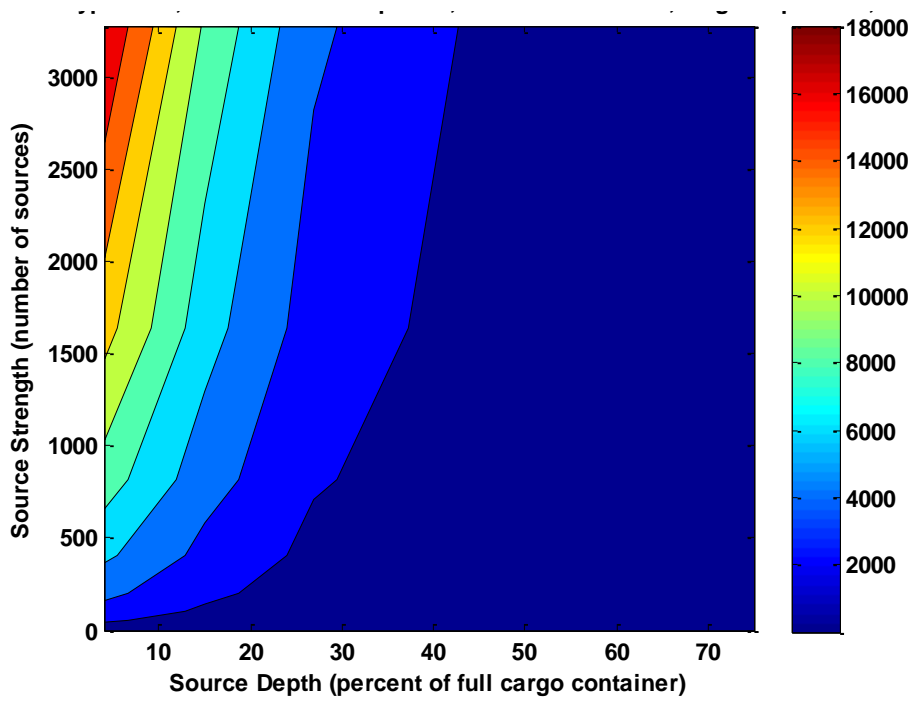


Figure 29. Average Algorithm data for Optimal Detector Subsets, for varying source depth, strength (Central source location shown). Top: Energy Distance Algorithm. Middle: Gross Count Algorithm. Bottom: KUT Algorithm.

Several thresholds for detection must be produced for each algorithm involved. A NORM (i.e., assuming that NORM sources must be avoided) and non-NORM (i.e., assuming that NORM will not be considered) threshold must be produced for each algorithm to provide alarm results. These thresholds will be produced from the algorithm results.

For each algorithm, the raw data are parsed, and a threshold for detection is set. Two such thresholds are made, either considering NORM sources as a component of background or not. In either case, the threshold is set to exclude all the results from these backgrounds in order to provide alarms. Two figures of merit (Algorithm source FOM, Algorithm false alarm FOM) are also produced by analyzing the simulated data with each algorithm. In a real scenario, the source is not known beforehand. Therefore, we set these thresholds to exclude the "false" FOM (the rate of positive detection from scenarios with no actual sources). Thresholds for detection are estimated by excluding only false identifications that occur in background or NORM cases. The thresholds based on this reasoning are saved in a file containing the thresholds to use for each algorithm.

To produce MDQ figures, we apply each algorithm to all the background files and produce data digests containing values of cargo depth, iteration number, and figure of merit. We also apply the algorithms to our source data set to produce another file with the values of cargo depth, source rate, iteration number, and figure of merit. We declare the acceptable false alarm probability to be about $1 / 1000$ and determine an appropriate alarm threshold that gives this for each algorithm, for any part of the data set not containing any source term (i.e., taking the statistical limit of algorithm performance).

These results are post-processed by comparing the alarm algorithms for each algorithm to the figures of merit for all the source runs. The number of source runs exceeding the thresholds are combined to produce plots of source emissions versus probability of detection for each algorithm and depth. After this, for each source depth, the source emission rate necessary to produce a probability of detection of 95\% is found by interpolation of the data and a plot of minimum detectable source amount versus cargo depth is produced for each algorithm. These results are discussed further in Section 5. 


\section{Commodity Study}

As part of this project, we analyzed US import data collected during all of 2006 on containers shipped through US ports - over 4.5 million containers. Using these data, we extracted a variety of distributions of interest to modelers and developers of active and passive detection systems used to scan IMCCs for potential contraband. This work expands on some of the analysis presented in an earlier report from LLNL by investigating the foreign port distribution of commodities shipped to the US [12]. Details of this present analysis are given in a separate report [24].

The data used in this report were obtained from Port Import Export Reporting Service (PIERS) Global Intelligence Solutions, hereinafter referred to as PIERS. ${ }^{1}$ The company collects data from more than 15 million bills of lading per year, which translates to more than 20 million shipments, with data as far back as 1950. PIERS processes these data into databases, facts, and figures, which others can then use to better understand the global trade market. With the database we obtained, we were able to better understand the dynamics of what commodities are shipped to the US, from which ports, and in what quantities. Each commodity has a specific Harmonized System (HS) Code-a numerical identifier for the commodity.

The majority of containers shipped to the United States are 40 - $\mathrm{ft}$ containers $(\sim 70 \%)$; about $25 \%$ are $20-\mathrm{ft}$; and about $3.6 \%$ are 45 -ft containers. A small fraction $(<1 \%)$ of containers are of other, more specialized sizes, and very few ports actually ship these unique size containers. The primary foreign ports that ship the largest numbers of each container size are shown in Table 2 below. Given that 45-ft containers comprise 1 of out every 27 containers shipped to the US, and considering the foreign ports from which they are shipped, they should not be ignored in screening; further testing and analysis of radiation measurements for national security with this size container is warranted.

Table 2. Summary of Top 10 International Ports for the Three Major Container Sizes Shipped to the US

\begin{tabular}{|c|c|c|c|c|c|c|c|c|c|}
\hline \multirow[b]{2}{*}{$\begin{array}{c}\text { Ran } \\
\mathbf{k}\end{array}$} & \multirow[b]{2}{*}{ Port } & \multicolumn{2}{|c|}{ 20-ft Containers } & \multirow[b]{2}{*}{ Port } & \multicolumn{2}{|c|}{ 40-ft Containers } & \multirow[b]{2}{*}{ Port } & \multicolumn{2}{|c|}{ 45-ft Containers } \\
\hline & & Number & $\begin{array}{l}\% \text { of } \\
\text { Total }\end{array}$ & & Number & $\begin{array}{l}\% \text { of } \\
\text { Total }\end{array}$ & & $\underset{r}{\text { Numbe }}$ & $\begin{array}{l}\% \text { of } \\
\text { Total }\end{array}$ \\
\hline 1 & Shanghai & 159,507 & 8.60 & Yantian & 778,866 & 15.25 & Yantian & 75,824 & 28.94 \\
\hline 2 & Busan & 143,284 & 7.72 & Shanghai & 594,462 & 11.64 & Hong Kong & 33,541 & 12.80 \\
\hline 3 & Kaoshiung & 128,149 & 6.91 & Hong Kong & 417,513 & 8.17 & Shanghai & 26,933 & 10.28 \\
\hline 4 & Hong Kong & 94,618 & 5.10 & Busan & 348,586 & 6.82 & PTO Cortes & 17,545 & 6.70 \\
\hline 5 & Singapore & 71,095 & 3.83 & Kaoshiung & 317,897 & 6.22 & San Juan & 16,443 & 6.28 \\
\hline 6 & Yantian & 69,585 & 3.75 & Ningpo & 164,551 & 3.22 & Kaoshiung & 16,344 & 6.24 \\
\hline 7 & Freeport & 55,450 & 2.99 & Bremerhaven & 154,928 & 3.03 & Singapore & 9,018 & 3.48 \\
\hline 8 & Antwerp & 52,231 & 2.82 & Singapore & 146,296 & 2.86 & Rotterdam & 6,549 & 2.50 \\
\hline 9 & Santos & 48,775 & 2.63 & Rotterdam & 123,913 & 2.43 & Xiamen & 6,115 & 2.33 \\
\hline 10 & Qingdao & 47,773 & 2.58 & Qingdao & 103,273 & 2.02 & STO Tomas & 5,613 & 2.14 \\
\hline
\end{tabular}

\footnotetext{
${ }^{1}$ Piers Global Intelligence Solutions, 2 Penn Plaza East, $12^{\text {th }}$ Floor, Newark, New Jersey, USA.
} 
Analysis of all containers can provide a wealth of information. Access to the database makes it possible to determine which general commodities are shipped overseas in IMCCs more frequently. Commodities are generally shipped with a 4- or 6-digit HS code that describes the commodity. These codes can be grouped by a collapsed 2-digit HS Code that represents the commodities. This shipping data is generalized into approximately 16 categories that span the range of commodities. This was performed by collapsing the two-digit codes into general categories specified by Foreign Trade OnLine Corporation. These data are shown in Table 3 below. It should be noted that HS codes 90-97 are generally referred to as a 'miscellaneous' category, but we did not collapse the HS codes in this major category because these commodities represent a large fraction of the total number of containers shipped to the US (particularly 40- and 45-ft containers), and the commodities grouped therein are quite different from one another. The breakdown for all 97 HS codes is shown in Appendix B of the separate, detailed report [24].

Table 3. Shipping Container Data Arranged by Major HS Code Categories

\begin{tabular}{|c|c|c|c|c|c|c|c|}
\hline $\begin{array}{c}\text { HS } \\
\text { Code } \\
\text { Rang } \\
\mathrm{e}\end{array}$ & Description & $\begin{array}{c}20-\mathrm{ft} \\
\text { Containers }\end{array}$ & Percent & $\begin{array}{c}40-\mathrm{ft} \\
\text { Containers }\end{array}$ & Percent & $\begin{array}{c}45-\mathrm{ft} \\
\text { Containers }\end{array}$ & Percent \\
\hline 00 & Household Goods & 49,895 & 2.69 & 199,852 & 3.91 & 12,032 & 4.59 \\
\hline 01-05 & $\begin{array}{l}\text { Animal and Animal } \\
\text { Products }\end{array}$ & 45,524 & 2.45 & 104,827 & 2.05 & 268 & 0.10 \\
\hline $06-15$ & Vegetable Products & 128,119 & 6.91 & 227,331 & 4.45 & 964 & 0.37 \\
\hline $16-24$ & Foodstuffs & 182,724 & 9.85 & 280,804 & 5.50 & 3750 & 1.43 \\
\hline $25-27$ & Mineral Products & 74,358 & 4.01 & 16,114 & 0.32 & 138 & 0.05 \\
\hline $28-38$ & $\begin{array}{l}\text { Chemical \& Allied } \\
\text { Industries }\end{array}$ & 163,034 & 8.79 & 128,406 & 2.51 & 5162 & 1.97 \\
\hline $39-40$ & Plastics \& Rubbers & 128,262 & 6.91 & 455,844 & 8.92 & 13,948 & 5.32 \\
\hline $41-43$ & $\begin{array}{l}\text { Raw Hides, Skins, Leather } \\
\text { \& Furs }\end{array}$ & 11,085 & 0.60 & 83,440 & 1.63 & 7767 & 2.96 \\
\hline $44-49$ & Wood \& Wood Products & 120,774 & 6.51 & 391,535 & 7.66 & 10,176 & 3.88 \\
\hline $50-63$ & Textiles \& Clothing & 67,524 & 3.64 & 382,513 & 7.49 & 51,426 & 19.63 \\
\hline $64-67$ & Footwear \& Headgear & 15,108 & 0.81 & 108,316 & 2.12 & 18,999 & 7.25 \\
\hline $68-71$ & Stone \& Glass & 238,465 & 12.85 & 156,979 & 3.07 & 5203 & 1.99 \\
\hline $72-83$ & Metals & 299,302 & 16.13 & 316,307 & 6.19 & 18,730 & 7.15 \\
\hline $84-85$ & Machinery \& Electrical & 174,963 & 9.43 & 801,411 & 15.69 & 22,395 & 8.55 \\
\hline $86-89$ & Transportation Products & 80,965 & 4.36 & 293,234 & 5.74 & 12,384 & 4.73 \\
\hline 90 & $\begin{array}{l}\text { Photograph and Medical } \\
\text { Instruments }\end{array}$ & 10,437 & 0.56 & 50,093 & 0.98 & 4898 & 1.87 \\
\hline 91 & Clocks \& Watches & 684 & 0.04 & 4905 & 0.10 & 566 & 0.22 \\
\hline
\end{tabular}




\begin{tabular}{|c|c|c|c|c|c|c|c|}
\hline $\begin{array}{c}\text { HS } \\
\text { Code } \\
\text { Rang } \\
\text { e }\end{array}$ & Description & $\begin{array}{c}20-\mathrm{ft} \\
\text { Containers }\end{array}$ & Percent & $\begin{array}{c}40-\mathrm{ft} \\
\text { Containers }\end{array}$ & Percent & $\begin{array}{c}45-\mathrm{ft} \\
\text { Containers }\end{array}$ & Percent \\
\hline 92 & Musical Instruments & 1566 & 0.08 & 8786 & 0.17 & 478 & 0.18 \\
\hline 93 & Arms \& Ammunition & 1068 & 0.06 & 1109 & 0.02 & 8 & 0.00 \\
\hline 94 & Furniture & 31,576 & 1.70 & 816,169 & 15.98 & 38,074 & 14.53 \\
\hline 95 & $\begin{array}{l}\text { Toys, Games \& Sports } \\
\text { Equipment }\end{array}$ & 25,360 & 1.37 & 255,781 & 5.01 & 33,013 & 12.60 \\
\hline 96 & Manufactured Articles & 3682 & 0.20 & 20,819 & 0.41 & 1325 & 0.51 \\
\hline 97 & Works of Art \& Antiques & 766 & 0.04 & 4113 & 0.08 & 268 & 0.10 \\
\hline
\end{tabular}

While a large amount of NORM is shipped in IMCCs, only a few specific commodities are shipped with enough frequency to present potential issues in screening IMCCs at ports that handle overseas shipping containers. The majority of containers with NORM will contain fertilizers $(5,700$ containers), granite $(59,000$ containers), or ceramic $(225,000$ containers) materials. Fertilizers were generally shipped in either 20 - or 40 -ft containers with equal frequency. While granite is mostly shipped in 20-ft containers, ceramic materials can be shipped in either 20- or 40-ft containers. The size of container depended on the specific use of the ceramic or porcelain material. General construction ceramics (such as floor and roofing tiles) tend to be shipped in 20-ft containers. Consumer products made from ceramic materials (e.g., tableware, sinks, and toilets) are generally shipped in 40-ft containers. This discrepancy is due in large part to the packaging of the commodity. Consumer products are generally shipped packed in a box loaded with Styrofoam ${ }^{\mathrm{TM}}$ or other packing material to protect the product from breakage. Construction ceramic materials are generally shipped in less packing material, many times consisting of only a cardboard or wooden box. Granite is almost always shipped in a 20 -ft container, due to its very high density. Thus, signatures from certain commodities containing NORM can be associated with specific container sizes.

A previous report [12] contained analysis of weight distributions based on twenty-foot equivalent units (TEU), which normalized all container sizes. However, it is probably more illustrious to investigate these distributions based on container size. This is demonstrated in Figure 30, which shows distinct distributions for the three different containers. For $20-\mathrm{ft}$ containers, the distribution is relatively flat below 30,000 lbs., but has two peaks centered around 37,000 and 43,000 lbs., respectively. The 40 -ft container distribution has a large, broad peak below 30,000 lbs., with a maximum around 14,000 lb. There is also a peak around 42,000 lbs., and a relatively flat distribution from 50,000-57,000 lb. Forty-five-foot containers had a very broad distribution from 5000$45,000 \mathrm{lbs}$. /container, with a maximum near 17,500 lb. The distribution for the dominant, $40-\mathrm{ft}$ containers is used below in our analysis of the performance of an SBRD system. 
PNNL-21948

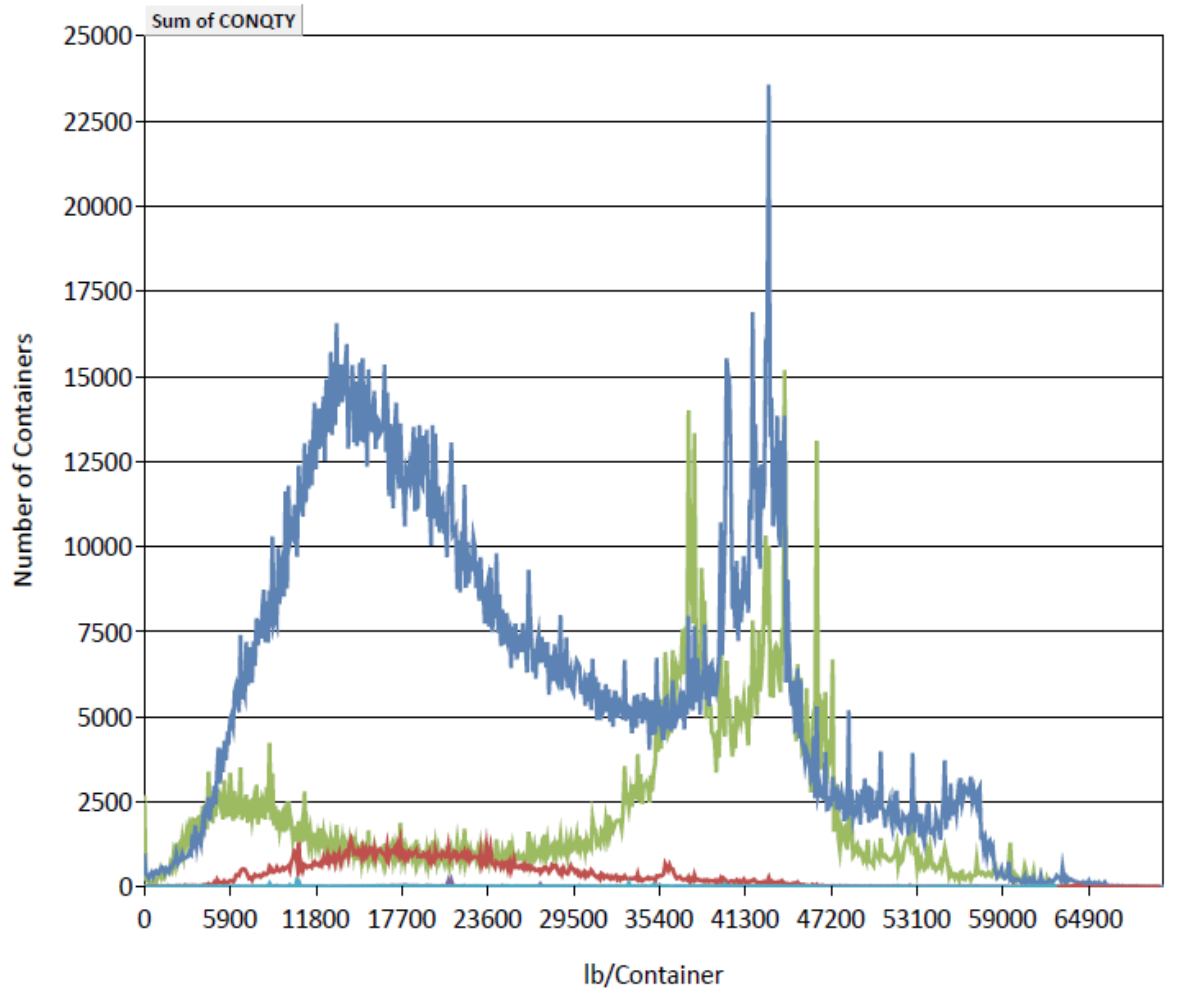

Figure 30. Weight distribution of all containerized commodities entering the United States for 45 -foot containers (red curve) 40-foot containers (blue curve) and 20-foot containers (green curve). 
PNNL-21948

\section{$5 \quad$ Results and Discussion}

\subsubsection{Algorithm Results}

Results from the "New SBRD" detector have been analyzed in the style of the last report from March 2010, in which the "SBRD" and "Enhanced SBRD" were compared [10-11]. MCNP calculations of a $1-\mathrm{kg}$ HEU source at various heights in a full load of wood in a 40-foot IMCC were used to calculate the MDQ as a function of depth for various algorithms (Figure 31).
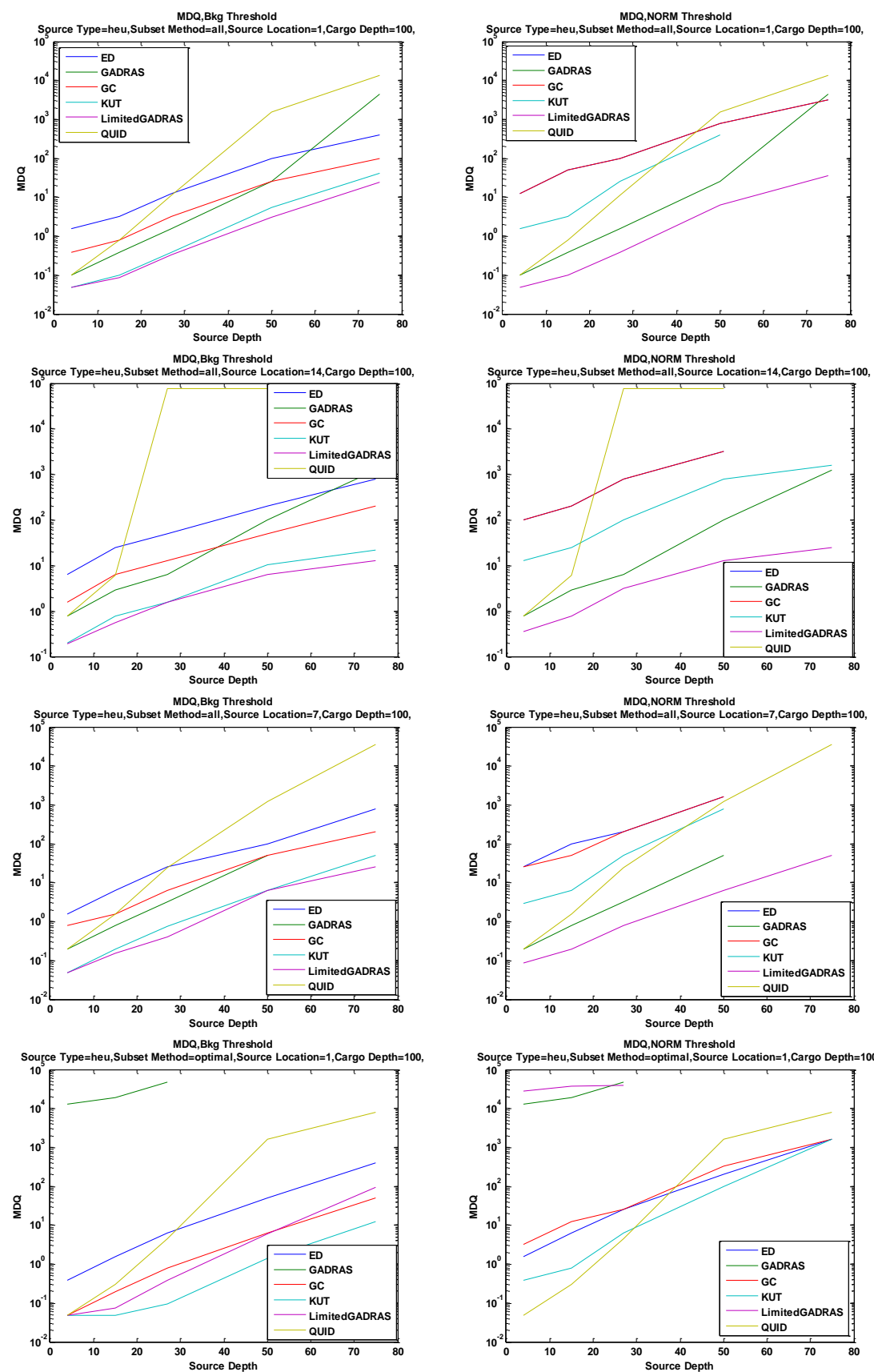
PNNL-21948
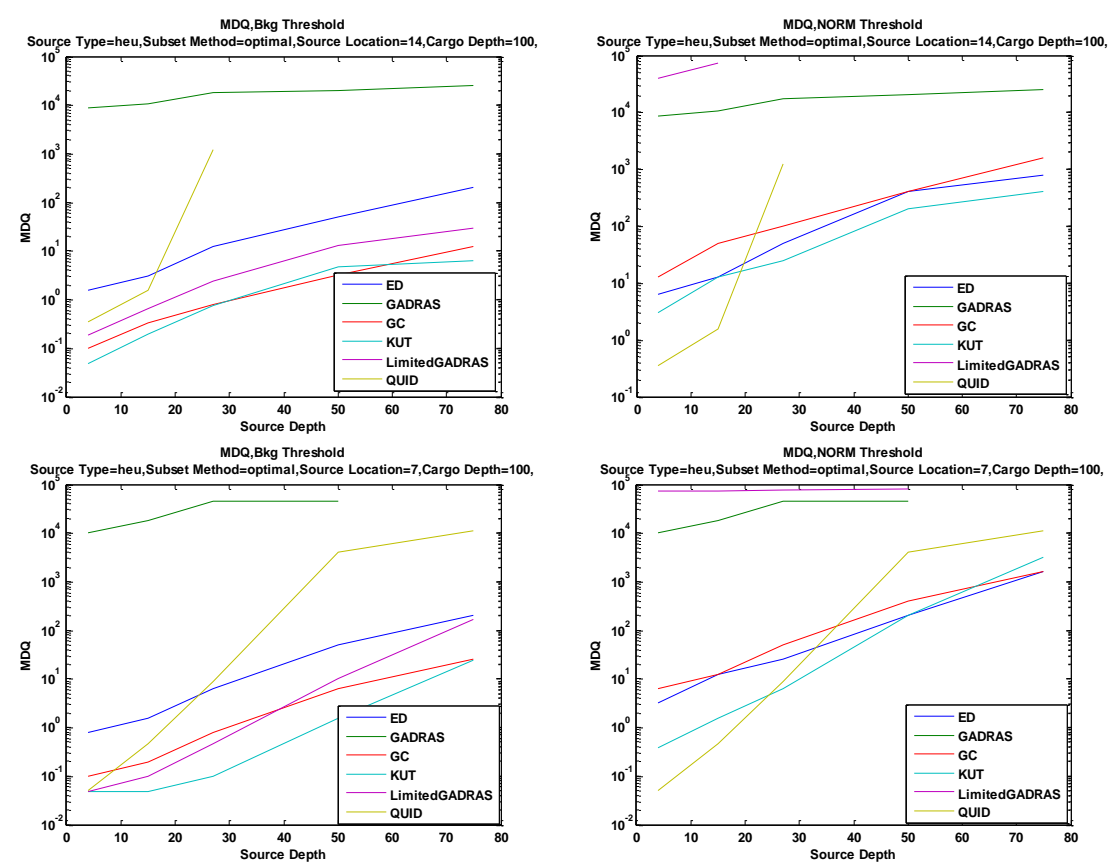

Figure 31. MDQ for various algorithms, with and without optimal subset method.

Several results are of special note here. As would be expected, algorithms that rely on specifics of the spectra such as peak shape or templates tend to degrade more quickly as shielding becomes large. However, these sorts of algorithms perform well with light shielding and showed reasonable capability to identify sources at these levels. However, the inclusion of NORM degraded the performance of many algorithms somewhat, particularly in the case of the gross count (GC) and energy distance (ED) metrics, the results of which are coincident in many cases. In general, when the ED line is not visible it is equivalent to the GC result. Algorithms that account for background fluctuations such as the QuID and GADRAS algorithms seem to be affected the least by the requirement to exclude NORM, allowing for substantially better detection when NORM is present.

The GADRAS and Limited GADRAS results deserve some specific consideration. The addition of NORM substantially impacts the specific algorithms, with the exception of GADRAS. The GADRAS algorithm was run under the assumption that no nuisance alarms due to NORM were present. The inclusion of these effects it makes GADRAS almost entirely untenable when NORM is a consideration, as the spurious detection of additional isotopes not actually present leads to a substantial false alarm rate. For this reason, the GADRAS result is not perfectly analogous to the others, as the requirement on false alarm rate makes its use nearly impossible in a real way. However, the Limited GADRAS approach (in which a limited set of benign spectra are used) removes this issue and allows the inherent power of the template matching scheme to be used without relying on an expanded catalog of sources.

It's also worth noting that these results vary strongly with and without the application of "optimal" subsets. Using the optimal subset method dramatically improves the performance for most algorithms but actually seems to hurt the performance of GADRAS and Limited GADRAS, due to the fact that additional noise due to fewer detectors leads to the detection of incorrect isotopes. In the case of Limited GADRAS, the use of only a few detectors for the "optimal" set leads to a very erratic result, and a few NORM results therefore have high chi square values. The regular GADRAS implementation simply does not evaluate the counts from the limited detector set as sufficient to estimate HEU, and as a result does not return this result. Thus, while GADRAS may not be the best 
choice for this kind of detection due to the high nuisance incidences, Limited GADRAS may still be among the optimal choices when all the available detectors are used to limit false identifications.

The QuID algorithm maintains the best result for the relatively unshielded source, providing the best possible result at low depths but quickly degraded as more limited spectral information is available due to attenuation and down scattering by cargo.

For the best results estimated in this way, the KUT algorithm used with a limited subset of detectors, or the Limited GADRAS approach with the entire detector set may be optimal. In these cases, MDQ rises from a very low level of a fraction of a source in the unshielded case, to between 1 and 10 sources worth of flux over the first $50 \%$ of cargo depth. This is in keeping with the results from the previous study [10-11] with realistic background attenuation but more detector material. Even after NORM is added as a consideration, the best-performing algorithms may be able to detect the presence of the stated sources near the center of the cargo container at the level of a few test sources worth of flux. This relatively optimistic result is found by exploiting the capability of anomaly algorithms to provide insensitivity to NORM while detecting illicit sources. As both of the best results found by this work use similar methods for detection (Limited GADRAS and the KUT approach both operate by using a limited set of spectra for fitting, and employ a goodness-of-fit metric as an anomaly detector), these approaches are expected to be the best for the operation of a fielded detection system. It is possible that multiple algorithms may be used in parallel, for example the pairing of an algorithm which uses peak finding (for use when peaks are easily discernible) with an anomaly detector when peaks are not obviously present in the data. It is expected that this sort of hybrid approach may combine the best performance at both low and high attenuation.

\subsubsection{Commodity Distribution}

The distribution of 40-foot cargo containers entering the USA as a function of weight of the cargo was also determined for comparison with the detector performance to varying overall cargo amount. The weight distribution of all containers coming into the United States was described previously and shown in Figure 30. The 40-foot container distribution was normalized and the fraction of containers (intermodal cargo container or IMCC) versus cargo weight is shown in Figure 32.

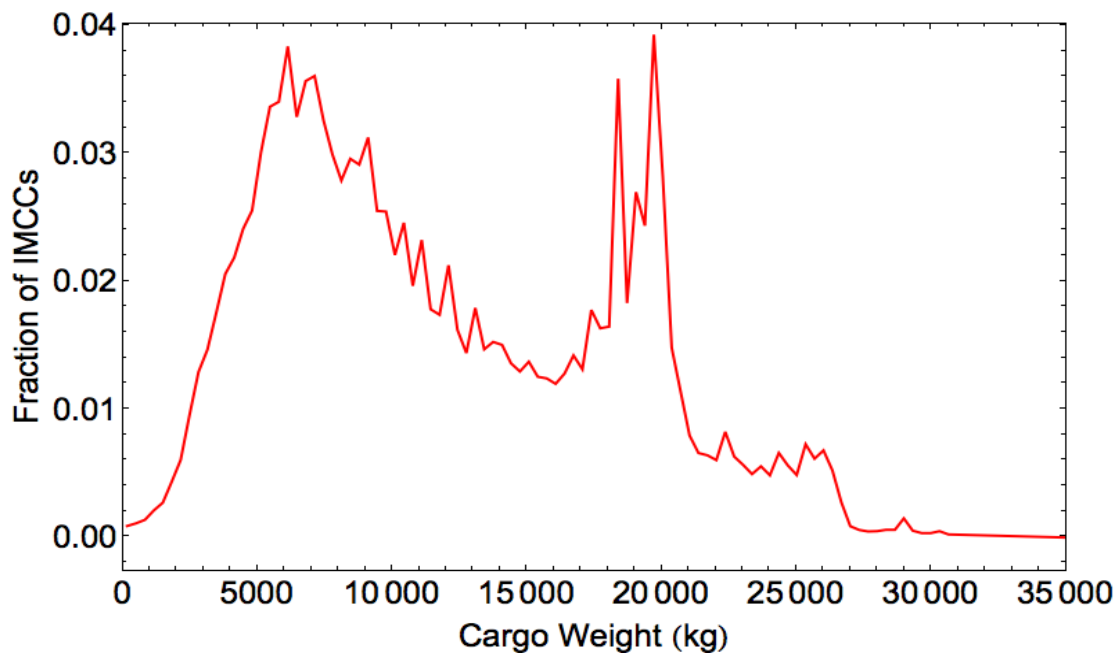

Figure 32. Forty-foot container prevalence by weight. 
This distribution was in turn used to generate the fraction of 40 -foot containers versus wood cargo depth assuming a constant cargo density equal to the wood cargo model used for the simulations in this work, $0.46 \mathrm{~g} / \mathrm{cm}^{3}$ (Figure 33). A substantial fraction of cargo containers contain less than around 1.1 meter of equivalent wood shielding in terms of overall mass, corresponding to a cargo container around $50 \%$ full of wood.

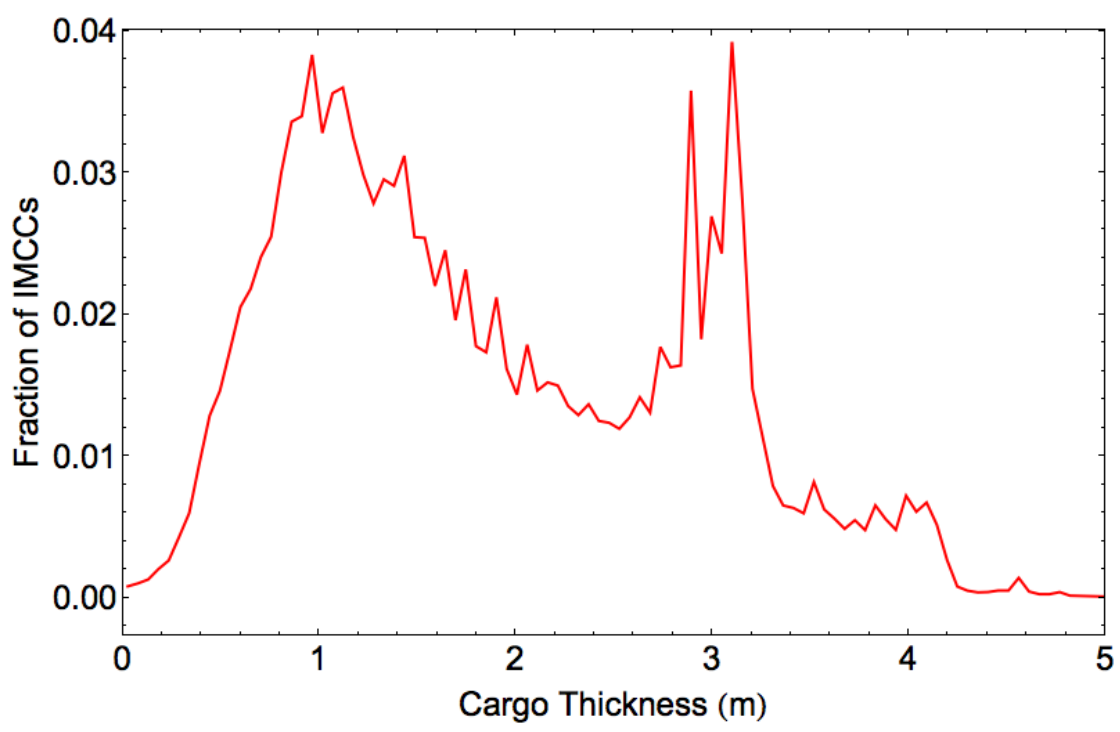

Figure 33. Forty-foot container prevalence by equivalent cargo thickness.

\subsubsection{Enhancements}

To create a performance estimate for the enhanced SBRD system (New SBRD), the corresponding performance for the SBRD system was evaluated at the detection depth corresponding to a MDQ of $10 \mathrm{~kg}$ of HEU. This is shown in Figure 34, where due to the limited simulations accomplished in this study, the distribution curve of the previous study was incorporated [10-11]. This performance was then scaled by the MDQ estimates produced by this work to give the estimates shown in Figure 35 and Figure 36. In each plot the upper curve for the New SBRD corresponds to the easiest location for detection (in the middle of the container), while the lower curve corresponds to the most difficult source position, which is in the corners of the container.

These results for the enhanced SBRD are similar to the previous study (as shown in the introduction and described in Appendix B), but provide a much higher level of robustness, as these estimates include validation of the model to measurements, and using detection algorithms instead of an estimation of detection capability. 
PNNL-21948

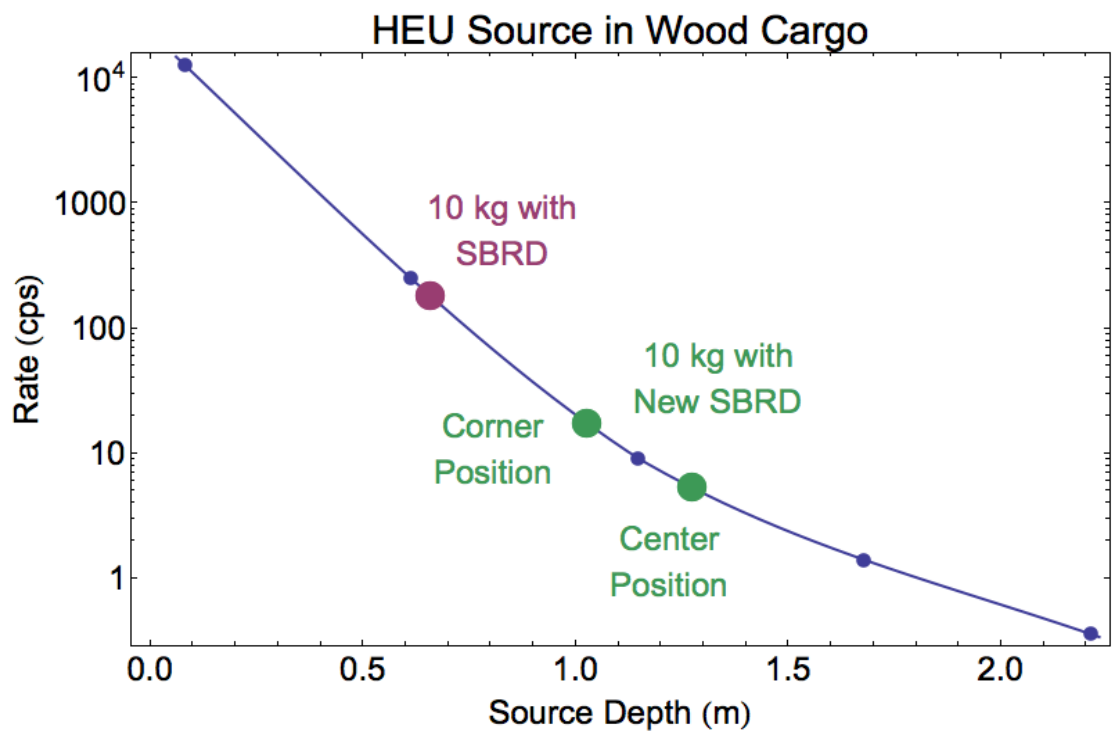

Figure 34. Simulated count rate as a function of source depth in the cargo for HEU.

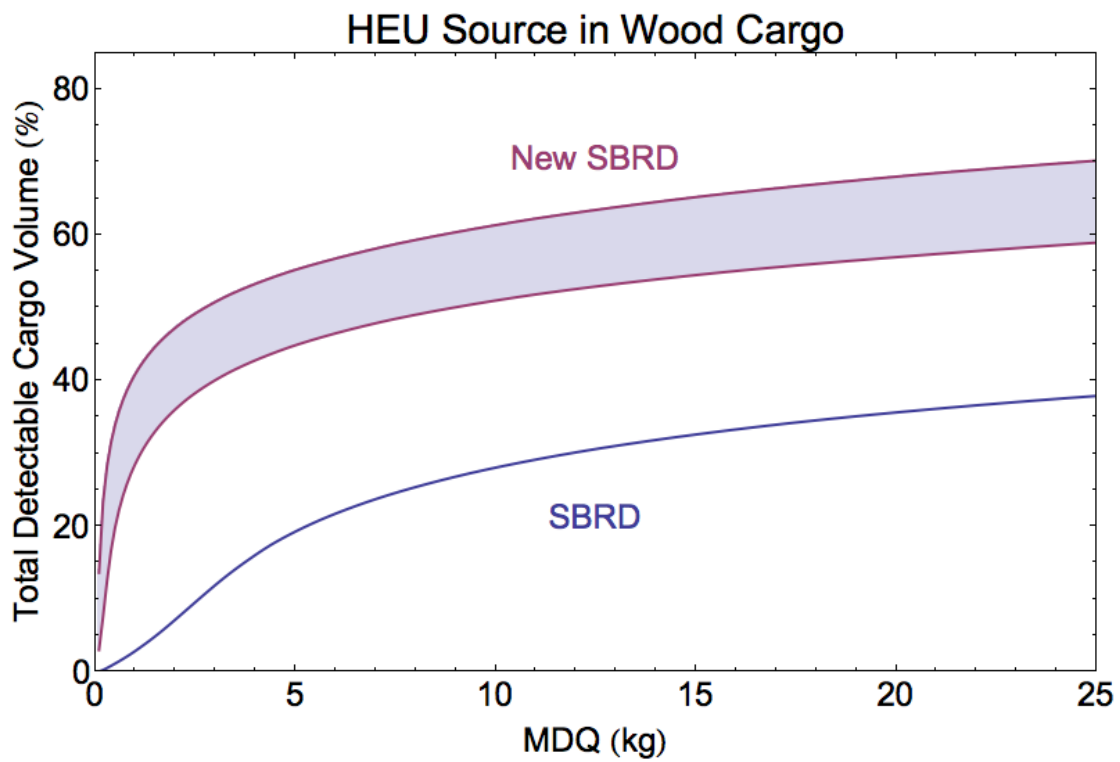

Figure 35. Maximum cargo thickness for HEU source detection. 
PNNL-21948

HEU Source in Wood Cargo - Worst Case

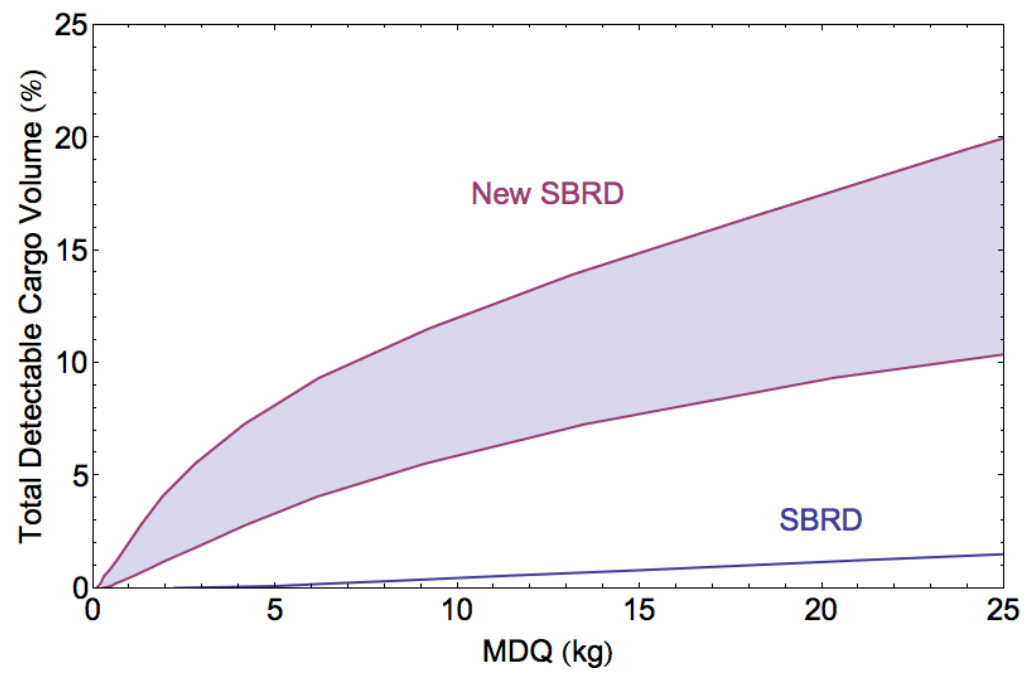

Figure 36. Maximum Cargo thickness for HEU Source Detection, Worst Case. 
PNNL-21948

\section{Conclusions and Path Forward}

\subsection{Conclusions}

The combination of the best available detection algorithms and the enhanced SBRD model provides a significant estimated increase to overall sensitivity of the spreader bar detector system as compared to the commercial systems as tested in 2008, by an approximate increase of a factor of two for HEU. While this enhancement is most prominent in cases of light shielding (i.e., a limited overall cargo depth), the combination of additional detector elements, optimal detector combination and anomaly detection algorithms provides an enhancement across shielding depths. However, the one-sided nature of the SBRD presents intrinsic limitations to overall sensitivity, leading to high MDQs for many detection scenarios. In particular, while the enhanced SBRD may achieve an MDQ of around $10 \mathrm{~kg}$ for a substantial fraction of cargo containers entering the US, this figure is not the case for the most challenging source locations, where the MDQ may be unreasonably large.

Significant work was performed on both the algorithm development and the container cargo distributions entering the US, and the result of this modeling, validation, and simulation effort was a set of estimates of the performance of a variety of radiation detection algorithms in this cargoscreening environment.

In the case of algorithms, a variety of contemporary radiation detection and identification approaches were employed, and the optimal approaches to selecting time and location subsets of detector data were explored. In all cases, algorithm performance was compared, and the best approaches were suggested along with the best expected performance from the enhanced SBRD. In general, templatematching schemes employing a limited set of regression parameters tend to provide best results overall including the deep shielded scenarios. For a central source location, these improvements may allow the enhanced SBRD to achieve an effective Minimum Detectable Quantity (MDQ) around 10 $\mathrm{kg}$ of HEU positioned within around a 50\% full cargo container, assuming nominal cargo density. However, the exponential attenuation due to additional dense cargo makes detection difficult behind any larger amounts of cargo.

The improvements of an enhanced SBRD with increased detector material and improved algorithms result in an effective MDQ of $10 \mathrm{~kg}$ of HEU in 50 percent of the imported cargo in 40-foot containers, assuming a random source location. This is approximately two times the capability of the estimated performance of the commercial systems (based on 2008 configurations). For an MDQ of the $10 \mathrm{~kg} \mathrm{HEU}$ source located in the worst location (bottom of the 40-foot container), the volume of cargo scanned drops to $10 \%$ for the enhanced SBRD, again a factor of two or more larger than the commercial systems. Enhancing the SBRD systems by adding more detectors, providing a more uniform coverage, and incorporating more advanced algorithms will result in a significant increase in capability.

\subsection{Path Forward}

This study has provided some indications of the possible improvements and limitations of a spreaderbar radiation detection system. This work was focused on HEU detection in a 40-foot container, additional investigation for other threat material, as well as other container configuration will help 
map out the possibilities for SBRD systems. The results here can be used along with other studies to support the development of specific requirements for a system of this type in the future.

The additional capability of the enhanced SBRD model, combined with the difficulty of detection through high levels of intervening cargo suggest that the single sided nature of the SBRD will limit the potential for such a screening system. The system could be further enhanced with a basic variation to detector footprint and shape (e.g., a detector with additional side panels) or a combination of detection approaches (e.g., the combination of a spreader-bar detection system with a detector panel beneath the roadway to accomplish two-sided detection).

The additional sensitivity of advanced algorithmic approaches is also of interest to the advancement of multiple-detector-element architecture of this sort, and combined or further algorithmic advancements may allow for further increases to detection sensitivity.

Although this work included some validation with the SNL developed system, additional verification with additional experimental measurements and simulations would provide increased confidence in the models and results provided here, and lead to extending the simulations beyond the limited scope of this study. 
PNNL-21948

\section{References}

1. "Detecting Illicit Nuclear Materials." RT Kouzes, 2005, American Scientist, (September-October 2005) 93:422-427.

2. "Monitoring of Seaport Container Cargo with Gantry Crane Mounted Radiation Detection Equipment," J Evans, 2004, PIET-43741-TM-071, PNNL-14732

3. "Suitability of Seaport Spreader Bar Radiation Detection Systems," SM Robinson and JE Schweppe, PIET-43741-TM-475, PNNL-17142

4. "Intermodal Cargo Container Screening - Single Side Screening Technical Analysis," SM Robinson, JE Schweppe, RJ McConn Jr, RT Pagh, and ER Siciliano, 2007, PIET-43741-TM-523, PNNL-16879

5. "Scanning Cargo with a Crane-Mounted Detector at Seaports," RJ McConn Jr, SM Robinson, and JE Schweppe, preliminary draft, PIET-43741-TM-733

6. "Operational Testing of Crane Mounted Radiation Detection Systems", Report to Congress, 2008, Submitted by CBP

7. "Phase II Test Report for Spreader Bar Radiation Detectors," JH Ely, KR McCormick, DC Stromswold, and PS Stansbury, 2008, PIET-43741-TM-797, PNNL-17971

8. "SBRD Test Report," S Croney, D Miko, D Desimone, A Niemeyer, and J Toevs, 2009, Los Alamos National Laboratory report LA-CP-09-00436

9. "Benchmark Sources for Radiation Detection Architecture Evaluation (revision 2)," P Sokkappa, C Guenther, B Pohl, S Sitaraman, E Smith, and R Hillaire, 2007, LLNL report UCRL-TR218277-REV-2, Lawrence Livermore National Laboratory, Livermore, CA.

10. "Performance Assessment of Spreader-Bar Radiation Detection Systems for the Detection of Special Nuclear Material in Cargo", JH Ely, LE Erikson, NR Hilton, GA Kallenbach, GA Sandness, SM Robinson, and JE Schweppe, 2009, SAND2009-7998P, PNNL-19036

11. "Status of the Spreader-Bar Radiation Detector (SBRD) Investigations", JH Ely, LE Erikson, NR Hilton, GA Kallenbach, GA Sandness, SM Robinson, and JE Schweppe, 2010, SAND20101376P, PNNL-SA-71406

12. "Analysis of Recent Manifests for Goods Imported through US Ports", MA Descalle, D Manatt, and D Slaughter, 2006, Lawrence Livermore National Laboratory report UCRL-TR-225708

13. X-5 Monte Carlo Team, MCNP-A General Monte Carlo N-Particle Transport Code, Version 5 Vol. I: Overview and Theory, 2003, revised 2008, Los Alamos National Laboratory Report LAUR-03-1987

14. "Investigation of Source Detection Algorithms for Gross Counting Portal Monitors," CG Wahl, D Alderson, L Pibida, 2007. IEEE Sens. Appl. Symp. SAS

15. "Examination of Count-Starved Gamma Spectra using the Method of Spectral Comparison Ratios," RC Runkle, DM Pfund, KK Anderson, KD Jarman, 2006. IEEE Nucl. Sci. Symp. Conf. Record, 1-6 (2006), pp. 70-76

16. "Evaluation of Key Detector Parameters for Isotope Identification," CJ Sullivan, SE Garner, M Lombardi, KB Butterfield, MA Smith-Nelson, 2007. IEEE Nucl. Sci. Symp. Conf. Record (2007), p. 4.

17. "Rapid Computation of Gamma-ray Spectra for One-Dimensional Source Models", DJ Mitchell and J Mattingly, 2008, Trans. Am. Nucl. Soc. 98, 565, (2008).

18. "An isotope identification injection study with GammaTracker," MT Batdorf, LE Erikson, CE Seifert, MJ Myjak, LJ Kirihara, Oct. 2011, Nucl. Inst. Meth. A, Volume 652, Issue 1, Pages 116119.

19. "MCNP "thin Disk" Background model." ER Siciliano, and SM Robinson, 2007, PNNL-SA58383, Pacific Northwest National Laboratory, Richland, WA. 
20. "Low Count Anomaly Detection at Large Standoff Distances," DM Pfund, KD Jarman, BD Milbrath, SD Kiff, and DE Sidor, 2010, IEEE Transactions on Nuclear Science 57(1, PT 2):309316.

21. "Statistical methods applied to gamma-ray spectroscopy algorithms in nuclear security missions," DK Fagan, SM Robinson, RC Runkle, 2012, Applied Radiation and Isotopes, Volume 70, Issue 10, October 2012, Pages 2428-2439.

22. "Deconvolution and Background Subtraction by Least-Squares Fitting with Prefiltering of Spectra", PJ Statham, 1977, Analytical Chemistry, Vol. 49. No 14.

23. "Evaluation of Three Zero-Area Digital Filters for Peak Recognition and Interference Detection in Automated Spectral Data Analysis", F Janssens and J Francois, 1991, Analytical Chemistry, Vol. 63, No. 4, Feb 1991.

24. "Analysis of International Commodity Shipping Data and the Shipment of NORM to the United States,” JE Baciak, GA Sandness, JH Ely, SM Robinson, and JE Schweppe, 2011, PNNL-21088, Pacific Northwest National Laboratory, Richland, WA. 


\section{Appendix A: File Generation and Structure}

These files are created by a MATLAB ${ }^{2}$ script which samples each data file according to each optimal subset for a source position and depth, creating single-spectrum, summed data files with file names in the format:

Data _ <subset method> _ <source, Bkg, or NORM> _ 1sec _ <depth>depth _ newdetector _ pos_ <source position used for subset> _ depth _ <source depth used for subset>

Here, the first "depth" number has multiple meanings, referring to the source depth within full cargo for sources, and the depth of cargo for background (and NORM).

\section{$\underline{\text { ASC scenario files }}$}

The current software used to produce ASC scenario files from raw data and MCNP inputs (ASC_stochastic_generator_v15_forSpreaderBar.rb) is written in the Ruby language, and provides statistical realizations of scenarios to be analyzed by detection/ID algorithms. To provide this code with useful data, MATLAB code is written to input the source information and the various subsets, and appropriate combinations of the source, background and NORM data will be written to flat spectra files that correspond to the summed inputs for 30 seconds.

The data output from the MATLAB stage is given to the ASC software such that optimal subsets can be constructed for each source location beforehand, and then used to generate the ASC "scenario files" used to estimate algorithm performance. In this stage all of the data variations are taken in, and at present, the standard HEU source, background, and NORM source expected to represent a relatively "heavy" NORM source.

All subsets, necessary to the operation of the algorithms, will be produced, each with the optimal, total, or random detector set. Then the .ASC writer stage will be used to generate the following scenario data, each realized for 30 seconds and for 50 different realizations, each with the appropriate background:

All sources (including "no source")

All locations/depths

All (appropriate) cargo depths

Each NORM type

Because of the additional procedural complexity associated with the preceding stages, efforts will be made to take these files and spot-check them for accuracy and find any potential errors. From each subset of data, a few foreground, background and net spectra will be inspected and verified to be as expected from the original MCNP outputs.

Here are some spectra associated with the "optimal subset" data. ASC files are kept in the format:

<source>_<subset>_<sourceLocation>_<sourceDepth>_<cargoDepth>_<sourceStrength>_<iterationNumber $\rangle_{\text {.ASC }}$

This format will be used to hold source, background ( $\mathrm{src}=0)$, and NORM simulated data.

Source='BKG' runs use the empty cargo container for both attenuated and un-attenuated background. These scenarios are meant to simulate a truly empty cargo container. HEU sources with 0 source

\footnotetext{
${ }^{2}$ Available from The MathWorks, Inc. at http://www.mathworks.com/products/matlab/.
} 
strength, on the other hand, represent scenarios with a container full of cargo but no source. To ensure an acceptably low false alarm rate, both sorts of scenarios must be excluded by setting sufficiently high algorithm thresholds.

In order to check these files, several inspections are performed. First, a collection of spectra from each ASC file is displayed for consistency. These spectra should reproduce the HEU energy structures at the appropriate locations and show that background and foreground are similar when source is small. In fact, as the attenuated background uses a full cargo container while the previously measured background has an empty one, it should be somewhat smaller. These things are verified visually first.

From these results, we can see the following specific effects, which we expect:

- Everything which begins with the same beginning (e.g. heu_optimal_1_0_100_xxx_y) should have the same background, as the same subsets and data are used

- The backgrounds which are made using different subsets should scale with the number of crystals in that subset (more or less, since not all crystals seem the same background)

- The foreground data in a no-source run should be different from the background, and the background should actually be larger, as it is considering an empty cargo container while the "foreground" uses a full one.

Thereafter, a few quantitative measurements are made to verify the quantitative operation of the code. Specifically, for the optimal subset summation, and considering a strong HEU source at around 50\% cargo depth, the gross-count sum in the ASC file is compared with the original data (as gotten from the original .xls files) for consistency. Here we choose the ASC file "heu_optimal_1_4_100_819.2_33.ASC." The foreground (summed over all the bins) for this file is $1.13 \mathrm{E} 8$, while the background is 5.6E3. Now, the detectors used to establish the "optimal subset" for these data (location 1, depth 4\%) contains detectors number 9, 12, and 13. Looking at the files HEU_1sec_4depth_32detector.csv, BKG_1sec_100depth_32detector.csv and BKG_1sec_4depth_32detector.csv, we find the following sums from adding all the non-zero bins in detectors 9,12 , and 13 from position 1:

HEU_1sec_4depth_newdetector.csv: 4.77E3

BKG_1sec_100depth_newdetector.csv: 30.8

BKG_1sec_0depth_newdetector.csv: 187.2

Now, we expect the background from the corresponding ASC file to be drawn from the background of an empty container, over 30 seconds, or sum to around $187.2 * 30=5.6 \mathrm{E} 3$, which is accurate. Likewise, we expect the foreground to be the sum of the remaining two (attenuated background and source times 819.2), or:

$(30.8+(4.77 \mathrm{E} 3 * 819.2)) * 30=1.17 \mathrm{E} 8$, which is also approximately correct.

Given the result from this spot-check, we believe that the summation rules are being handled correctly all the way through the production of .ASC scenario files.

\section{Algorithm Results}

Now, each algorithm is run over every .ASC file. To recap, the ASC files we read in will all have filenames like this:

heu_optimal_1_0_0.05_1.ASC 
These files have the format:

<source>_<subset>_<sourceLocation>_<sourceDepth>_<cargoDepth>_<sourceStrength>_<iterationNumber $\rangle_{\text {.ASC }}$

These are laid out as follows:

Source: These currently include "heu," "norm" and "bkg". More sources may be added.

Subset: "optimal" means the best subset found by the method described earlier, "all" means every crystal is summed together, and "random" means that the same number of crystals as "optimal" has been randomly selected. We may also include "weighted," in which the contribution from each crystal may be weighted by its gross-count SNR.

SourceLocation - currently considering locations 1,7 , and 14.

SourceDepth - any location, but currently 4, 15, 27, 50, and 75\%.

CargoDepth - currently always $100 \%$ - all scenarios include a full cargo container at present. This may be extended in the future.

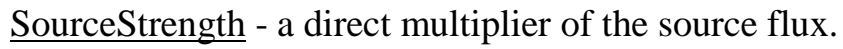

IterationNumber - this number is a placeholder to differentiate statistical realizations of the same scenario.

Each of these .ASC files will be analyzed by all available algorithms, producing a file of filename rawFOM_<algorithm initials>.csv. This file will be written in the format:

$<$ source $>$, <subset $>,<$ src location $>,<$ src depth $>$, < cargo depth $>$, <source strength $>,<$ iteration number $>,<$ alg source FOM $>,<$ alg false alarm FOM $>$

In addition to the variables from the ASC filename, two additional columns are written here, and called "algorithm source FOM" and "algorithm false alarm FOM." These are defined as follows:

Algorithm source FOM - this is the figure of merit returned by the algorithm - in the case of anomaly detectors, this is simply the return value of the algorithm. However, for identification algorithms, this represents a measure of confidence presented to the correct source (e.g., HEU).

Algorithm false alarm FOM - this is the figure of merit expressing confidence in the presence of incorrect sources (e.g., the detection of ${ }^{137} \mathrm{Cs}$ in a scenario that only contains HEU). This should not contain confidences in background or NORM-sources, as these would not be investigated as would regular source alarms. For non-identification schemes, this has no rigorous meaning and should be set to zero. 


\section{Appendix B: Initial Data Analysis}

This appendix presents the initial analysis of the SBRD performance that was incorporated into a presentation in 2010 [10-11]. This provides some of the details of the previous analysis, and how results were obtained, but does not reflect the process used in the this work, since this current work input the data into algorithms and determined estimated detection rate, whereas the previous work scaled the results based on the commercial system results. This appendix does however provide some insight into how the calculations to convolute the MDQ with the cargo distribution are carried out.

A LANL report [8] provided results of testing and analysis of the detection capability of the commercial SBRD systems. The results are fairly similar, and for simplicity, a value of the approximate average of the systems is used. This average, which does not reflect the actual capability of either system, but provides a relatively consistent value is detection of a 1-kg HEU oxide source, in $30 \%$ of a cargo container filled with polyethylene with a density of up to $0.295 \mathrm{~g} / \mathrm{cm}^{3}$.

In order to relate the fraction of cargo volume in which the source can be detected to the maximum depth in cargo at which the source can be detected, we use the following simple model that the contour of MDQ in a cross section of the cargo container is an arc of a circle centered on the top middle of the container. This is true in the limit that all the detectors are located along the middle spine of the top of the cargo container. A diagram of the model is shown in Figure 37.

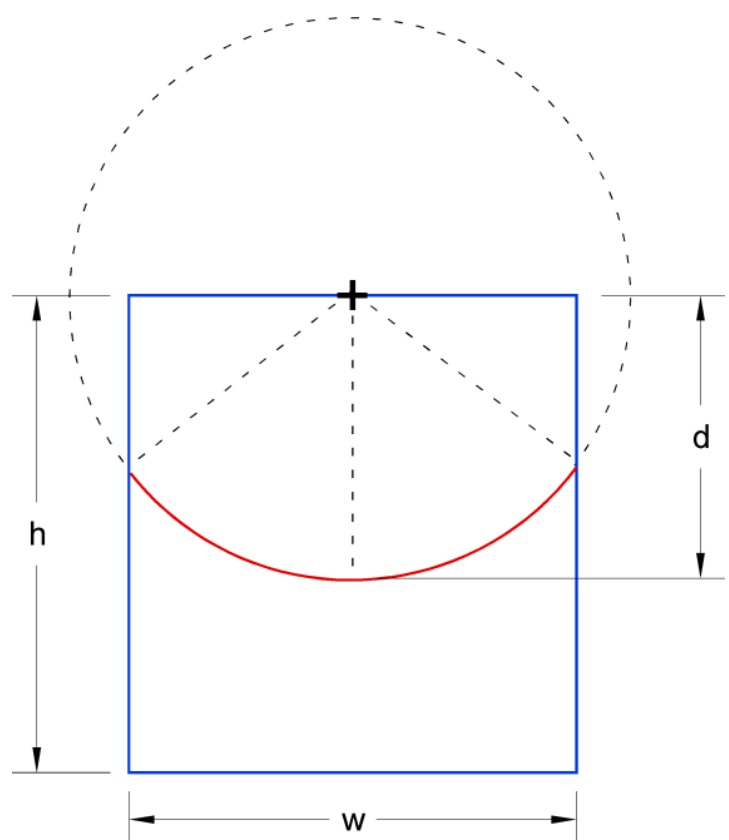

Figure 37. Diagram of the model relating fractional cargo volume to maximum cargo depth for detection.

In this model, the fraction $f$ of the cargo container (in blue) in which the source can be detected (the part above the red line) is related to the depth in cargo container $d$, which is also the radius of the circle, and the height $h$ and width $w$ of the cargo container as 


$$
\begin{array}{cl}
\begin{array}{l}
\text { for } d \quad 0, \\
\text { for } 0<d \quad \frac{w}{2},
\end{array} & \frac{0}{2 h w} \\
f(d, w, h)= & \begin{array}{l}
\text { for } \frac{w}{2}<d \quad h, \\
\text { for } h<d \sqrt{h^{2}+\frac{w^{2}}{4}},
\end{array} \\
& \text { for } \sqrt{h^{2}+\frac{w^{2}}{4}}<d, \quad \frac{d^{2} \sin ^{1} \frac{w}{2 d} \div+\frac{w}{2 d} \div+\frac{w}{2} \sqrt{d^{2} \frac{w^{2}}{4}}}{h w}
\end{array}
$$

In the third line,

$$
d^{2} \sin ^{1} \frac{w}{2 d} \div
$$

is the area of the sector of the circle subtended by the red line and

$$
\frac{w}{2} \sqrt{d^{2} \frac{w^{2}}{4}}
$$

is the area of the two triangles above the sector. In the second line the sector reduces to a half circle and there are no triangles. In the fourth line the final term is the area of the segment of the circle below the container that must be subtracted.

For this calculation we use the inner dimensions of our cargo container model, in which the width $w=$ $2.38 \mathrm{~m}$ and the height $h=2.41 \mathrm{~m}$. This relationship is show in the following plot (Figure 38). Using this relationship, the cargo depth that corresponds to $30 \%$ of the volume fraction is $\sim 1.0 \mathrm{~m}$.

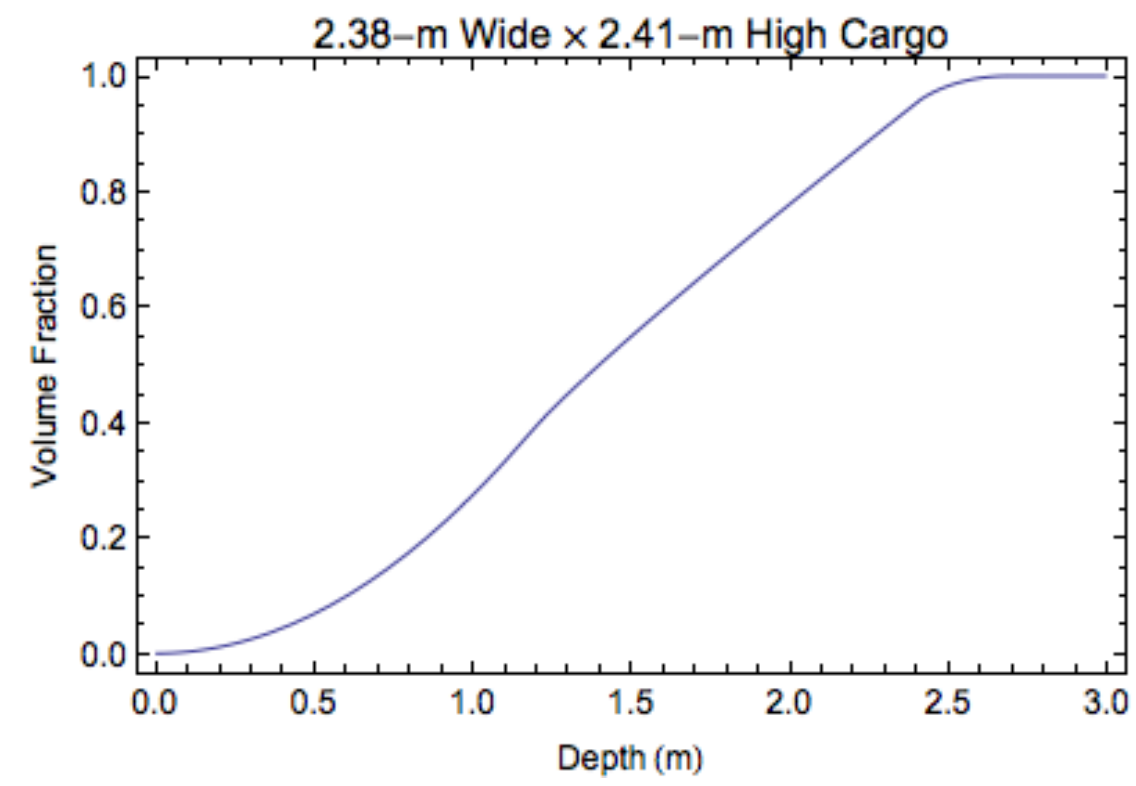

Figure 38. Plot of the fraction of a cargo container in which a source can be detected versus the maximum depth of detection of the source in the cargo container. 
In our modeling the cargo is wood with a density of $0.46 \mathrm{~g} / \mathrm{cm}^{3}$. The attenuation of signal $S / S_{0}$ in the cargo depends on the thickness of the cargo approximately as

$$
S / S_{0} \quad e
$$

where $\mu$ is the attenuation coefficient for the cargo material, $\rho$ is the density of the cargo material, and $t$ is the thickness of cargo traversed. Since $\mu$ is similar for polyethylene and wood, since they polyethylene is made of hydrogen and carbon while wood is mainly hydrogen, oxygen, and carbon, the attenuation in them will be approximately the same if the ratio $\mu \rho$ is the same. Thus we can compensate for the different densities of the two cargos by adjusting the thickness as

$$
t_{\text {wood }}=t_{\text {poly }} \frac{\text { poly }}{\text { wood }} \text {. }
$$

The maximum depth for detecting the LANL source in our wood for the average of the SBRD systems is $0.66 \mathrm{~m}(2.2 \mathrm{ft})$.

To determine the signal we would detect in our model for the LANL source at these depths, we interpolate our calculations of the signal detected in the middle detector from the 5 source positions directly below it for the case of wood cargo filling the container.

Because we need to consider both attenuation in the wood cargo, which is mainly exponential, and attenuation in the air above the cargo, which is mainly a power-law dependence with distance, we consider the detected signal in a window around the main, 186-keV transmission line in HEU.

Interpolating the logarithm of the detected signal versus source depth, as shown in Figure 38 gives a smooth, nearly linear dependence. This is shown in Figure 39 where the points are the results of the MCNP model and the line is an interpolation of the logarithm of rate versus depth.

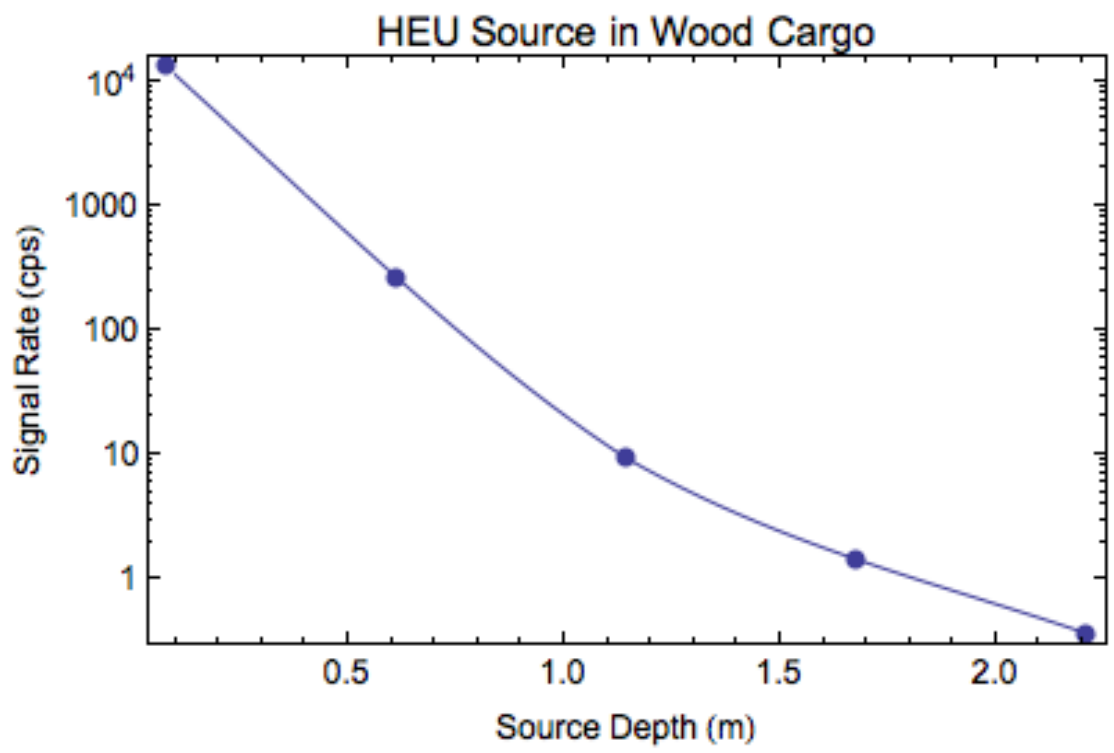

Figure 39. Plot of the signal rate from an HEU source in a load of wood as a function of depth of the source in the wood. The points are the MCNP model and the line is an interpolating function.

Figure 40 shows the inverse of Figure 39; an interpolation function for the source depth versus signal rate. 
PNNL-21948

\section{HEU Source in Wood Cargo}

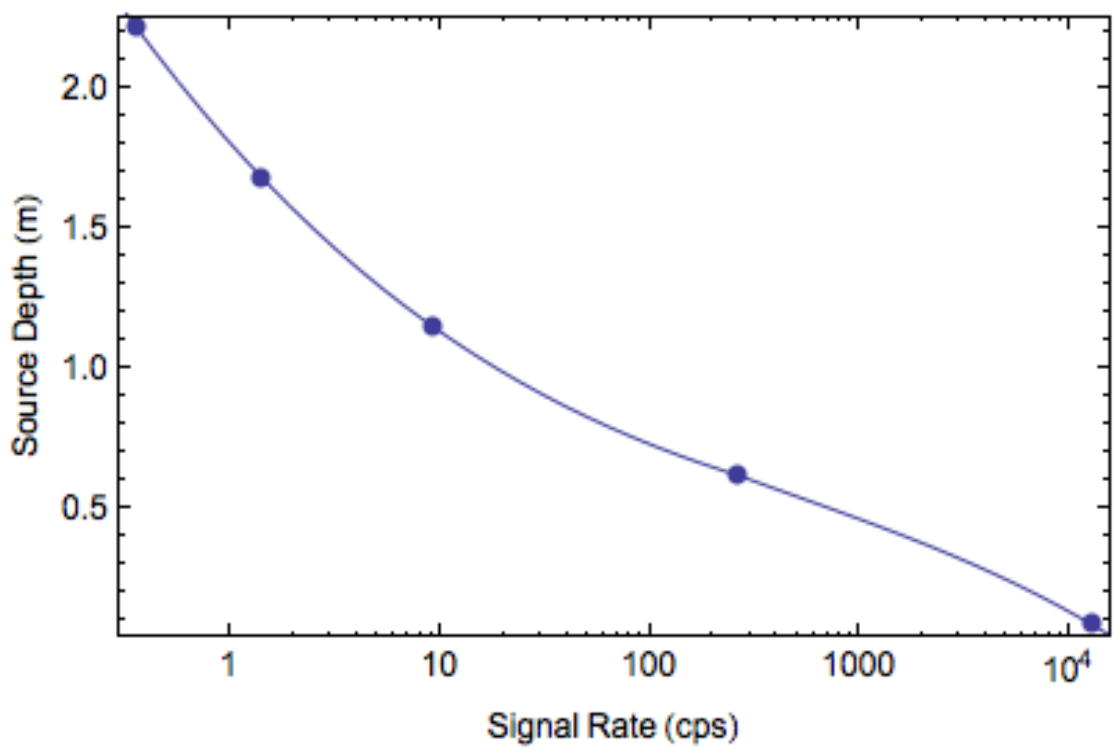

Figure 40. Plot of the depth of an HEU source in wood as a function of the signal rate from the source. The points are the MCNP model and the line is an interpolating function.

Figure 41 shows a similar interpolation for the case of an empty cargo container.

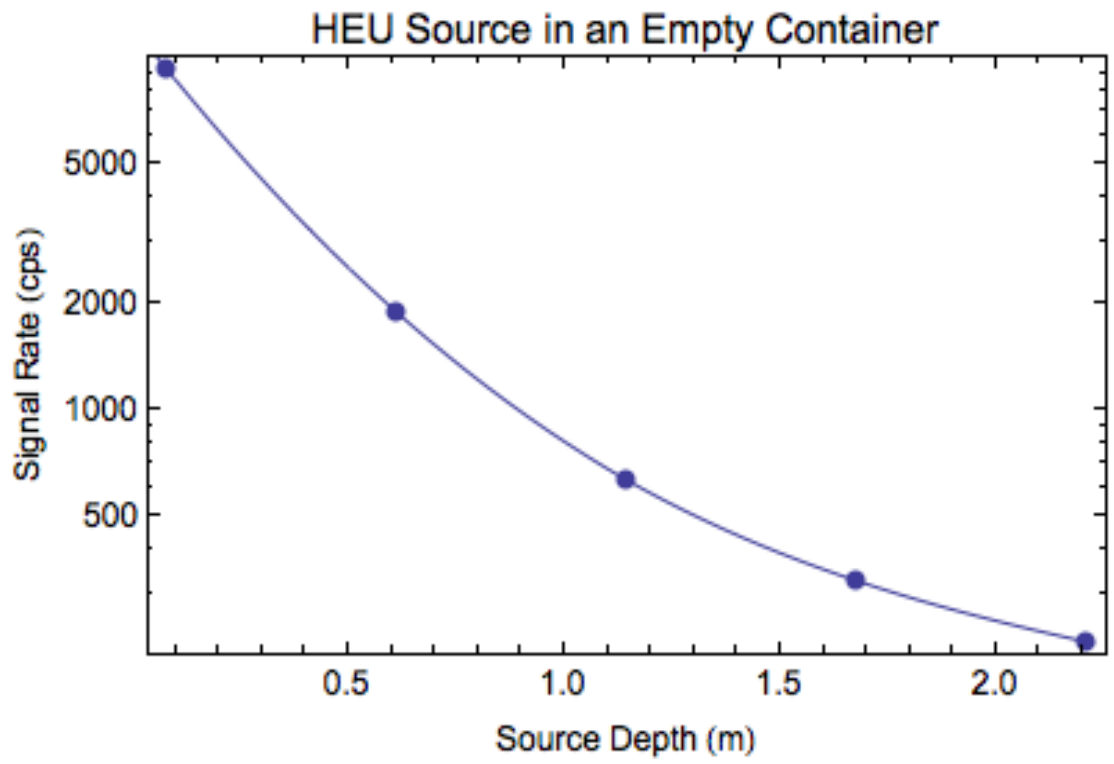

Figure 41. Plot of the signal rate from an HEU source in an empty cargo container as a function of depth of the source in the container. The points are the MCNP model and the line is an interpolating function.

Using the first interpolation above, the signal rate for the middle detector in our model for the LANL source at the maximum detection depth in the energy range from $150 \mathrm{keV}$ to $230 \mathrm{keV}$ is calculated to be $190 \mathrm{cps}$ for the average SBRD.

This depth and signal rate correspond to detection of the LANL source that is approximately equivalent to a 10- $\mathrm{kg}$ sphere of HEU metal, and thus to a MDQ of $10 \mathrm{~kg}$ at this signal rate. We use 
our calculated signal (from a 1-kg cylinder of HEU metal) to scale around this signal to determine the MDQ at other points in the cargo container.

In particular, we scale the MDQ linearly with the signal rate as

$$
\mathrm{MDQ}(\mathrm{r})=\mathrm{MDQ}_{0} \frac{r_{0}}{r}
$$

where $r$ is the signal rate, $r_{0}$ is the calibration signal rate, and $\mathrm{MDQ}_{0}$ is the calibration minimum detectable quantity. This corresponds to assuming that the HEU source consists of individual 1-kg cylinders that are arranged in the cargo container separated by enough distance so that they do not shield each other and so their signals add linearly.

Another possibility would be to assume the HEU source consists of one cylinder. In that case the ratio of the signal of two masses should scale approximately as the area of the cylinder, or approximately as the $2 / 3$ power of the mass of the cylinder. Thus

$$
\frac{r_{1}}{r_{2}} \quad \frac{m_{1}}{m_{2}} \div \text {. }
$$

Inverting gives

$$
\frac{m_{1}}{m_{2}} \quad \frac{r_{1}}{r_{2}} \div \text {, }
$$

so the MDQ scales as the $3 / 2$ power of the detected signal:

$$
\mathrm{MDQ}=\mathrm{MDQ}_{0} \frac{r_{0}}{r} \div \text {. }
$$

Applying the linear scaling to the signal rate in the middle detector for the source positions in the central plane of the cargo container gives the following contour plot of MDQ values, shown in Figure 42. As expected, the $10 \mathrm{~kg}$ LANL source would be detected down to about $0.61 \mathrm{~m}(2 \mathrm{ft})$. 
PNNL-21948

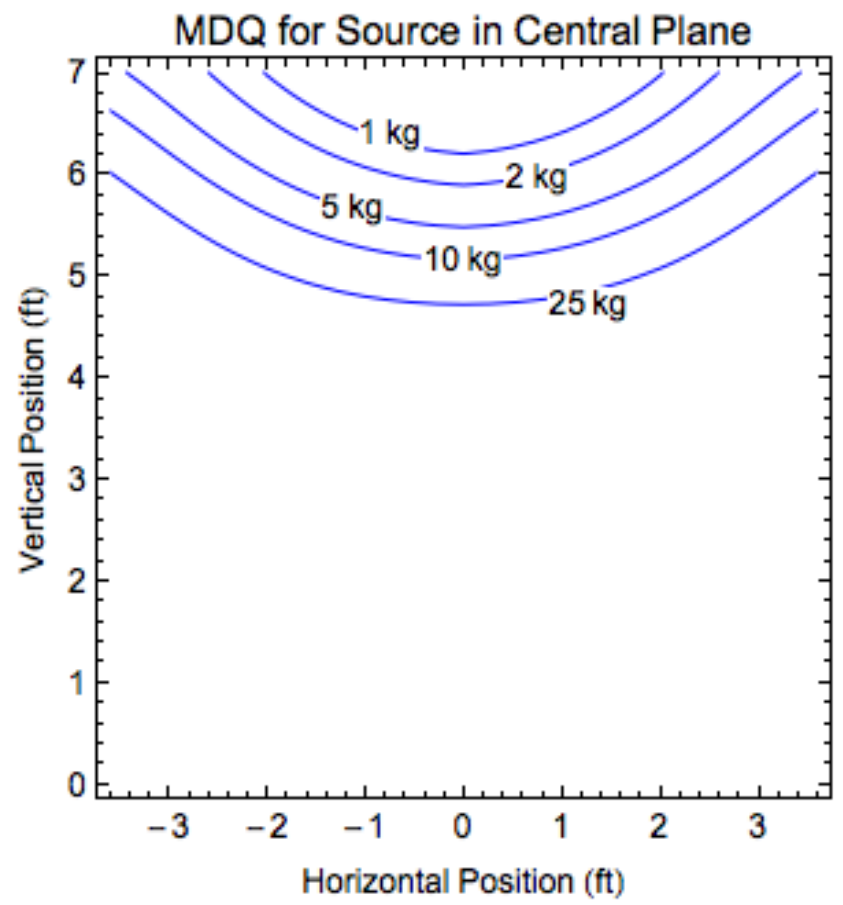

Figure 42. Contour plot of the MDQ for an HEU source in a cargo container full of wood.

The corresponding contour plot for an empty container is shown in Figure 43 . Note that the $1 / r^{2}$ fall off in signal from the top to the bottom of the container is not negligible. As shown in Figure 42 above, it is more than a factor of 30. At the middle on the bottom the HEU source would have to be at least $8 \mathrm{~kg}$ in this particular model to be detected. Note also that the model indicates that there is an enhancement of the detected signal coming from the sides of the container due to the scattering of gamma rays off the container walls. 
PNNL-21948

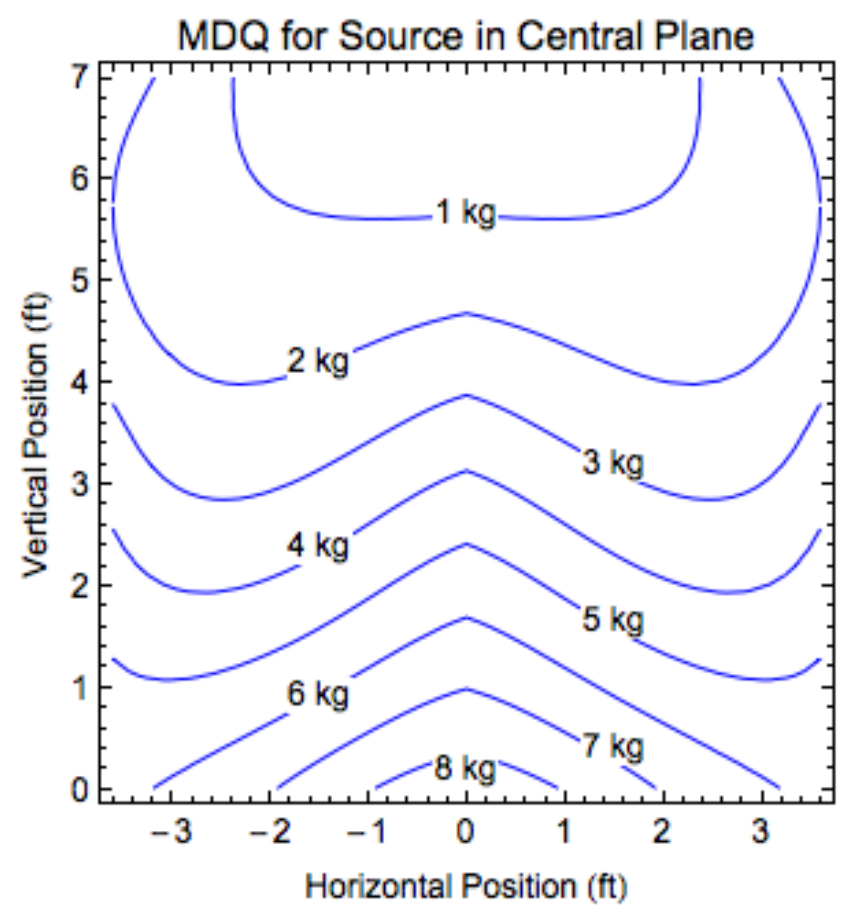

Figure 43. Contour plot of the MDQ for an HEU source in an empty cargo container.

To calculate the volume of cargo scanned for a given source mass, we start with the cargo distribution from a LLNL report [12] of the fraction of TEUs which have a given average cargo density and provide in Figure 44. The data was collected over a 14-day period at a number of ports in the U.S.

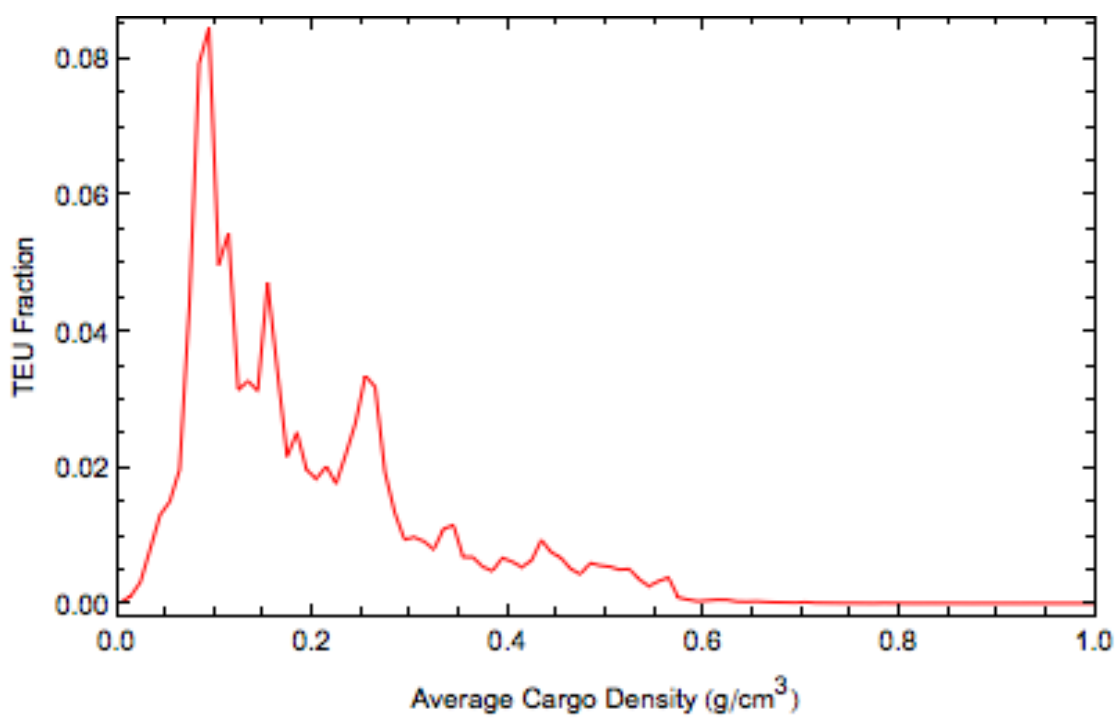

Figure 44. Plot of the fraction of TEUs as a function of average cargo density for cargo containers entering the U.S.

Assuming that this average cargo density is just the cargo weight divided by the volume of a TEU, which is given in the LLNL report as $33.13 \mathrm{~m}^{3}$, the distribution versus cargo weight is given by multiplying by this volume, and the results shown in Figure 45. 
PNNL-21948

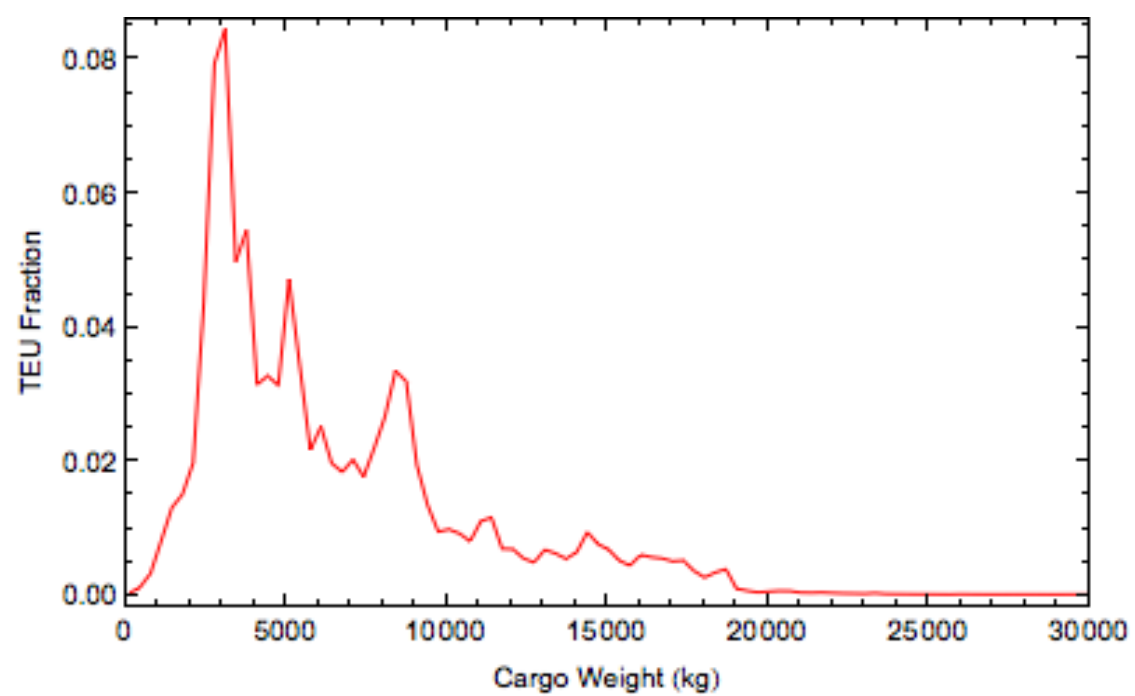

Figure 45. Plot of the fraction of TEUs as a function of cargo weight for cargo containers entering the U.S.

Dividing this cargo weight by the density of wood used in our model gives the distribution versus the volume of wood cargo with the same weight. Dividing by the width and length of our wood cargo in a 20 -ft cargo container gives the thickness of the wood cargo with the same weight. This is shown in Figure 46.

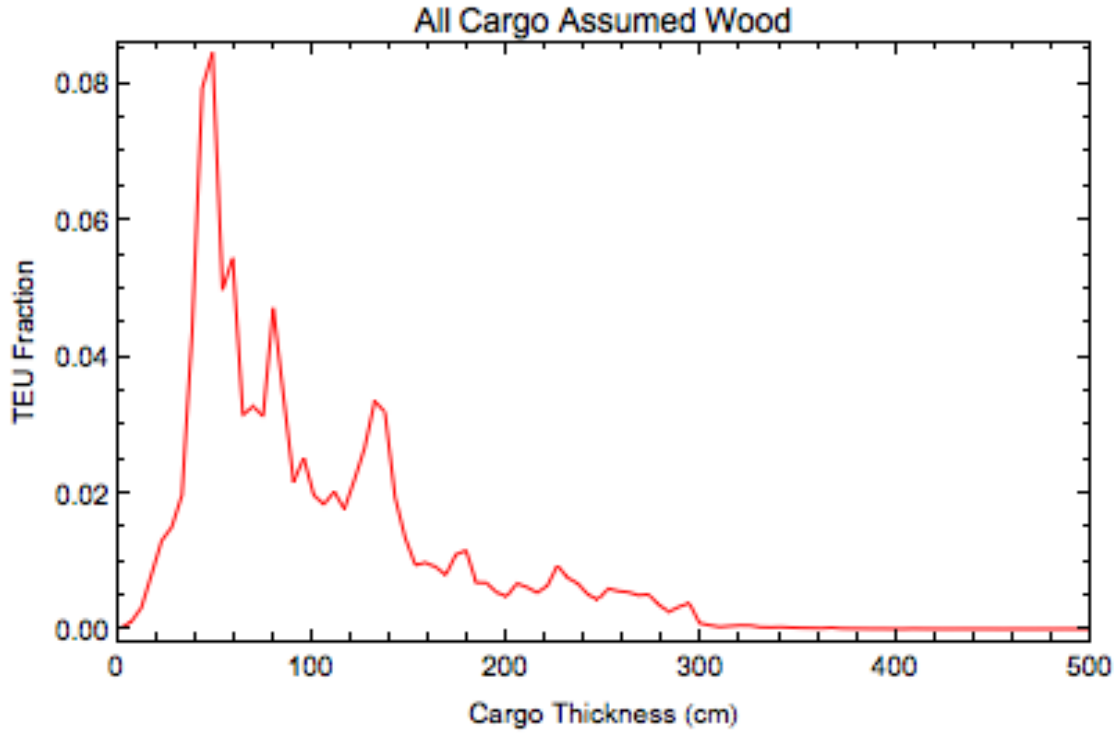

Figure 46. Plot of the fraction of TEUs as a function of the thickness of an equivalent wood cargo for cargo containers entering the U.S.

With this distribution we can calculate the cargo volume fraction that will be scanned as a function of the source mass. For this we need to determine the maximum depth in cargo at which our HEU source can be detected as a function of source mass. Note that this also depends on the thickness of the cargo since the attenuation of signal in the air above the cargo is not negligible and will reduce the depth in cargo at which a given source is detectable.

From above a source is detectable when its detected rate is at least that of the standard source, a $10-\mathrm{kg}$

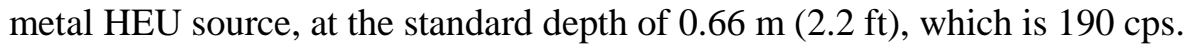




$$
R_{\mathrm{det}} \geq R_{\mathrm{det}}^{0}=190 \mathrm{cps} .
$$

The detected signal rate will be the signal rate emitted by the source and attenuated by the cargo and then attenuated by the air:

$$
R_{\text {det }}=R_{\text {source }} A_{\text {cargo }} A_{\text {air }}
$$

The emission rate of the source of mass $m$ scales with the emission rate of the standard source as

$$
R_{\text {source }}=R_{\text {source }}^{0} \frac{m_{\text {source }}}{m_{0}} \div
$$

where the exponent $a$ is either 1 for linear scaling or $2 / 3$ for scaling with the area of the source. The attenuation in cargo for the source at a depth of $d$ is given by the ratio of the interpolation function for the detected rate above for the depth $d$ to the depth $d=0$.

$$
A_{\text {cargo }}=\frac{R_{\text {cargo }}\left(d_{\text {source }}\right)}{R_{\text {cargo }}(0)}
$$

The attenuation in the air above the cargo is

$$
A_{\text {air }}=\frac{R_{\text {air }}\left(z_{\text {cargo }}\right)}{R_{\text {source }}^{0}}=\frac{R_{\text {air }}\left(h_{\text {det }} t_{\text {cargo }}\right)}{R_{\text {source }}^{0}}
$$

since the distance $z$ from the detector to the top of the cargo is the height $h$ of the detector above the container floor minus the thickness $t$ of the cargo.

Inserting the three terms implies that the detected rate must be

Using the fact that

$$
\begin{aligned}
R_{\text {det }} & =R_{\text {source }}^{0} \frac{m_{\text {source }}}{\mathrm{m}_{0}} \div \frac{R_{\text {cargo }}\left(d_{\text {source }}\right)}{R_{\text {cargo }}(0)} \frac{R_{\text {air }}\left(h_{\text {det }} t_{\text {cargo }}\right)}{R_{\text {source }}^{0}} \\
& =\frac{m_{\text {source }}}{\mathrm{m}_{0}} \div \frac{R_{\text {cargo }}\left(d_{\text {source }}\right)}{R_{\text {cargo }}(0)} R_{\text {air }}\left(\begin{array}{ll}
h_{\text {det }} & \left.t_{\text {cargo }}\right) \\
R_{\text {det }}^{0}
\end{array}\right.
\end{aligned}
$$

gives

$$
R_{\text {cargo }}(0)=R_{\text {air }}\left(h_{\text {det }} t_{\text {cargo }}\right)
$$

$$
R_{\text {det }}=R_{\text {cargo }}\left(d_{\text {source }}\right) \frac{R_{\text {air }}\left(h_{\text {det }} t_{\text {cargo }}\right)}{R_{\text {air }}\left(h_{\text {det }} t_{0}\right)} \frac{m_{\text {source }}}{\mathrm{m}_{0}} \div \quad R_{\text {det }}^{0} .
$$

Rearranging gives

$$
R_{\text {cargo }}\left(d_{\text {source }}\right) \quad R_{\text {det }}^{0} \frac{R_{\text {air }}\left(h_{\text {det }} t_{0}\right)}{R_{\text {air }}\left(h_{\text {det }} t_{\text {cargo }}\right)} \frac{\mathrm{m}_{0}}{m_{\text {source }}} \div \text {. }
$$

This equation is solved for $d$ using the interpolation function in Figure 40 above that is just the inverse $d(R)$ of $R(\mathrm{~d})$

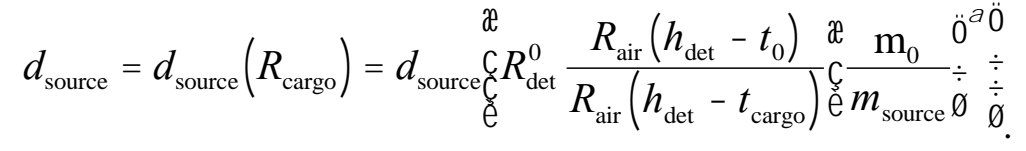

For a given source mass and cargo thickness we divide that depth by the cargo thickness to determine in what fraction of the cargo volume that source could be detected, ignoring any air volume above the cargo. We multiply that by the TEU fraction for that cargo thickness. We do this for all cargo thickness channels and then add up to get the total fraction of the cargo volume in which a source of 
PNNL-21948

the chosen mass could be detected. This is plotted in Figure 47 for various source masses in a 40 -foot cargo container.

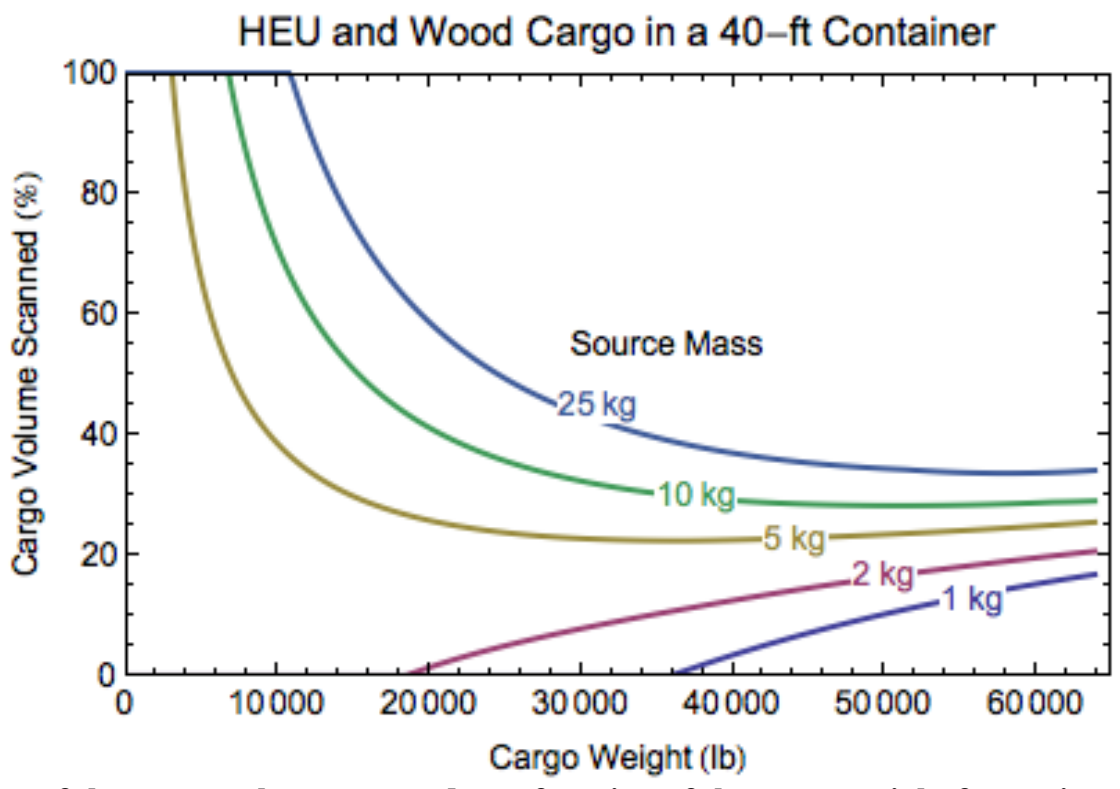

Figure 47. Plot of the cargo volume scanned as a function of the cargo weight for various HEU source masses.

Summing over all cargo weights at each source mass with the LLNL distribution of cargo fraction versus cargo weight, or equivalently cargo thickness, gives the distribution of cargo volume scanned as a function of the HEU source mass and is shown in Figure 48.

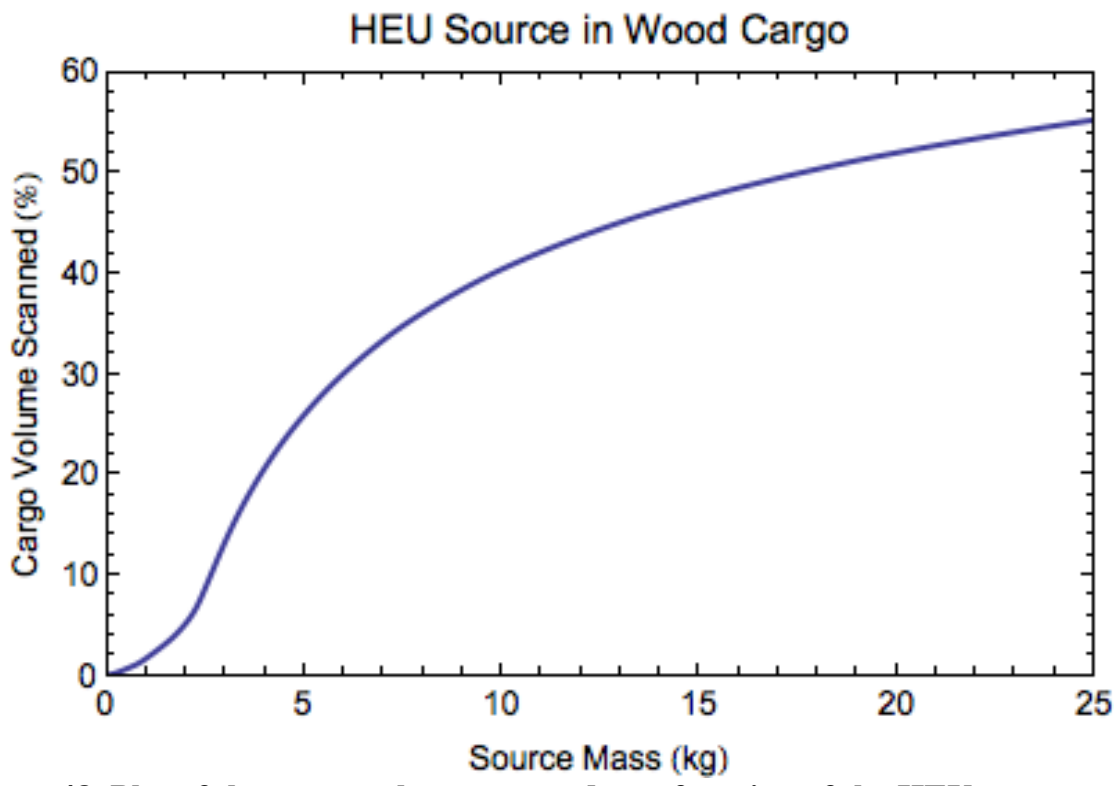

Figure 48. Plot of the cargo volume scanned as a function of the HEU source mass.

As mentioned above, this analysis has been done with the assumption that the signal from the HEU source scales linearly with the mass of the source. Figure 49 compares the result shown in Figure 48 to the same analysis done with the assumption of a 2/3-power-law scaling of the signal to the source 
mass. As can be seen, the final result is not very sensitive to the form of the dependence of signal on source mass.

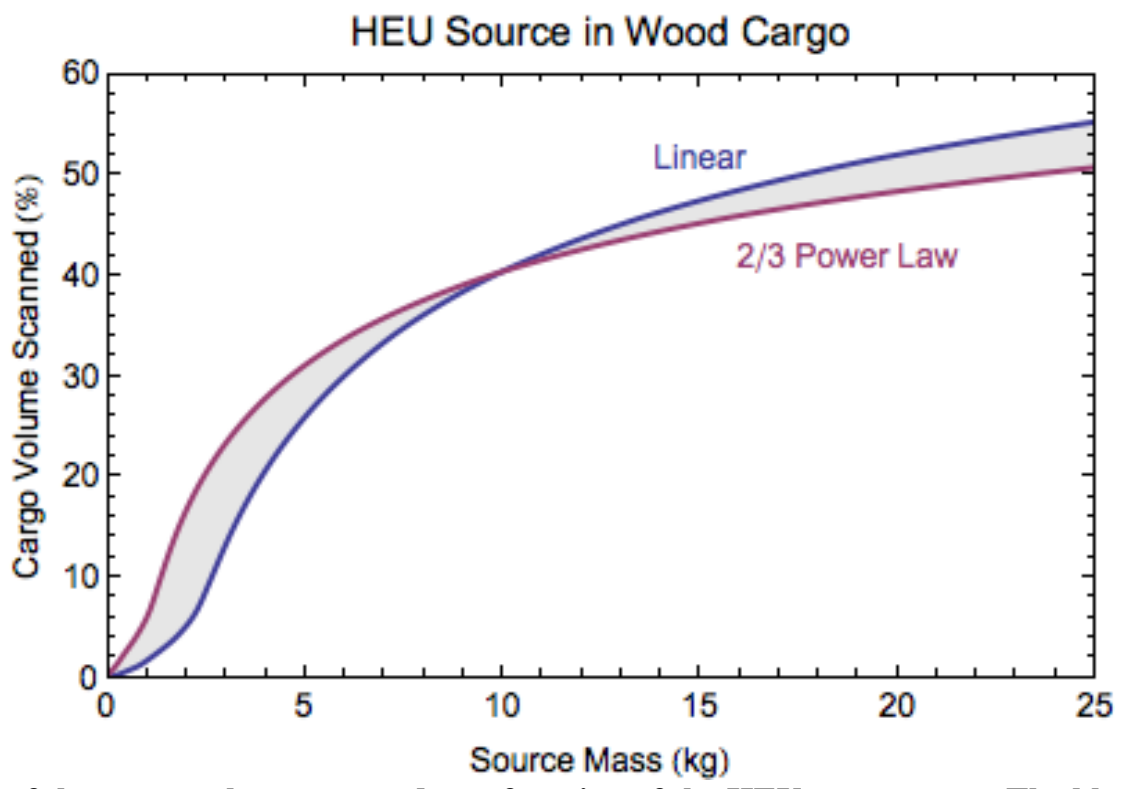

Figure 49. Plot of the cargo volume scanned as a function of the HEU source mass. The blue curve depicts a linear dependence of signal on source mass while the red curve a $2 / 3$ power-law dependence.

For the Enhanced SBRD (2010), we developed an SBRD model with thirty-three 4 in $\times 4$ in $\times 16$ in $\mathrm{NaI}(\mathrm{Tl})$ detectors arranged along the length of the spreader bar, which is 2-3 times as much detector material as the commercial systems had in 2008.

Several of the limitations of the commercial systems (treated as a whole) in 2008 were limited detector area, limited summing of all detectors, and integration of the signal over the entire move. Analysis of our enhanced model shows that there would be an enhancement factor of the signal-tonoise ratio by a factor of 2.1 by increasing the detector material, and applying optimized detector summing and integration over time. This increases the depth of detection of the LANL 10-kg HEU source by $0.11 \mathrm{~m}(0.35 \mathrm{ft})$ from $0.66 \mathrm{~m}(2.2 \mathrm{ft})$ to $0.77 \mathrm{~m}(2.5 \mathrm{ft})$.

In addition, observations during the Tacoma measurements [6-7] suggested that there is a possible additive enhancement term due to using enhanced algorithms that will allow the system to detect 0.35 $\mathrm{m}(0.8 \mathrm{ft})$ deeper into the wood cargo. This further increases the depth of detection for the LANL source to $1.01 \mathrm{~m}(3.31 \mathrm{ft})$. This last additive enhancement would correspond to a multiplicative improvement in the signal-to-noise ratio by a factor of 4.6 on top of the factor of 2.1 above. This increase in the depth of detection in the Enhanced SBRD (2010) is shown in Figure 50, where it is superimposed on the interpolation of rate versus source depth from Figure 39. 
PNNL-21948

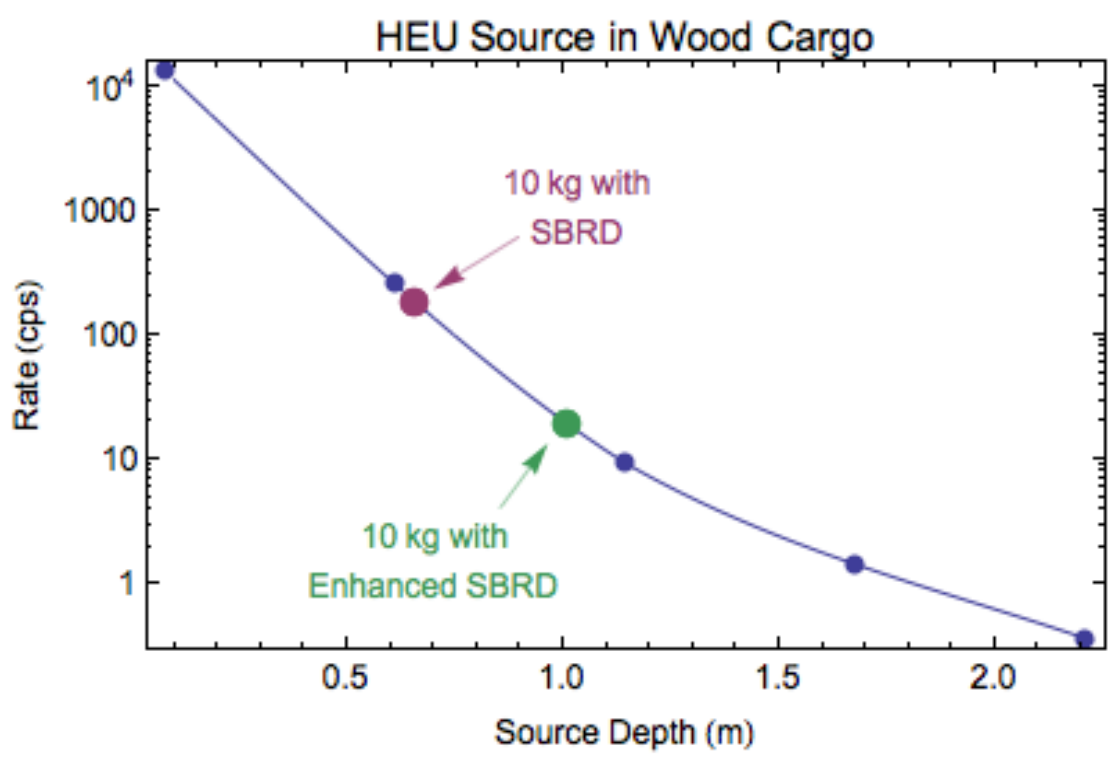

Figure 50. Plot of the signal rate from an HEU source in a load of wood as a function of depth of the source in the wood. Points indicating the maximum depth for detection of a 10-kg source with the SBRD and with the Enhanced SBRD are shown.

Repeating the analysis above with this new value of the detected rate $R_{\text {det }}^{0}$ for the standard, $10-\mathrm{kg}$ source with the Enhanced SBRD (2010) produces the dependence of total detectable cargo volume scanned as a function of the MDQ and is shown in Figure 51. Also shown are the distributions for the average of the commercial SBRD.

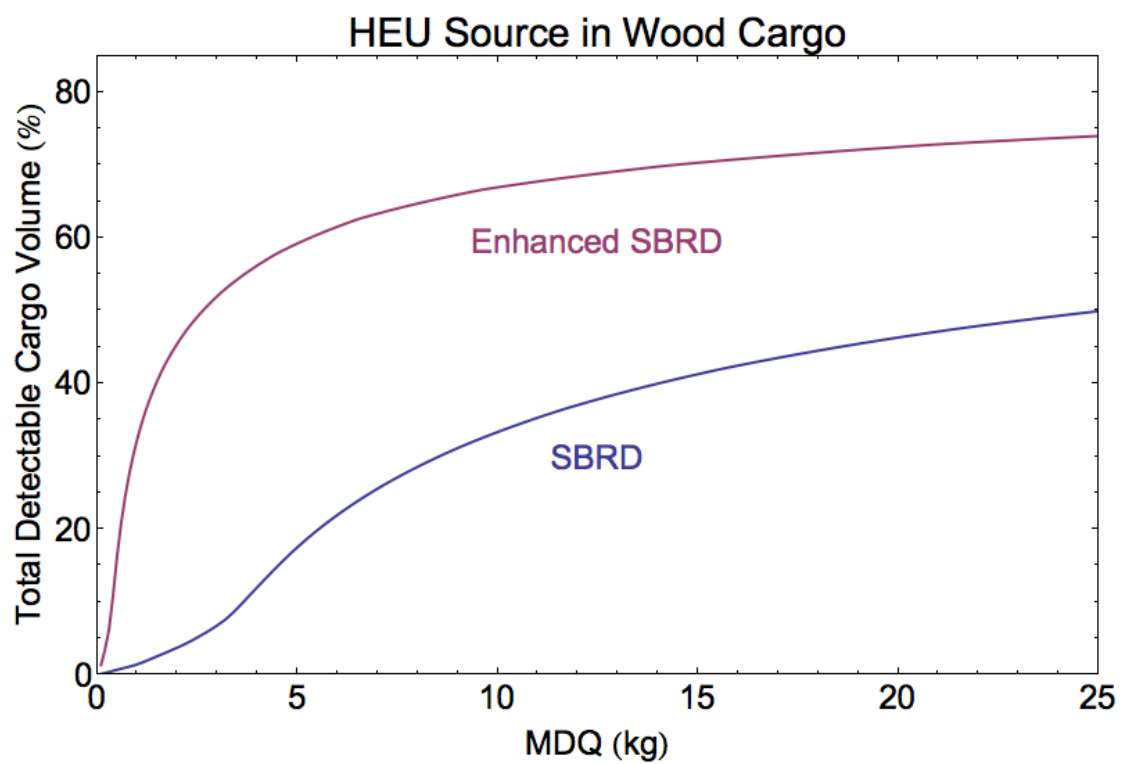

Figure 51. Plot of the total detectable cargo volume scanned as a function of the MDQ of HEU for the case that the source is anywhere in the cargo.

A similar set of calculations made with the HEU source always sitting on the bottom of the container gives the worst-case scenario for a spreader-bar-based detection system. This is shown in Figure 52. 
PNNL-21948

HEU Source in Wood Cargo - Worst Case

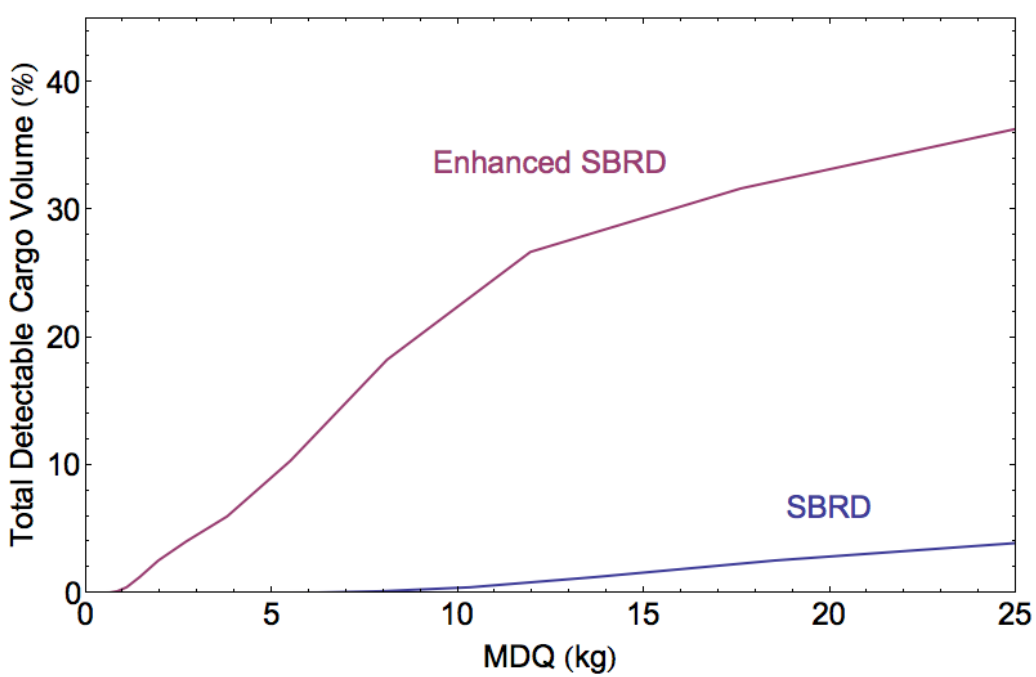

Figure 52. Plot of the total detectable cargo volume scanned as a function of the MDQ of HEU for the case that the source is always on the bottom of the cargo container as a worst case. 


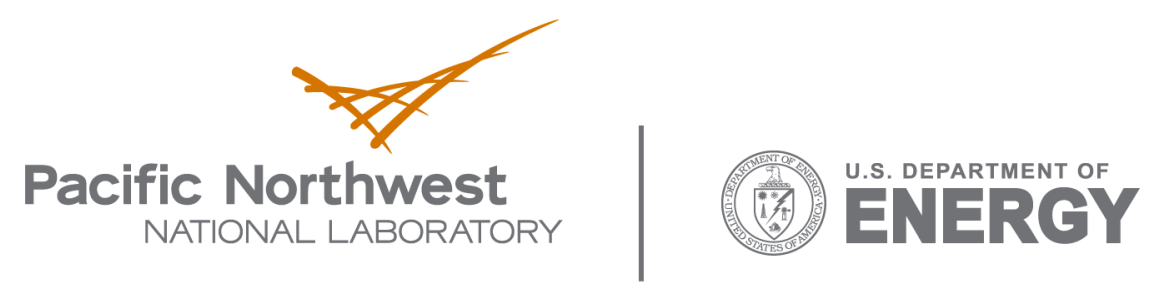

902 Battelle Boulevard

P.O. Box 999

Richland, WA 99352

1-888-375-PNNL (7665)

www.pnl.gov 